\title{
DESIGN AND EVALUATION
}

\section{OF SYSTEM CONFIGURATIONS FOR AN}

\section{EUV MASK INSPECTION MICROSCOPE}

EUV AlM tool design study

\author{
Report 3 for \\ AIM Design Study \\ Anton Barty and John S. Taylor \\ Lawrence Livermore National Laboratory \\ L-395, PO Box 808, Livermore, CA 94550 \\ (barty1@1lnl.gov, jstaylor@1lnl.gov) \\ Russell Hudyma \\ Paragon Optics Inc. \\ 218 East Ridge Drive \\ San Ramon, CA, 94583 \\ (rhudyma@attbi.com) \\ Eberhard Spiller \\ Spiller X-ray optics \\ 381 Marie Common \\ Livermore, CA, 94550 \\ (spiller1@llnl.gov, espill@attglobal.net)
}

20 August 2002

Submitted to:

Jan-Peter Urbach

International SEMATECH

2706 Montopolis Drive

Austin, TX 78741 


\section{DISCLAIMER}

This document was prepared as an account of work sponsored by an agency of the United States Government. Neither the United States Government nor the University of California nor any of their employees, makes any warranty, express or implied, or assumes any legal liability or responsibility for the accuracy, completeness, or usefulness of any information, apparatus, product, or process disclosed, or represents that its use would not infringe privately owned rights. Reference herein to any specific commercial product, process, or service by trade name, trademark, manufacturer, or otherwise, does not necessarily constitute or imply its endorsement, recommendation, or favoring by the United States Government or the University of California. The views and opinions of authors expressed herein do not necessarily state or reflect those of the United States Government or the University of California, and shall not be used for advertising or product endorsement purposes.

This work was performed under the auspices of the U. S. Department of Energy by the University of California, Lawrence Livermore National Laboratory under Contract No. W-7405-Eng-48.

This report has been reproduced directly from the best available copy.

Available electronically at http://www.doe.gov/bridge

Available for a processing fee to U.S. Department of Energy

and its contractors in paper from

U.S. Department of Energy

Office of Scientific and Technical Information

P.O. Box 62

Oak Ridge, TN 37831-0062

Telephone: (865) 576-8401

Facsimile: (865) 576-5728

E-mail: reports@adonis.osti.gov

Available for the sale to the public from

U.S. Department of Commerce

National Technical Information Service

5285 Port Royal Road

Springfield, VA 22161

Telephone: (800) 553-6847

Facsimile: (703) 605-6900

E-mail: orders@ntis.fedworld.gov

Online ordering: http://www.ntis.gov/ordering.htm

OR

Lawrence Livermore National Laboratory

Technical Information Department's Digital Library 
http://www.llnl.gov/tid/Library.html 


\section{CONTENTS}

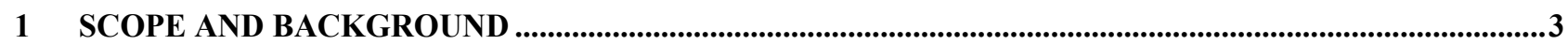

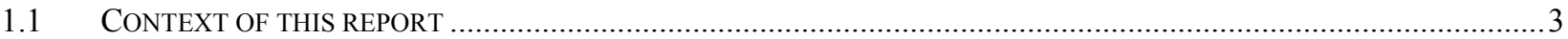

1.2 SYSTEM REQUIREMENTS FOR AIM AND HIGH-RESOLUTION MODES ...........................................................

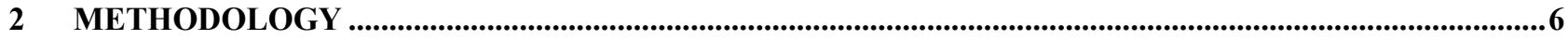

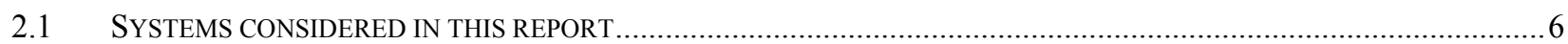

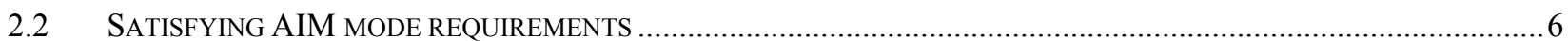

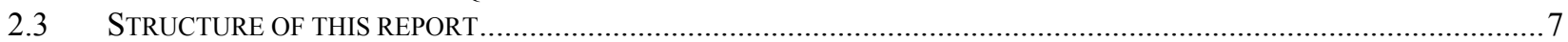

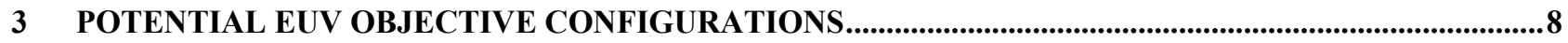

3.1 METRICS OF OPTICAL SYSTEM PERFORMANCE: MTF, DISTORTION AND THROUGH FOCUS BEHAVIOUR ...............8

3.2 TYPICAL PERFORMANCE OF A 6-MIRROR 0.25NA SYSTEM..........................................................................

3.3 PERFORMANCE AND FIELD OF VIEW REQUIREMENTS FOR AIM MODE ........................................................... 11

EXISTING OBJECTIVE DESIGNS FOR AIM MODE ……............................................................... 14

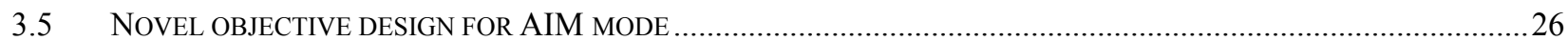

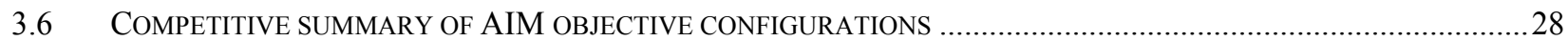

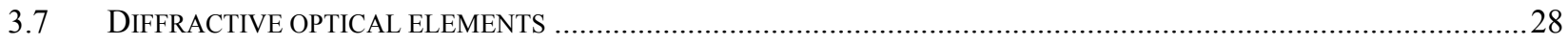

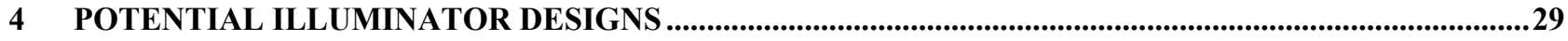

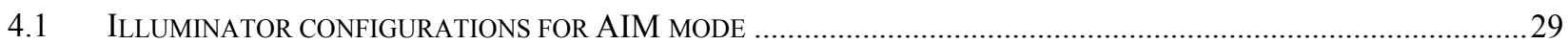

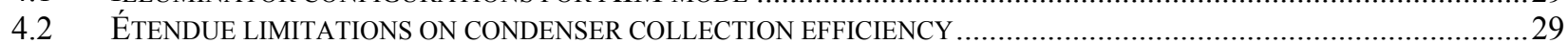

4.3 ILLUMINATION AND OBJECTIVE CONFIGURATIONS FOR HIGH-RESOLUTION MODE...............................................30

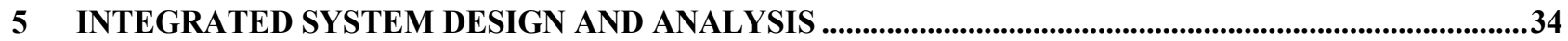

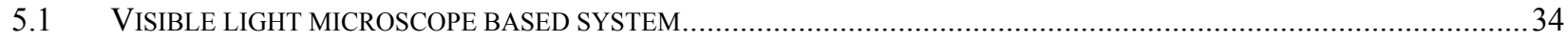

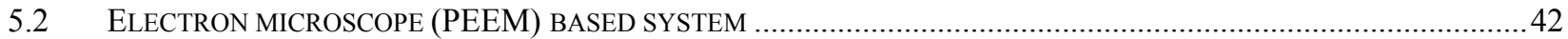

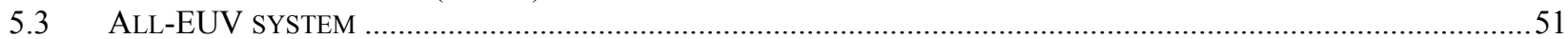

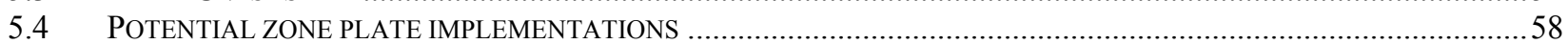

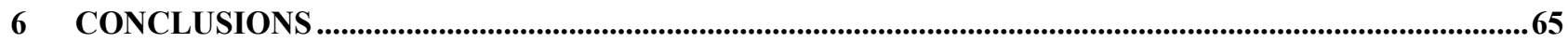

This work was performed under the auspices of the U.S. Department of Energy by University of California Lawrence Livermore National Laboratory under contract No. W-7405-Eng-48. The authors acknowledge funding from International SEMATECH under Project Lith 341. UCRL-CR-149774. 


\section{Scope and background}

\subsection{Context of this report}

This document is the third sub-report of the EUV AIM design study being conducted at LLNL on behalf of International Sematech (ISMT). The purpose of this study as identified in section 1.2 of the statement of work is to research the basic user requirements of an actinic defect characterisation tool, potential design configurations and toplevel specifications. The objectives of this design study specifically identified in section 1.3 of the statement of work were to

1. Determine the user requirements of an actinic defect characterisation tool

2. Determine if an EUV AIM tool is an appropriate platform for actinic defect characterisation

3. Determine possible design configurations and top-level performance specifications

4. Identify potential technical issues and risks of different technical approaches

5. Provide estimates of cost relating to different technical approaches; and

6. Provide simulated performance for key subsystems and the entire system

The sub-sections of the study to be addressed were accordingly defined in the statement of work as being:

1. Formulation of top-level specifications

2. Identification of system configurations suitable for meeting the top-level specifications

3. Preliminary design of imaging systems

4. Preliminary design of illumination systems

5. Prediction and comparison of performance through aerial image calculation

6. Identification of sub-system requirements

7. Identification of potential vendors

8. Estimation of system cost

9. Identification of technical issues

10. Definition of technology transfer or development required

Points 1 and 2 have already been addressed in previous reports to ISMT. This document addresses points 3 to 7 , and 9 to 10 of the above list - formulation of a preliminary design of the imaging and illumination systems and the evaluation and comparison of potential designs through aerial image analysis. As such this report should be read in conjunction with and in the context of the previous two reports which separately addressed points 1 and 2 above $e^{1}$. Item 8 , estimation of system cost, will be addressed in a separate confidential report to ISMT.

Following the top level specifications and down-selection document we identify the following modes of operation of an EUV AIM tool:

\section{Aerial Image Microscope (AIM) mode}

In this mode the microscope is designed to emulate the illumination and imaging characteristics of a stepper to enable rapid evaluation of mask defects without the need for a resist exposure step. The main uses of the microscope in this mode would be:

- Review of multilayer and pattern defects to determine their printability

- Defect review following a repair process to assess the success of the operation

- Investigation of the effects of illumination and NA on the printed image

- Process window analysis of defects and other mask features

- Characterising defects on both patterned and unpatterned masks (i.e. blanks)

An essential characteristic of operation in this mode is that the illumination and imaging conditions should emulate that of a production stepper system.

\section{High-resolution mode}

This imaging mode is designed to obtain the highest possible resolution on the mask for understanding and characterising defects. Potential uses for this imaging mode include:

\footnotetext{
${ }^{1}$ Note that certain aspects of the material covered in this report have also been presented at the ISMT EUV AIM tool bidders' conferences held at Altis, Corbeil-Essonnes, France and Lawrence Berkeley Laboratories, Berkeley, CA.
} 
- Gaining sufficient information to associate defects with particular processes, such as multilayer deposition or patterning

- Assessing the size, reflectivity and phase impact of surface and multilayer anomalies

- High-resolution data for repair of features that do not clearly appear in AIM mode (eg: OPC features)

In this mode it is not essential that the illumination and imaging conditions match that of a production stepper system as the goal is to obtain high-resolution data on the mask defects for the purpose of, amongst other things, determining a suitable repair strategy.

The suitability of various system designs for statisfying these requirements are considered below. The systems considered in this report are those identified in the initial down-selection as potential candidates capable of satisfying the top level specifications for an EUV AIM tool.

\subsection{System requirements for AIM and high-resolution modes}

The key specifications for an EUV AIM tool operating in both AIM and high-resolution modes were identified in the previous LLNL reports and are summarised below. These specifications are substantially identical to those identified in the initial down-selection report except for the requirement of $0.075 \mathrm{NA}$ being relaxed to $0.0625 \mathrm{NA}$. This followed further investigation of the patent literature which indicated that the specifications for a first-generation production tool were likely to be $0.25 \mathrm{NA}$ at $4 \mathrm{x}$ reduction rather than $0.3 \mathrm{NA}$ at $4 \mathrm{x}$ reduction. The key specifications for both AIM and high-resolution modes, discussed in a previous report delivered to ISMT, are identified in Table 1 below.

\begin{tabular}{|c|c|c|}
\hline & AIM mode & High-resolution mode \\
\hline Wavelength & $13.5 \mathrm{~nm}$ & $13.5 \mathrm{~nm}\left(\mathrm{TBD}^{2}\right)$ \\
\hline Bandwidth & $2 \%$ FWHM & TBD \\
\hline NA & $0.0625 \mathrm{NA}$ & $0.2-0.45 \mathrm{NA}$ \\
\hline Resolution limit & $\delta=107 \mathrm{~nm}$ & $\delta=15 \mathrm{~nm}($ at $0.45 \mathrm{NA})$ \\
\hline Pixel size at mask ${ }^{3}$ & $\Delta x=26 n m$ & $\Delta x \leq 7.5 \mathrm{~nm}$ \\
\hline Field of view ${ }^{4}$ & $50 \times 50 \mu \mathrm{m}$ & TBD \\
\hline Angle of incidence & $6^{\circ}$ & TBD \\
\hline Physical size ${ }^{5}$ & $\begin{array}{l}\text { Optics package }<2 \mathrm{~m} \text { long } \\
\text { Preferably }<1 \mathrm{~m} \text { long }\end{array}$ & Same \\
\hline Throughput & $<2$ s per exposure & Same \\
\hline Source & $\begin{array}{l}\text { Compatible with a } \\
\text { compact source }\end{array}$ & Same \\
\hline
\end{tabular}

Table 1

Key specifications and requirements for an EUV AIM tool in AIM and both high-resolution modes.

\subsubsection{AIM mode requirements}

For AIM mode, where the tool is used to provide reliable data for defect printability analysis, it is essential that the results obtained by measuring the aerial image with an AIM tool provide the same information that would be obtained in a stepper system. Mimicking the performance of a stepper, albeit over a smaller field, is therefore essential if the results obtained using an AIM tool are to provide a reliable assessment of defect printability in a production tool.

\footnotetext{
${ }^{2}$ The actual wavelength and bandwidth for high-resolution mode will depend on angles of incidence at the mask.

${ }^{3}$ A factor of 2 oversampling has been included in this figure.

${ }^{4}$ This is a suggested value. The actual field of view and size of the field over which imaging should be corrected to AIM quality imaging is open to determination between customer and vendor.

${ }^{5}$ The main requirement is that the unit fit readily into existing mask shop environment. In this regard it is the size of the overall optics package that is important - the total system path length may be much greater due to multiple reflections within the optical system.
} 
Production stepper optics strive to achieve diffraction-limited performance across the field, thus for an AIM tool we also desire a system capable of mimicking this diffraction limited imaging - albeit producing a magnified rather than reduced image. Probing through-focus behaviour of defects for process window analysis is one of the main applications for AIM mode, thus it is also essential that the through-focus behaviour of the AIM tool mimic that of a typical stepper system.

Demonstrated equivalence of the through-focus aerial image performance of an AIM system with that of a production system is essential for full qualification of different technical approaches to implementing AIM mode imaging. For full qualification of a particular design it would be necessary to include the effects of residual manufacturing aberrations and flare when evaluating projected AIM tool performance. Manufacturing aberrations affect both in-focus and through-focus behaviour, and therefore impact on the aerial image. Flare directly affects contrast, thus emulating the flare characteristics of a stepper system also needs to be considered when judging the equivalence of aerial image performance ${ }^{6}$.

\subsubsection{High-resolution mode requirements}

The design options for high-resolution mode is more analogous to traditional microscope design where the goal is to maximise spatial resolution. It is not essential to emulate the through-focus imaging performance of a stepper system thus there is greater flexibility in system design and a greater range of potential values for parameters such as angle of incidence, NA and wavelength. The specifications on these quantities are therefore open to variation but are anticipated to be in the range of values described in the table above.

\footnotetext{
${ }^{6}$ In this regard it is important to note that it may be necessary to illuminate a larger area than the imaged field both so that the flare is uniform across the imaged field and so that flare from regions outside of the imaged field can be properly captured in the aerial image. It is possible to emulate the effect of flare in software by convolving with a flare point spread function provided the flare of the AIM tool is less than that of a production stepper system.
} 


\section{Methodology}

\subsection{Systems considered in this report}

A range of candidate systems for satisfying AIM mode requirements were discussed in the initial down-selection document, and the following configurations were selected for further investigation within the scope of this design study:

1. AIM objective with EUV secondary magnification,

2. AIM objective with electron optics secondary magnification,

3. AIM objective with visible light secondary magnification.

All of the systems require an EUV objective and it is likely that the same objective lens configurations may be suitable for use with many or all of the secondary magnification stages, thus the design of a set of objective lenses is required regardless of whether one or more secondary magnification stages are selected. Accordingly in this report we first investigate objective configurations suitable for AIM mode operation and then proceed to integrate those objective designs into the three potential system configurations identified for further investigation. Options for integrating a highresolution mode either through modification of the AIM mode objective or through use of a dedicated, interchangeable high-resolution objective are also considered.

The abovementioned systems are in principle capable of meeting the top-level requirements of an EUV AIMS tool, as discussed in the initial down-selection report. The fact that we have down-selected to these options does not preclude the existence of other solutions; rather down-selection represents a necessary narrowing of scope of investigation for the purposes of demonstrating the feasibility of designing an AIM tool and provides a forum for raising technical issues concerning different approaches. It should be recognised by both vendors and potential customers that alternative solutions may provide viable alternative implementations for AIM mode imaging even though they have not been pursued to the point of a preliminary design in this study, and that notwithstanding the necessary process of down-selection the analysis provided in this report should serve as a guide for the level of analysis required in evaluating the capacity of alternative implementations to satisfy the requirements of AIM mode imaging.

\subsection{Satisfying AIM mode requirements}

As discussed in section 1.2, demonstrated equivalence of the through-focus aerial image performance of an AIM system with that of a production stepper system is essential for full qualification of technical approaches to implementing AIM mode imaging. Whilst through-focus aerial image simulation across the AIM field is required for full design qualification, we adopt the following approach to evaluating and down-selecting different optical system designs:

1. The design must be of a practical size and satisfy the requirement of $0.0625 \mathrm{NA}$ clear aperture at $6^{\circ}$ angle of incidence using $13.5 \mathrm{~nm}$ EUV light with a bandwidth of $2 \%$, as described in the table in section 1.2. If a design departs from these requirements the manner in which it does so is clearly identified and the consequences of doing so discussed.

2. A preliminary system characterisation is performed by comparing the incoherent modulation transfer function (MTF), through-focus behaviour and distortion of the optical system across the field. These must be shown to not degrade the optical performance in order for the optical system to faithfully emulate the imaging performance of a stepper system. We perform this analysis across the field of view quoted for AIM mode imaging in order to demonstrate how the measurement will be affected by where the object is located in the field of view ${ }^{7}$.

3. Once the requirements of (1) and (2) are satisfied, aerial images are calculated across the field for a characteristic feature size to demonstrate the equivalence of aerial images measured using the AIM objective with the performance of a stepper system. For the purposes of this study we choose to calculate the aerial image at $6250 \mathrm{lp} / \mathrm{mm}$, corresponding to $80 \mathrm{~nm}$ dense features on the mask or $20 \mathrm{~nm}$ dense features

\footnotetext{
${ }^{7}$ Depending on design the field of view for AIM mode imaging may be less than the field of view of the whole system. For example, an objective may have a visible field of view of 50x50 $\mu \mathrm{m}$ which can be used for navigating around the mask, whilst AIM quality imaging is restricted to a $20 \times 20 \mu \mathrm{m}$ sub-field in the centre of the field of view. In this case the field of view useable for AIM mode must be clearly identified and equivalence of AIM mode imaging with stepper performance demonstrated over the entire AIM field. Discussion of the performance expected over the whole field of view would also be useful so that the amount of image degradation at the edge of field can be assessed.
} 
on the wafer. As discussed later this is close to the resolution limit of a $0.25 \mathrm{NA}, 6$-mirror camera, thus performance at this feature size provides a good threshold estimate of aerial image performance of a particular design.

4. Full system qualification requires comparing 2D aerial image simulations of different types of defects at both the best and worst field points within the AIM mode area and demonstrating equivalence with the aerial images anticipated for a stepper system. This should be done for both perfect patterns of critical CD dimensions as well as masks with amplitude and phase defects, and should take into account the effects of flare, design aberrations and anticipated residual aberrations from manufacturing and alignment. Manufacturing aberrations affect both in-focus and through-focus behaviour, and therefore impact on aerial image assessment. Flare directly affects contrast and, thus, the image slope used for printability analysis, and it is therefore important that some capacity be included for either matching the flare of a stepper system in hardware or simulating the effect of flare by convolution of the image in software with a flare point spread function ${ }^{8}$. The important point is that conclusions about defect printability must be the same in an AIM tool as in a stepper system, and should be consistent across the field of view quoted for AIM mode (which may or may not be a sub-region of the total field of view). In the case of a system where the EUV light is converted into electrons or visible light, the effect of conversion and subsequent imaging systems on the measured aerial image must also be considered with particular regard to degradation of spatial resolution and contrast across the field.

For the purposes of this study we restrict our analysis to points 1-3 above, which enables us to identify preliminary designs which are in principle capable of satisfying the requirements of AIM mode imaging. Full design qualification, which is beyond the scope of this preliminary design review, would require point 4 to be addressed and chould form the first phase of a design and construction project. However points 1-3 should be directly addressed in proposals to build an AIM tool in order to demonstrate that the proposed design is capable of satisfying the requirements of AIM mode imaging.

\subsection{Structure of this report}

To evaluate the performance of each of the candidate systems for an AIM microscope application, and possibly perform further down-selection of candidate system configurations, it is necessary to compare the imaging performance of the proposed systems to that of a production stepper system. Our first step is therefore to define the imaging properties of the stepper system of which we intend to emulate the imaging performance using the AIM microscope.

For imaging AIM mode some form of EUV projection optic is required in order to emulate the imaging characteristics of a stepper system ${ }^{9}$. We therefore evaluate the salient performance characteristics of several objective designs and quantitatively compare this to the anticipated performance of a production stepper system. This enables us to assess the suitability of various objective configurations for satisfying the requirements of AIM mode imaging. Based on this analysis of candidate objectives we proceed to integrate these objectives into system concepts which use both visible light and electron secondary magnification, as well as an all-EUV imaging system. The issues relating to these systems as a whole are discussed, and illumination schemes and throughput budgets discussed.

This report is accordingly divided into the following sections:

1. Background discussion of concepts and metrics applicable to the design study

2. Evaluation of potential EUV objective configurations for satisfying AIM mode requirements

3. System integration, including designs incorporating different secondary magnification options

4. Throughput budget analysis and critical design evaluation

\footnotetext{
${ }^{8}$ If the effect of flare is to be modelled in software the total flare of the AIM optics would have to be less than that of the stepper system to be emulated.

${ }^{9}$ This is true even for a scanning system. A scanning system requires some form of optics in order to provide illumination that emulates the pupil of the stepper system; however, for a scanning system the objective need only be corrected at one field point, greatly simplifying the design and enabling the use of configurations that are unsuitable for full-field imaging application.
} 


\section{Potential EUV objective configurations}

For AIM mode it is essential that the results obtained by measuring the aerial image with an AIM tool provide the same information that would be obtained in a stepper system. Mimicking the performance of a stepper is essential if the results obtained using an AIM tool are to provide a reliable assessment of defect printability in a production tool. Production stepper optics strive to achieve diffraction-limited performance across the field, thus for an AIM tool we also desire a system capable of mimicking this diffraction limited imaging - albeit in magnification rather than reduction system. An important application of an AIM tool is in probing through-focus behaviour of defects for process window analysis, thus it is also essential that the through-focus behaviour of the AIM tool mimic that of a typical stepper system. An EUV imaging objective is required in all potential AIM tool configurations considered in this study. As a first step we consider various EUV objective designs and compare their aerial image performance to that expected of a typical 6-mirror stepper system.

The analysis approach we adopt for evaluation of different designs is as described in section 2.2 above:

1. Satisfying the requirement of $0.0625 \mathrm{NA}$ clear aperture at $6^{\circ}$ angle of incidence using $13.5 \mathrm{~nm}$ EUV light with a bandwidth of $2 \%$;

2. Preliminary system characterisation comparing the incoherent modulation transfer function (MTF), throughfocus behaviour and distortion of the optical system across the field with that of a stepper system across the field; and

3. Aerial image calculation across the field for a characteristic feature size $-20 \mathrm{~nm}$ at the wafer or $80 \mathrm{~nm}$ at the mask, corresponding to spatial frequencies up to $6250 \mathrm{lp} / \mathrm{mm}$ - to demonstrate the equivalence of aerial images measured using the AIM objective with the performance of a 6-mirror stepper system.

Full design qualification, including 2D aerial image calculation incorporating the effects of flare and manufacturing aberrations, is not considered in this section.

\subsection{Metrics of optical system performance: MTF, distortion and through focus behaviour}

Equivalence of the aerial images obtained in an AIM tool with those of a production stepper is the key metric by which to assess the relative performance of different AIM mode configurations. However, as identified in section 2.2 above, there are several other metrics of optical system performance which are useful aids in characterising the performance of different system designs. Excellent performance with regard to these metrics is a necessary but not necessarily sufficient condition in order for a particular design to properly emulate the lithographic-quality imaging properties of a stepper system and provides a useful guide to the performance of a given design.

RMS wavefront error provides an estimate of the amount of design and/or manufacturing aberrations present in an optical system and provides an estimate of the amount of contrast loss expected for a given system. If the overall magnitude of the system wavefront aberration is small it is possible to express the normalised intensity of the image of a point object in the focal region of the lens as being independent of the nature of the aberration, with the normalised intensity being smaller then the ideal value by an amount proportional to the mean square value of the aberrations over the pupil and provides an estimate of anticipated degradation in image contras $\mathrm{t}^{10}$. Excellent contrast transfer is desired in a lithographic system and, thus, also in an AIM tool, hence the magnitude of the composite RMS wavefront error is a useful first estimate of the contrast and imaging quality anticipated of a design ${ }^{11}$.

Distortion is the difference between a linearly scaled version of the object and the actual mapping performed by an objective. Distortion is undesirable in a lithographic camera as it causes errors in the geometry of the printed pattern, which in turn causes features to print in incorrect locations. Distortion over the field has the effect of altering the distance between objects in image space so that measurements of relative object placement and CD no longer directly correspond to that obtained in a lithographic camera. The amount of distortion therefore provides a measure of how well the image is transferred from the mask to the CCD plane. In this study we quote distortion as the maximum departure of image placement from ideal object position, thus a distortion of $9 \mathrm{~nm}$ means that at the worst point in the field, the image is $9 \mathrm{~nm}$ from where it would be located in a distortion-free imaging system.

The Modulation Transfer Function (MTF) is the modulus of the optical transfer function (OTF) which is in turn the Fourier transform of the point spread function. The OTF is independent of the precise nature of the object and is a

\footnotetext{
${ }^{10}$ See, for example, Born and Wolf Principles of Optics, $7^{\text {th }}$ (expanded) edition, p522, §9.1.3.

${ }^{11}$ In analysing the wavefront, and thus determining the RMS wavefront error, it is common practice to consider only the contribution of the first 37 Zernike terms.
} 
measure of the accuracy with which different spatial frequency components in the object are reproduced in the image. Being object-independent the OTF and its modulus the MTF provide a convenient object-independent measure of the performance of an optical system. The incoherent MTF is an excellent guide to anticipated imaging performance and provides a ready method for comparing the performance of different system designs at different spatial frequencies.

One of the major applications of an AIM tool is probing through-focus response of a defect, thus it is important to consider the through-focus response of an AIM tool. The MTF can be computed at different values of defocus to produce a two-dimensional surface representing the contrast transfer of different spatial frequencies as focus is varied, however this is difficult to analyse especially when evaluating performance across the field of view. We therefore compare the contrast transfer at a selected spatial frequency near the resolution limit of a 6-mirror camera, 62501p/mm for different values of defocus. This is done across the field of view to ensure that through-focus response is consistent across the field of view, enabling us to draw conclusions about the extent to which through-focus printability assessment will depend on the location of the object in the field of view.

\subsection{Typical performance of a 6-mirror 0.25NA system}

To enable quantitative comparison of AIM system designs with the image quality expected in a production tool it is necessary to baseline against the expected imaging quality of an EUVL scanner. Surveying the currently available commercial and patent literature it is anticipated that the first production systems will be 6 mirror designs with a numerical aperture of about $0.25 \mathrm{NA}$. There are designs for such systems in the literature and for this study we pick an existing 6-mirror, 0.25NA Hudyma design as representative of the type of EUV system likely to be built. Two such system configurations are shown below in Figure 1. We do not repeat the design details and optical prescriptions here as these details can be found in the patents referenced in Figure 1.

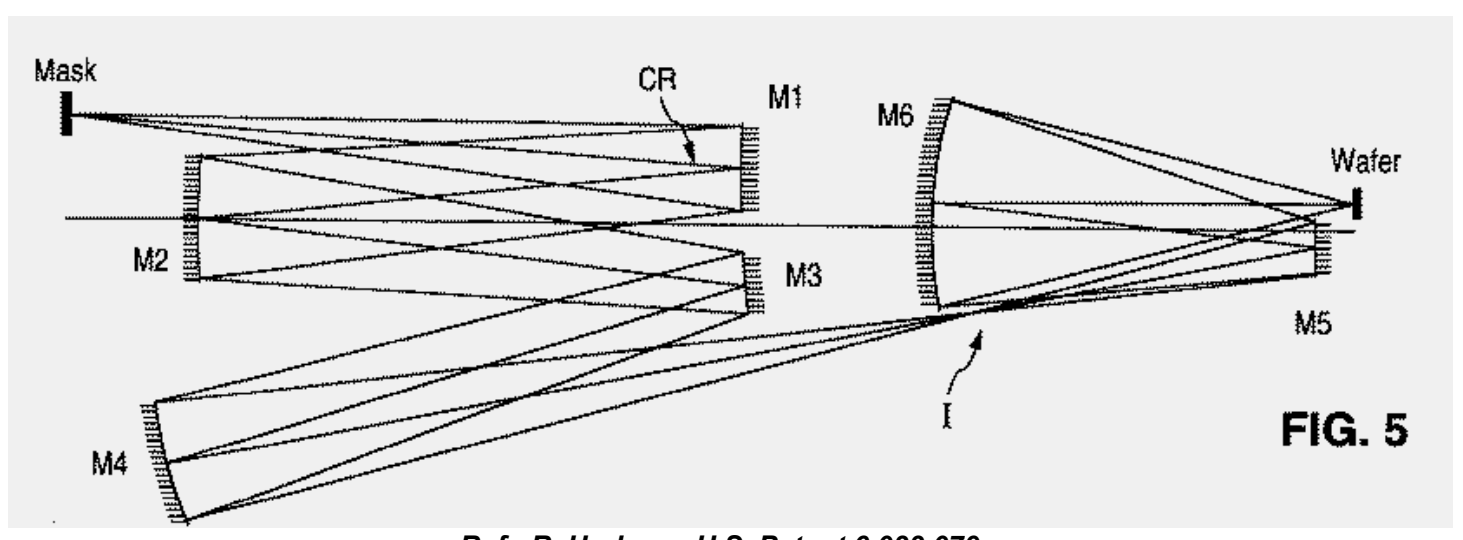

Ref: R. Hudyma, U.S. Patent 6,033,079

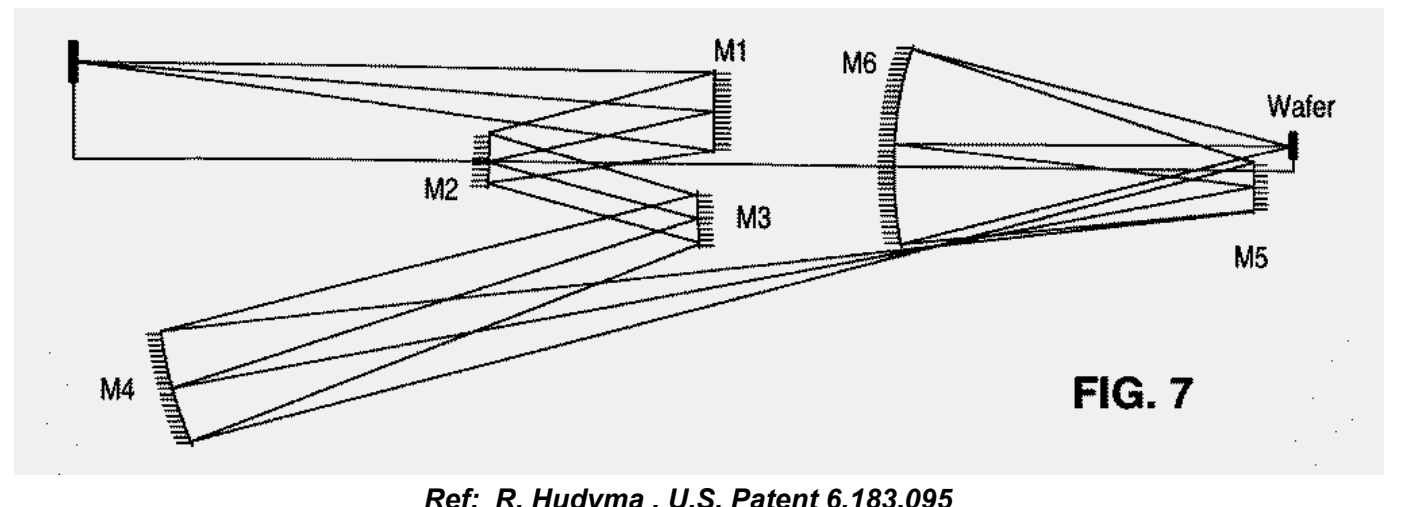

Ref: R. Hudyma , U.S. Patent 6,183,095

Figure 1

Typical 6-mirror EUV projection optic systems with a numerical aperture of $0.25-0.3 \mathrm{NA}$.

The optical performance of these systems approaches the "zero aberration" condition by design across the ring field. Depending upon the actual design form, the field composite root mean square (RMS) wavefront error of these designs varies in a ranges from $0.010 \lambda$ to $0.020 \lambda(\lambda=13.4 \mathrm{~nm})$. The static distortion across the ring field is corrected 
to values less than $0.5 \mathrm{~nm}$ at any point in the ring field, and there is no appreciable concentration of design error in any one particular Zernike term. This high level of aberration correction enables excellent CD control, the best possible depth of focus, and optimum exposure latitude. Performance of actual EUVL scanners will not be limited by the optical design, but by the fabrication errors present on the mirrors.

An EUV AIMS tool must be capable of resolving the as-designed projected imagery from the aforementioned system because it is anticipated that eventually mirror fabrication will improve to the point where the design aberrations become the factor limiting system performance. This high level of performance is illustrated simply by the incoherent MTF and its through-focus behaviour characteristics shown in Figure 2. For good lithography, a diffraction-limited incoherent performance is a necessary, but not sufficient condition for production quality imagery. Rigorous partially coherent aerial image calculations for $30 \mathrm{~nm}$ dense features and $20 \mathrm{~nm}$ isolated features, Figure 3 , confirm that the imaging performance across the field is highly uniform across the whole field exhibiting little or no CD variation by design. Translating this statement into one about printability, when the aerial image is transformed into a circuit pattern by exposing a threshold resist the same feature will show little or no CD variation regardless of where it is located within the imaged field. The same feature will print identically and have the same dimensions regardless of location in the field.

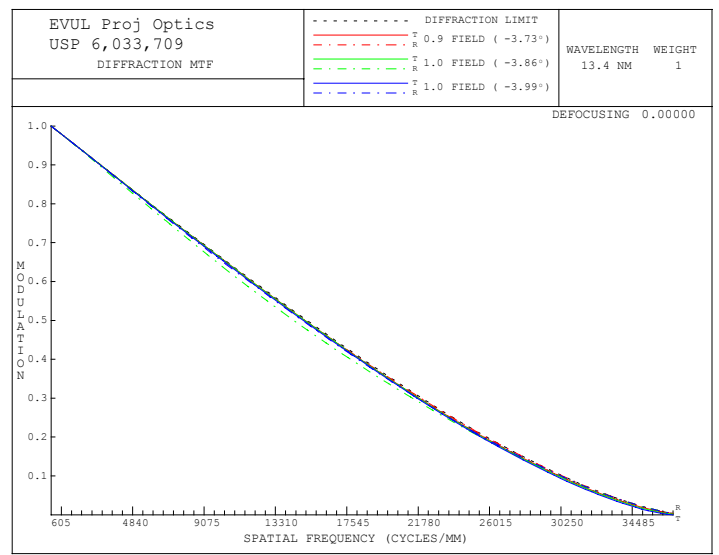

Incoherent modulation transfer function (MTF)

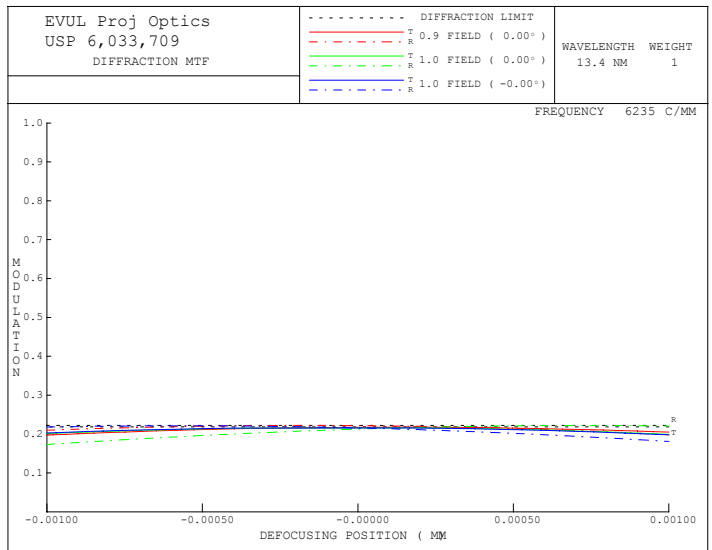

Through-focus behaviour at $6250 \mathrm{lp} / \mathrm{mm}$

Figure 2

Incoherent MTF (left) and through-focus behaviour (right) for a typical 6-mirror projection optic system. For an AIM tool we seek to emulate this diffraction-limited performance across the region quoted as useable for AIM mode imaging.

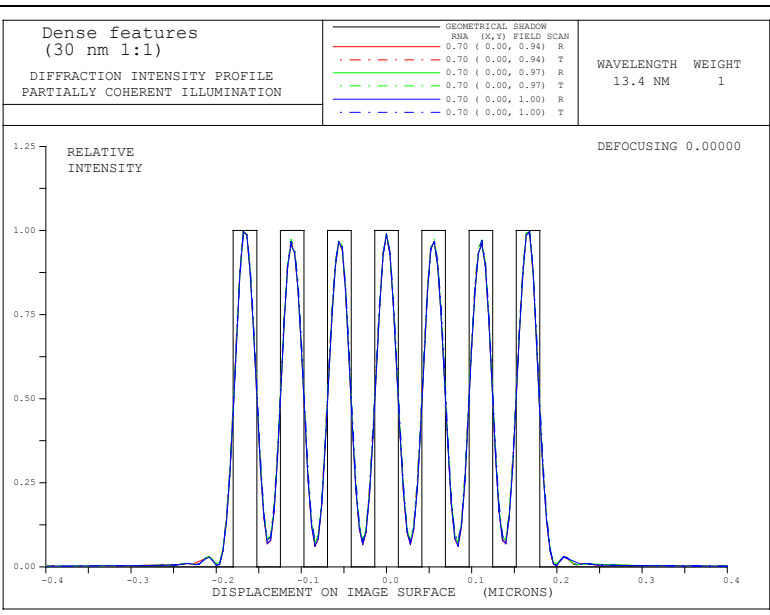

$30 \mathrm{~nm}$ dense features, $1: 1$ pitch $(\sigma=0.7, \lambda=13.4 \mathrm{~nm})$

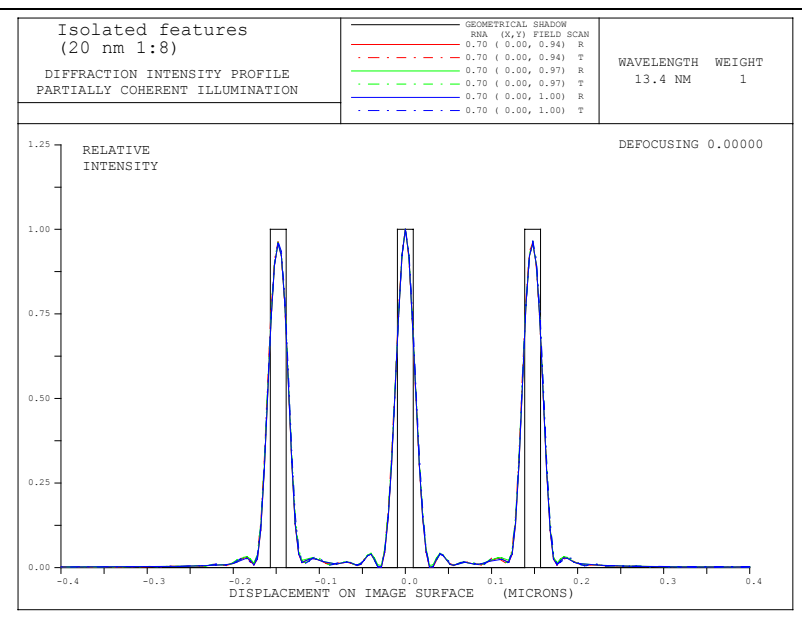

$20 \mathrm{~nm}$ isolated features $1: 8$ pitch $(\sigma=0.7, \lambda=13.4 \mathrm{~nm})$

Figure 3

Design-limited aerial image performance across ring field for typical $0.25 \mathrm{NA}$ 6-mirror system. 


\subsection{Performance and field of view requirements for AIM mode}

For AIM mode it is essential that the results obtained with an AIM tool provide the same information that would be obtained in a stepper system. This requirement, discussed in detail in section 2.2 above, means that the optics of a AIM system have to mimic the optical performance of a stepper.

An AIM tool is, however, intended for defect review rather than lithographic printing thus it is not immediately obvious or apparent that lithographic-quality aerial images performance is necessary across the entire field of view. It may be acceptable to design an AIM microscope in which the imaging properties emulate that of a stepper system only across a central portion of the field used for measurement, with the remainder of the field used for navigation and defect location rather than AIM measurement. There is some attraction to adopting this approach because it is easier to design and manufacture a system corrected over a small field of view, although the limitations of adopting this approach should be understood and appreciated.

\subsubsection{Variable aerial image performance across the field and CD analysis}

To analyse printability a threshold resist model is typically applied to the measured aerial image intensity in order to convert the aerial image intensity into a virtual circuit pattern that can be used for analysing the effect of a defect on the printed feature size (CD). The use of a threshold model makes data analysis, and therefore conclusions about CD variation, highly sensitive to small changes in aerial image intensity. The effect of having varying optical performance across the field is to vary the aerial image across the field, thus different conclusions about CD would be drawn from the same object depending on where it is located within the field of view. This effect is illustrated in Figure 4 below; when imaging is corrected across the field of view (left) applying a threshold resist leads to the same conclusions regarding CD and printability regardless of where the object is located in the field of view, whilst in the case of imaging with variable performance across the field (right) conclusions regarding $\mathrm{CD}$ and printability will differ depending on where the measurement is made within the field of view.

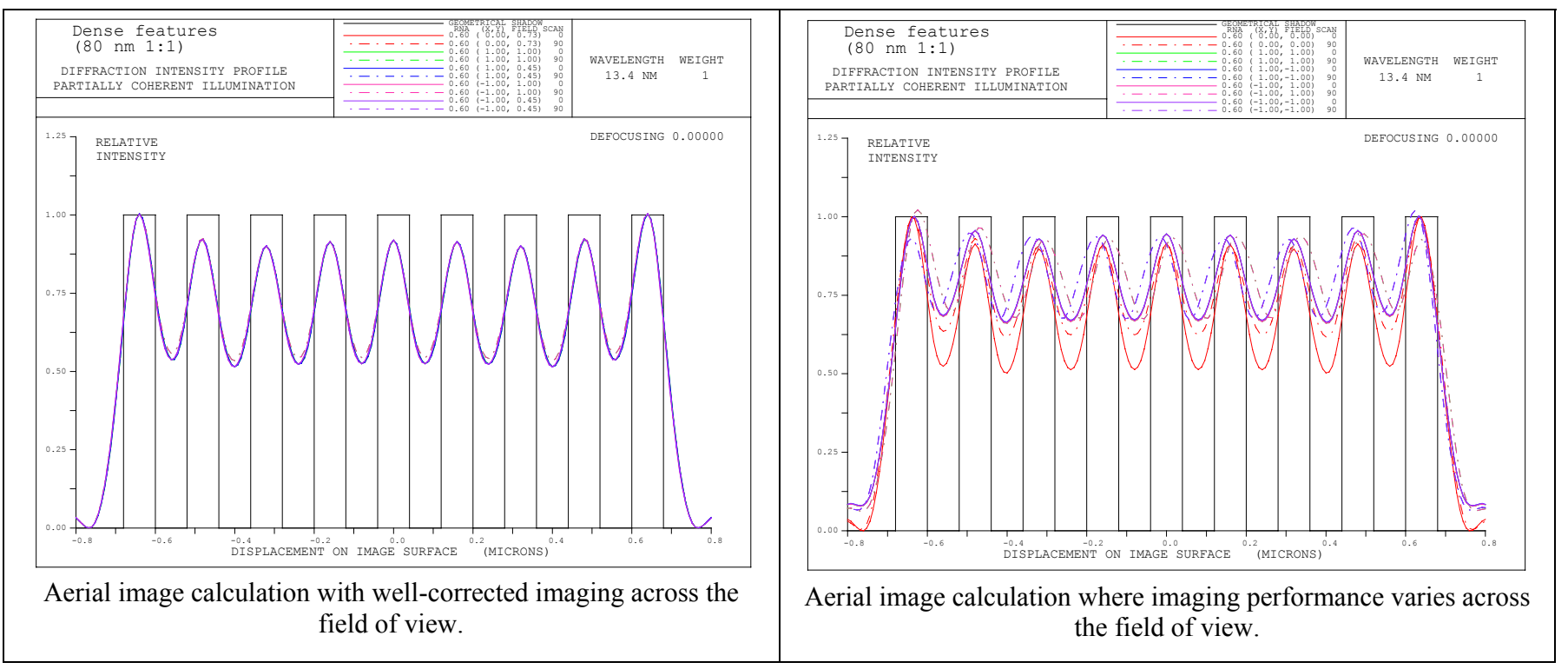

Figure 4

Illustration of well-corrected imaging across the field (left) compared to a system where performance varies across the field (right). 


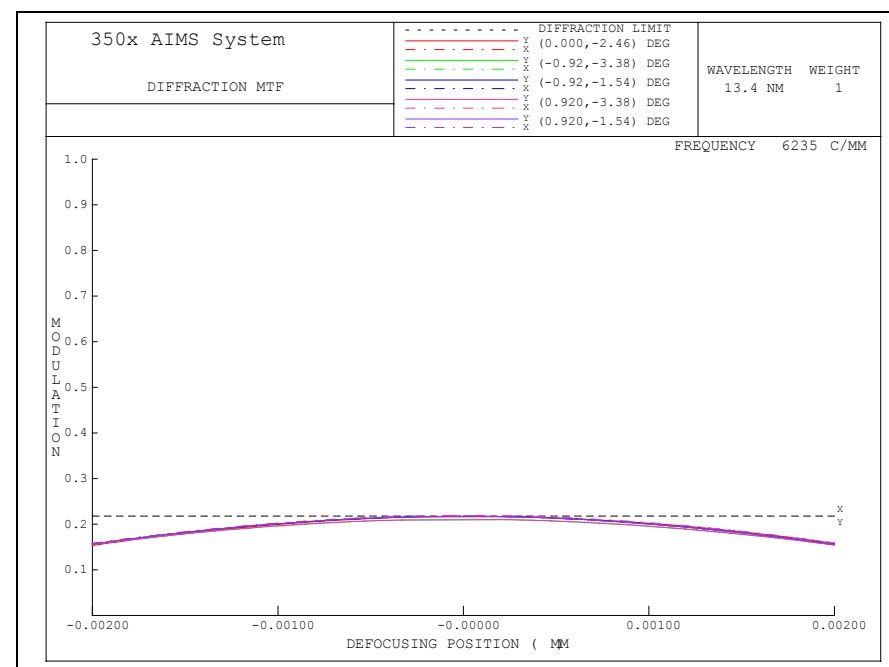

Performance through focus at $62501 \mathrm{p} / \mathrm{mm}$ showing consistent through-focus performance across the imaged field.

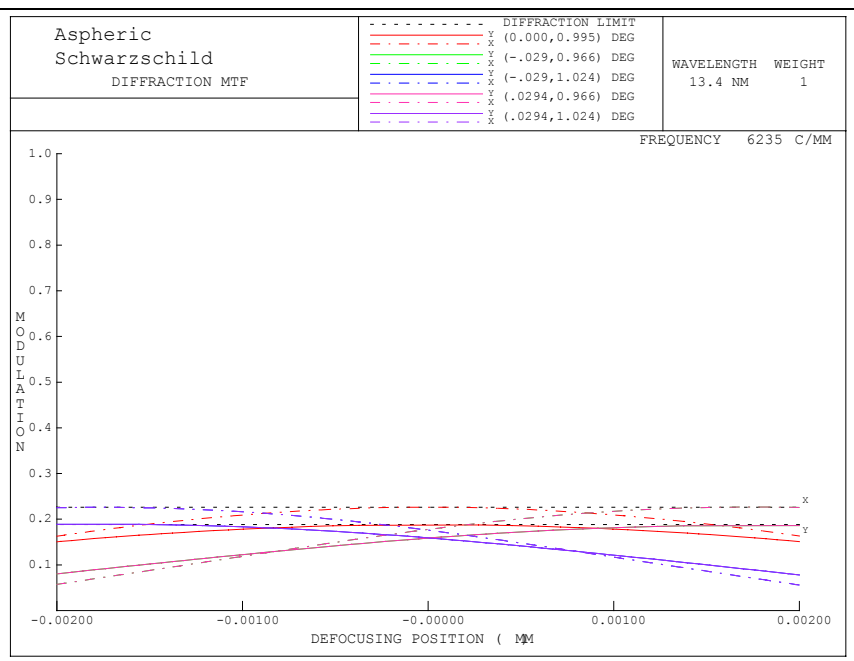

Performance through focus at $6250 \mathrm{lp} / \mathrm{mm}$ for an optical design where through-focus imaging performance varies across the imaged field.

Figure 5

Illustration of uniform through-focus behaviour across the field (left) compared to a system where through-focus performance varies across the field (right).

\subsubsection{Distortion across the field and its effect on circuit analysis}

The amount of distortion allowed across the field of view is another optical design parameter where there is some choice for an AIM system as to degree of constraint placed on the optical design. Distortion is typically displayed as the change in shape of a regular grid projected through the optical system and is undesirable in a lithographic printing system, particularly a scanning system where the object passes through field of view as it is printed. The significance of distortion is that magnification varies across the field; the shape of the object is not preserved and will appear to be different depending on where the object is located in the field. An example of distortion in a lens is shown in Figure 6. In the context of a AIM applications the presence of significant distortion will cause the result of a CD measurement to depend on where the object is placed in the field of view. Distortion also affects distance measurements made on the image: in the presence of distortion the measured image distance between two features on the mask will depend on where those two features are located in the field. The presence of excessive distortion will inhibit the comparison of microscope images with predicted feature geometry stored in a database, and the amount of acceptable distortion should be negotiated between the instrument maker and user.

One issue is whether distortion has to be corrected across the entire field of view or only over a portion of the imaged field. As with contrast variations across the field the idea is that distortion needs be corrected only across a central portion of the field used for measurement whilst the remainder of the field is used for locating the defect rather than performing measurements. And once again the effects of this on CD analysis and potential restrictions on the useability of an AIM tool for a given application must be carefully considered and understood before a decision is made to accept significant distortion across the field. 


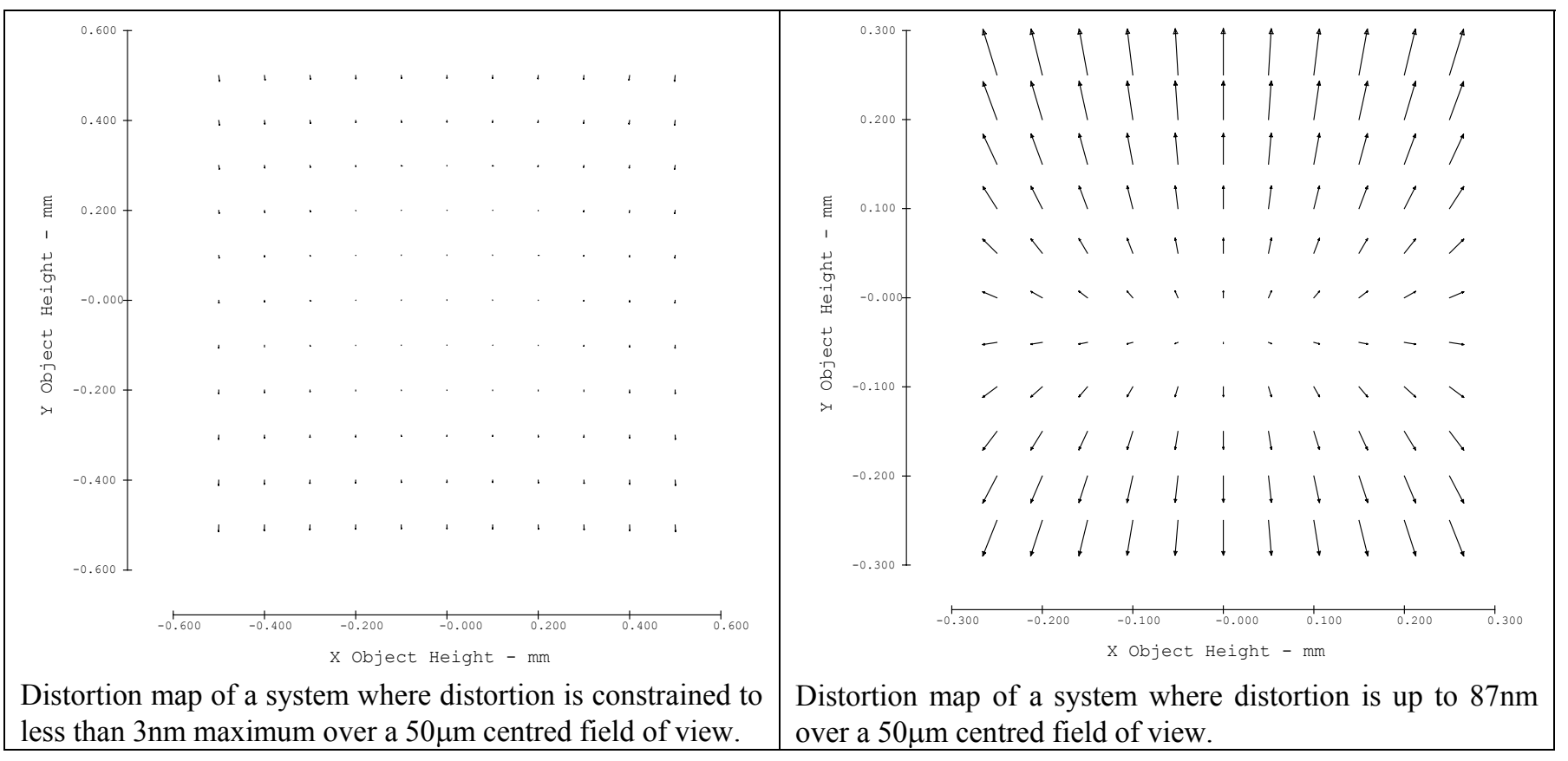

Figure 6

Examples of distortion maps for a system with distortion corrected across the field (left) and where there is significant distortion (right). Distortion produces a warped version of the object; the shape of the object is not preserved and will appear to be different depending on where the object is located in the field. If distortion is present the result of CD and distance measurements will depend on where the object is placed within the field of view.

\subsubsection{Field of view trade-offs and requirements for design qualification}

It is possible to design, and a vendor may propose, an AIM mode solution in which distortion and/or contrast vary over the field of view. This may be an acceptable design compromise for an AIM tool in the light of a cost-benefit analysis. However, for such a design it is important to distinguish between the visible field of view - the size of the image in a single frame - and the sub-portion of the field of view over which accurate CD measurements can be made. In a system employing a design with variable performance across the field of view it is essential that the sub-field useable for AIM-quality measurements be clearly identified and that AIM-quality imaging performance be demonstrated over that sub-field. In practice this means that aerial image performance must be characterised at both the best and worst field points within the quoted AIM field.

For the purposes of this study, however, we seek to pursue a solution in which the whole field is properly corrected so that AIM measurements can be made with confidence regardless of where the object is located in the field of view. Our goal in pursuing such an approach is twofold: to assess the feasibility of designing such a system, and because a tool with well-corrected imaging across the field provides the least opportunity for drawing the wrong conclusions about defect printability by virtue of image placement within the field of view. 


\subsection{Existing objective designs for AIM mode}

It would be advantageous to be able to use an existing lens design for AIM mode imaging, thus we first examine existing EUV camera designs to see if they can be adapted for use in AIM mode. For the purposes of this study we examine three generic systems: an all-spherical Schwarzschild system, an aspheric Schwarzschild system, and an equal radii design. These are respectively analogous to the 10x optics at Sandia, the BEL optics and the MET optics. The analysis we provide here is, however, generic and equally applicable to designs of a similar type. This analysis should therefore be viewed as a technology survey of many different ideas for an AIM microscope implementation.

\subsubsection{Family of two-mirror systems}

The class of two-mirror systems, illustrated in Figure 7, have been well studied in the literature. Whilst there are many possible two-mirror configurations, for the purposes of an AIM tool the requirements of having magnification, a physical working distance, and both a real object and image restricts the class of possible solutions applicable to an AIM microscope. In this report we examine various Schwarzschild and two-mirror systems of the MET type. We investigate the classic Schwarzschild, which has mirrors of radii $(1+\sqrt{5}) f$ and $(1-\sqrt{5}) f$, and also explore Schwarzschild derivatives of the same general geometry but with different mirror combinations. Designs similar to the MET based on the equal-radii concept are also explored; strictly speaking the MET is a particular design which has been fabricated for use as a microstepper projection optic. Whilst we explore using the existing MET design, MET-type objectives of different configurations are also explored.

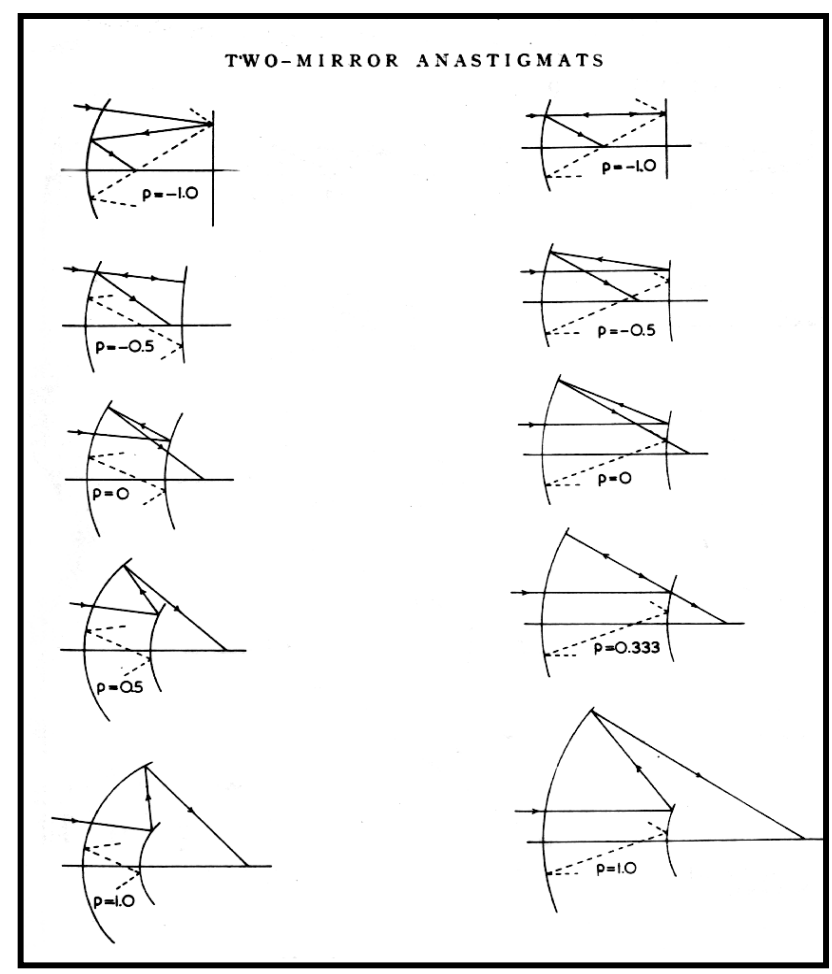

Figure 7

Family of two-mirror anastigramtic designs, taken from Wynne, JOSA 1959. For an AIM system only a few of these configurations are feasible. 


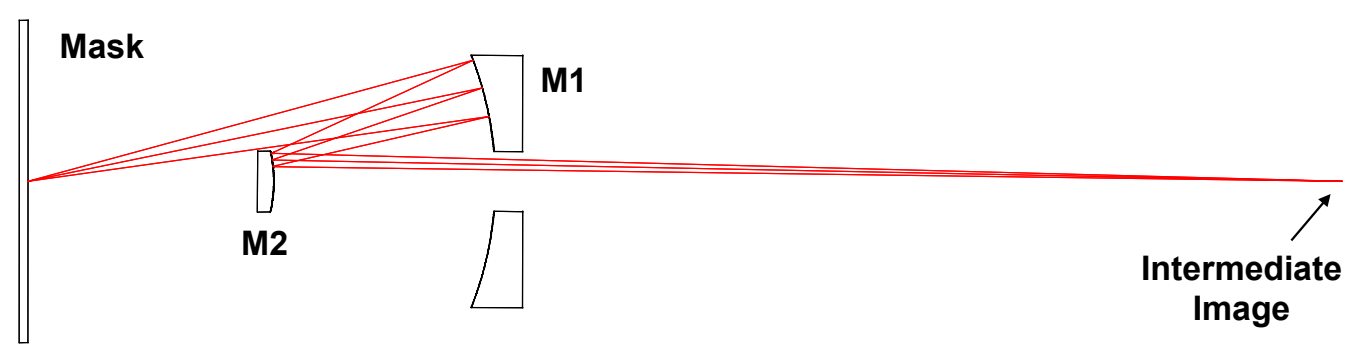

\begin{tabular}{|c|c|c|c|}
\hline Parameter & Value & Parameter & Value \\
\hline Wavelength $(\lambda)$ & $13.4 \mathrm{~nm}$ & Composite RMS & $55 \mathrm{~m} \lambda(0.008 \mathrm{~nm})$ \\
\hline Magnification & $10 x$ & Distortion (max) & $87 \mathrm{~nm}$ \\
\hline Numerical aperture & 0.0625 & Aspheric departure & None \\
\hline Field & $50 \mu \mathrm{m} \times 50 \mu \mathrm{m}$ & Mask angle & $11.84^{\circ}$ \\
\hline Total track & $600 \mathrm{~mm}$ & Working distance & $>100 \mathrm{~mm}$ \\
\hline
\end{tabular}

Figure 8

Schwarzschild objective design consisting of all spherical mirrors.

We start our investigation with a classic Schwarzschild design consisting of all-spherical mirrors as illustrated in Figure 8 above. The classic Schwarzschild design has mirrors of radii $(1+\sqrt{5}) f$ and $(1-\sqrt{5}) f$ and has wellcorrected imaging on-axis for an object at infinity. For AIM mode imaging we use this design with a $0.0625 \mathrm{NA}$ clear aperture to one side of the secondary mirror (M2), and employ a design with $100 \mathrm{~mm}$ working distance and a total track length of a $600 \mathrm{~mm}$ giving a magnification of 10x. As designed the optical axis is perpendicular to the mask, thus to ensure adequate clearance past the M2 mirror the chief ray has an angle of incidence on the mask of 11.8 degrees. Due to constraints of the Schwarzschild design this is the minimum angle of incidence that can be achieved with an on-axis Schwarzschild system with mirrors of radii close to $(1+\sqrt{5}) f$ and $(1-\sqrt{5}) f$.

To evaluate the performance of this system we compute the incoherent MTF of the system across the field, Figure 9. A high-NA (0.25NA) stepper will have diffraction-limited performance out to $6250 \mathrm{lp} / \mathrm{mm}$ at the mask, corresponding to $80 \mathrm{~nm}$ features on the mask and $20 \mathrm{~nm}$ features on the wafer, thus we also plot the MTF of this system out to $6250 \mathrm{lp} / \mathrm{mm}$. Although imaging in a stepper will be partially coherent, we evaluate the incoherent MTF as excellent performance with incoherent imaging is a necessary condition for having excellent partially coherent imaging performance. The composite RMS wavefront error is $55 \mathrm{~m} \lambda$ suggesting that this system should have reasonable contrast tranfer, however note from the MTF plot that the contrast transfer is limited by diffraction at high spatial frequencies and aberrations for mid spatial frequencies. Note the loss of contrast in mid-spatial frequencies - as described above this is unacceptable for AIMS mode as variation in contrast will map directly into printability assessment measurements made with this objective would yield different results to that obtained in a stepper system, violating the fundamental requirement of AIM mode imaging. Note also the significant variation in contrast transfer across the field which would lead to different conclusions on printability depending on where the object was located in the field of view. This design also has significant variation in through-focus behaviour across the field illustrated in Figure 9 which shows the through-focus contrast at $6250 \mathrm{lp} / \mathrm{mm}(20 \mathrm{~nm}$ at the wafer). This shows that through-focus behaviour varies significantly across the field, thus conclusions on printability will differ depending on where the object is located in the field of view. Finally we compute the distortion across the field for this imaging system, with a map of distortion across the field shown in Figure 10. The distortion ranges up to $87 \mathrm{~nm}$ within the field of view, which would have a significant impact on CD measurement and feature metrology depending on where the feature is located in the field of view. Because of the poor imaging performance and incorrect angle of incidence at the mask we are therefore driven away from adopting a spherical Schwarzschild design for the purposes of this study. 

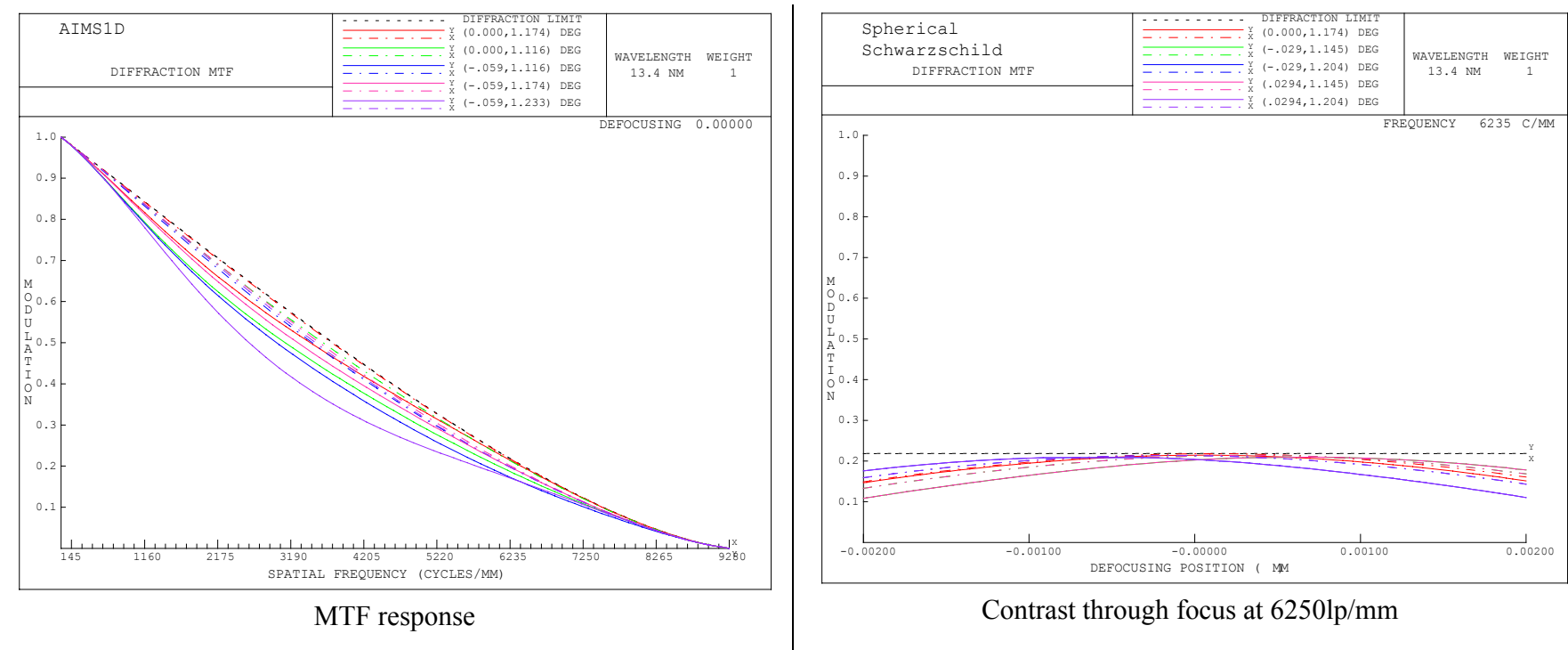

Contrast through focus at $6250 \mathrm{lp} / \mathrm{mm}$

Figure 9

Incoherent MTF and through-focus response at $62501 \mathrm{p} / \mathrm{mm}$ for an all-spherical Schwarzschild system. Note the aberration and diffraction limited contrast transfer in mid and high spatial frequencies, and the variation in contrast transfer across the field. Note also that the through-focus behaviour also varies significantly across the field.

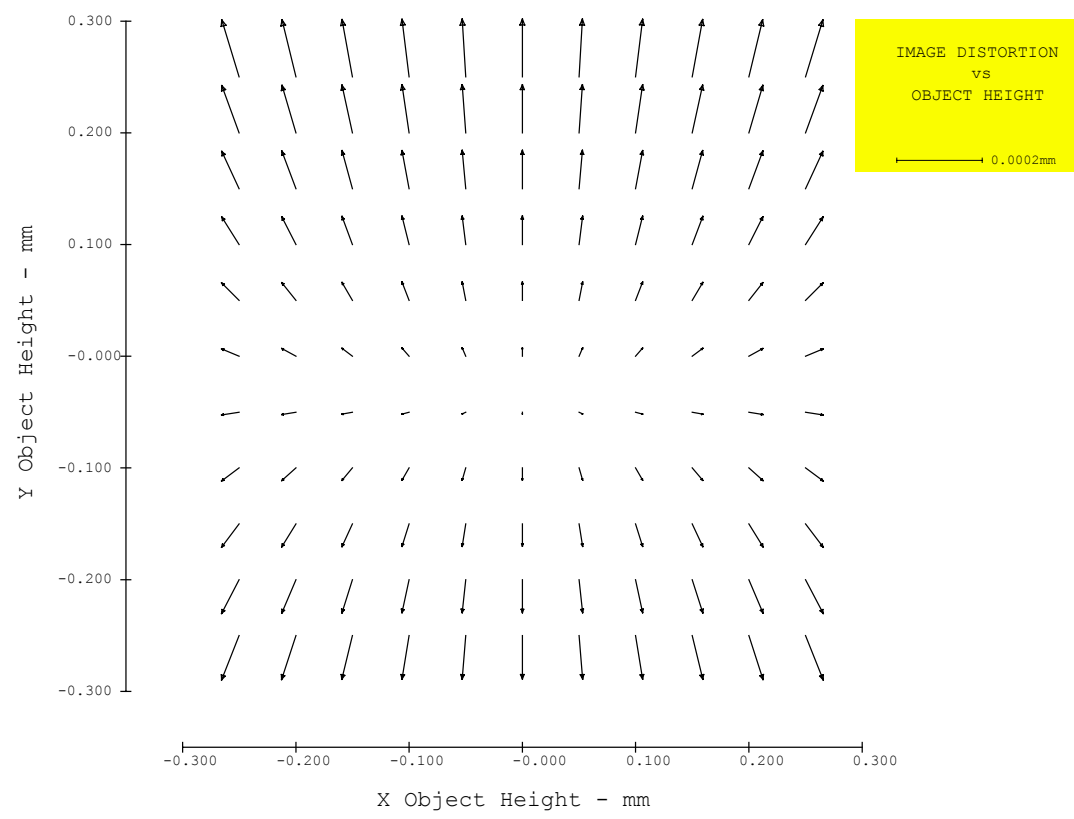

Figure 10

Field distortion for an all-spherical Schwarzschild system; maximum distortion in the field of view is $87 \mathrm{~nm}$.

Several spherical two-mirror Schwarzschild systems that depart from the $(1+\sqrt{5}) f$ and $(1-\sqrt{5}) f$ prescription were also considered for an AIM mode implementation. The challenge is to find a geometry where the chief ray has an input angle of $6^{\circ}$ and at the same time has an off-center cone with an NA $=0.0625$. We developed two design solutions: the first one, shown in Figure 11 below, departs only slightly from the canonical prescription and has the required input angle of $6^{\circ}$ but only an unobstructed NA of 0.040 . By departing more from the canonical prescription, we developed a second design, shown in Figure 12 below, with better clearance about the small secondary mirror. This design has the required $\mathrm{NA}=0.625$; however in order to avoid obstruction by the secondary mirror the range of input 
angles is between $4.8^{\circ}$ and $11.3^{\circ}$ and centered at $7.5^{\circ}$. Using an angle of $7.5^{\circ}$ instead of $6^{\circ}$ shifts the reflectivity maximum about $0.04 \mathrm{~nm}$ and impart additional shadowing effects on the aerial imagery. Also note that the beam footprint on the smaller secondary mirror is reduced in diameter relative to the beam footprint on the larger primary mirror, indicating increased fabrication challenges with this approach.

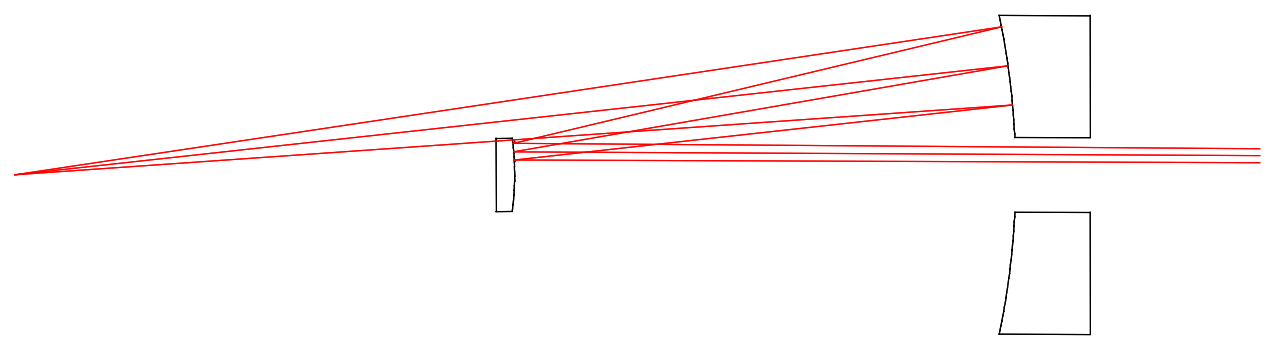

Figure 11

AIM mode implementation with clear aperture of $\mathrm{NA}=0.040, \mathrm{AOI}=6.2^{\circ}$. The full NA of the parent Schwarzschild is $0.24 \mathrm{NA}$.

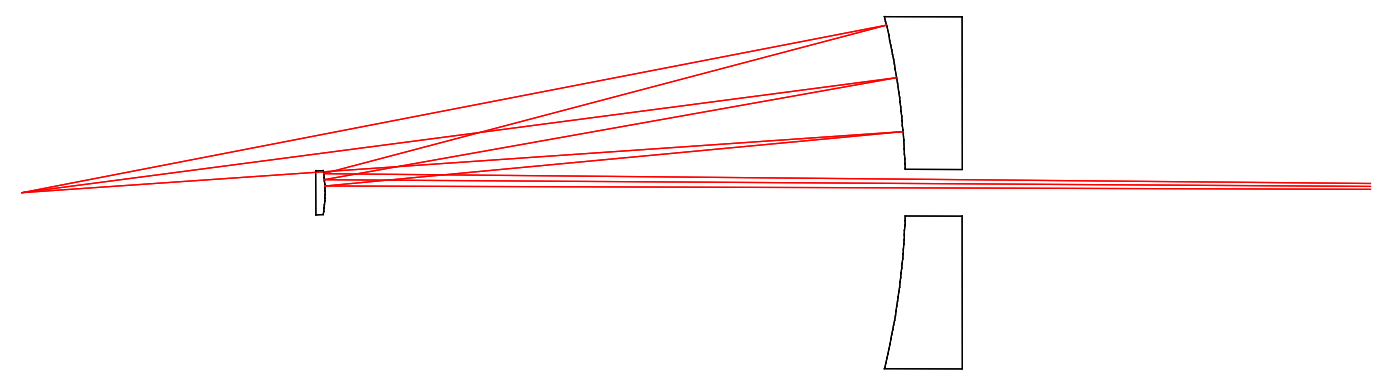

Figure 12

AIM mode implementation with clear aperture of $\mathrm{NA}=0.063, \mathrm{AOI}=7.9^{\circ}$. Full NA of the parent optic is $0.20 \mathrm{NA}$.

By scaling down this design it is possible to obtain a magnification of $440 \mathrm{x}$ in a track length of $2 \mathrm{~m}$, as illustrated in Figure 13 below. However scaling down the design also requires further reducing the mirror size, increasing the fabrication challenges associated with this approach. The question then becomes one of whether it is possible to fabricate such small mirrors to EUV specifications. Note also that the angle of incidence is not $6^{\circ}$ at the mask by design.

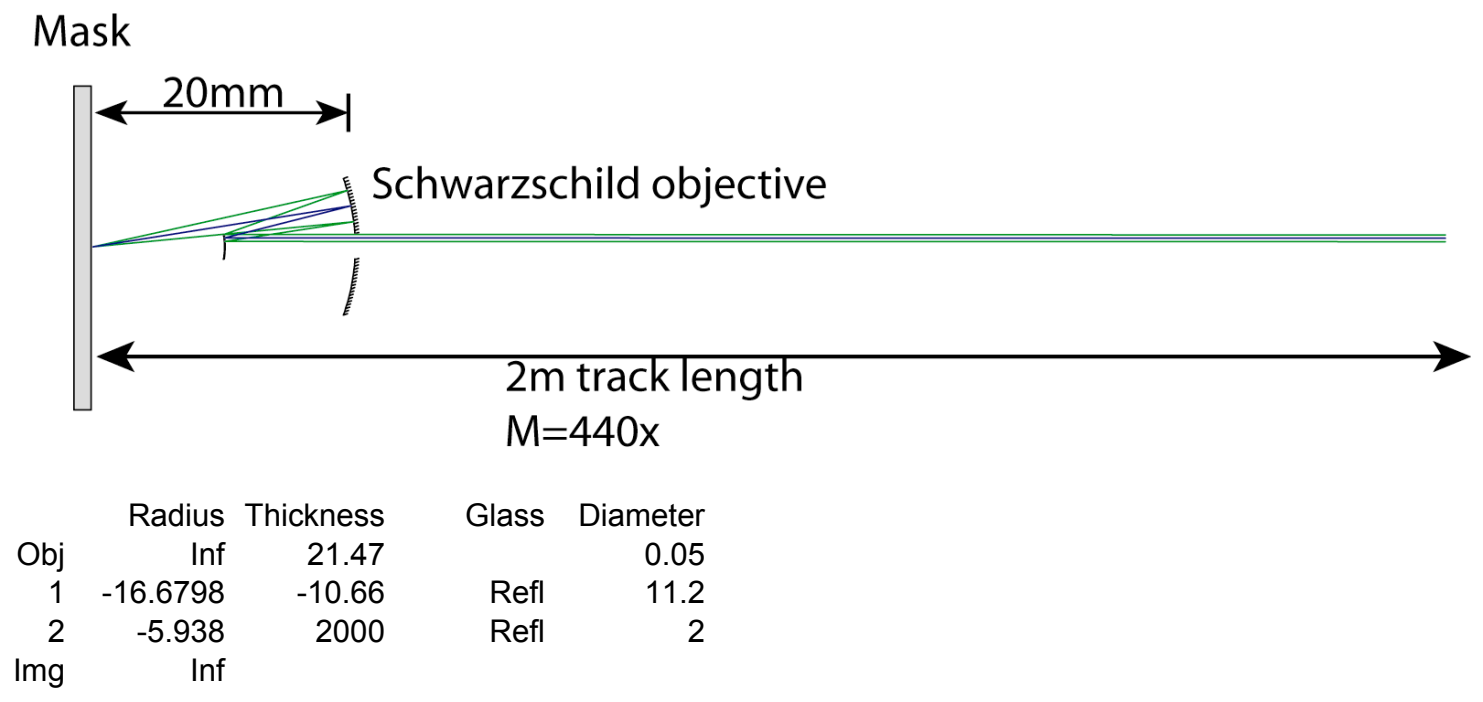

Figure 13

Scaled-down version of the Schwarzschild objective to provide 440x magnification in a $2 \mathrm{~m}$ track length. 


\subsubsection{Schwarzschild system with mild aspheric components}

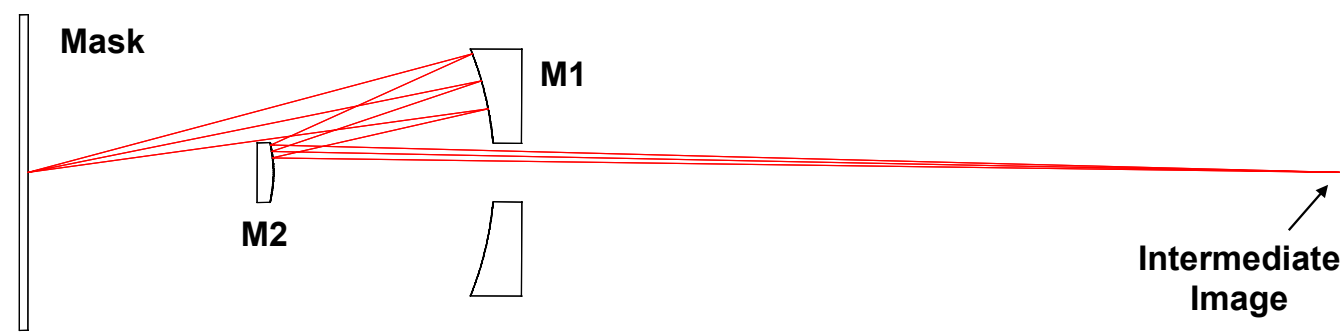

\begin{tabular}{|c|c|c|c|}
\hline Parameter & Value & Parameter & Value \\
\hline Wavelength $(\lambda)$ & $13.4 \mathrm{~nm}$ & Composite RMS & $0.6 \mathrm{~m} \lambda(0.008 \mathrm{~nm})$ \\
\hline Magnification & $10 \times$ & Distortion (max) & $3 \mathrm{~nm}$ \\
\hline Numerical aperture & 0.0625 & Aspheric departure & $<1 \mu \mathrm{m}$ \\
\hline Field & $50 \mu \mathrm{m} \times 50 \mu \mathrm{m}$ & Mask angle & $11.84^{\circ}$ \\
\hline Total track & $600 \mathrm{~mm}$ & Working distance & $>100 \mathrm{~mm}$ \\
\hline
\end{tabular}

Figure 14

Schwarzschild objective design with mild $(<1 \mu \mathrm{m})$ aspheric components

For the reasons described above we are driven to consider the effect of introducing mild aspheric components to the Schwarzschild design to compensate for the loss of contrast and through-focus performance observed in the all spherical system. This design is similar to the spherical system described above in that it has a $0.0625 \mathrm{NA}$ clear aperture to one side of the secondary mirror (M2), $100 \mathrm{~mm}$ working distance and a total track length of a $600 \mathrm{~mm}$ giving a magnification of 10x. However mild aspheric departure of $<1 \mu \mathrm{m}$ is introduced into both mirrors to correct imaging performance. Once again the optical axis is kept perpendicular to the mask, thus to ensure adequate clearance past the M2 mirror the chief ray has an angle of incidence on the mask of 11.8 degrees. Due to constraints of the Schwarzschild design this is the minimum angle of incidence that can be achieved with an on-axis Schwarzschild system ${ }^{12}$.

Once again we plot the incoherent MTF and through-focus behaviour at $6250 \mathrm{lp} / \mathrm{mm}$ in order to evaluate the performance of this design. The composite RMS wavefront error is $0.6 \mathrm{~m} \lambda$ which indicates good contrast transfer at the central field point. With the introduction of mild aspheres the incoherent MTF, Figure 15, is now diffraction-limited across the 50x50 $\mu \mathrm{m}$ field of view. Through focus response, also shown in Figure 15, is also uniform across the field. Note also that the introduction of mild aspheres into the design has corrected the geometric distortion in this system to $3 \mathrm{~nm}$ maximum across the field of view. This is significantly less than the anticipated pixel size, thus this system as designed has adequate distortion control for AIM applications. However, the problem of the 11.8 degree chief ray angle of incidence at the mask remains - this angle of incidence violates the 6 degree angle of incidence AIM condition and is a fundamental limitation of the on-axis Schwarzschild design. As previously described changing the angle of incidence necessitates a change in wavelength (to remain in resonance with the multilayer) and means that effects such as geometrical shadowing are not properly captured. We are therefore led to consider workarounds including tilting the objective in order to obtain the correct angle of incidence at the mask.

\footnotetext{
${ }^{12}$ Note that the possibility of tilting the objective, and the effect on the image properties, will be considered later on in this report.
} 


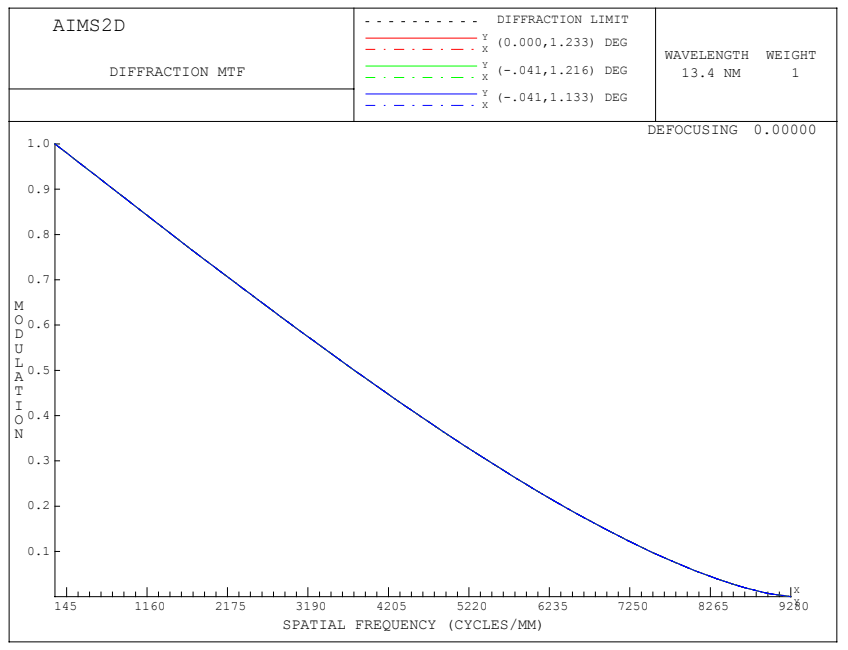

MTF response

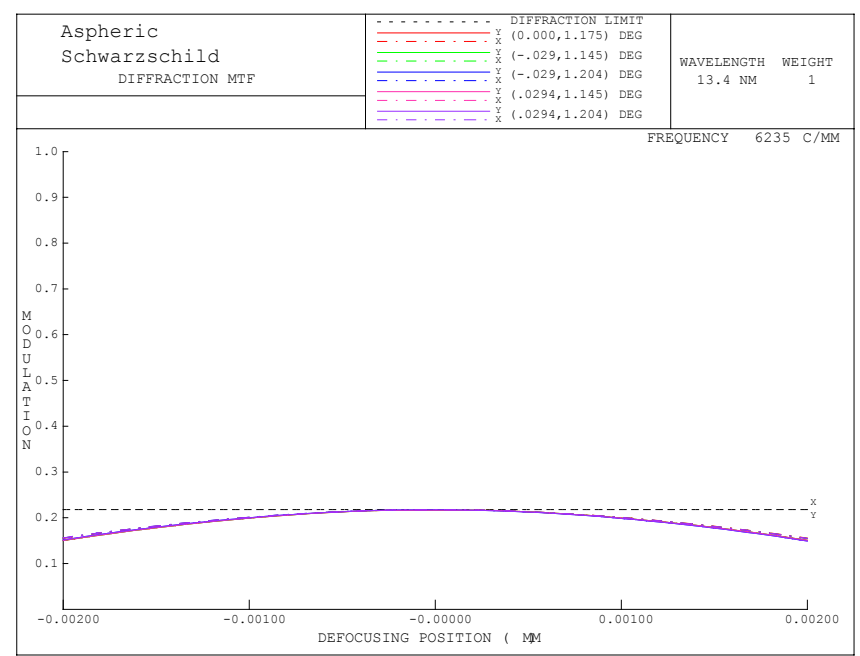

Contrast through focus at $6250 \mathrm{lp} / \mathrm{mm}$

Figure 15

Incoherent MTF and through-focus response at $62501 \mathrm{p} / \mathrm{mm}$ for a Schwarzschild system with mild $(<1 \mu \mathrm{m})$ aspheric mirrors. Note that imaging is diffraction-limited across the field and that through-focus behaviour is also uniform across the field. This is desirable for AIM mode, however the angle of incidence of the chief ray at the mask is limited by the Schwarzschild design to 11.8 degrees, violating the AIM imaging condition.
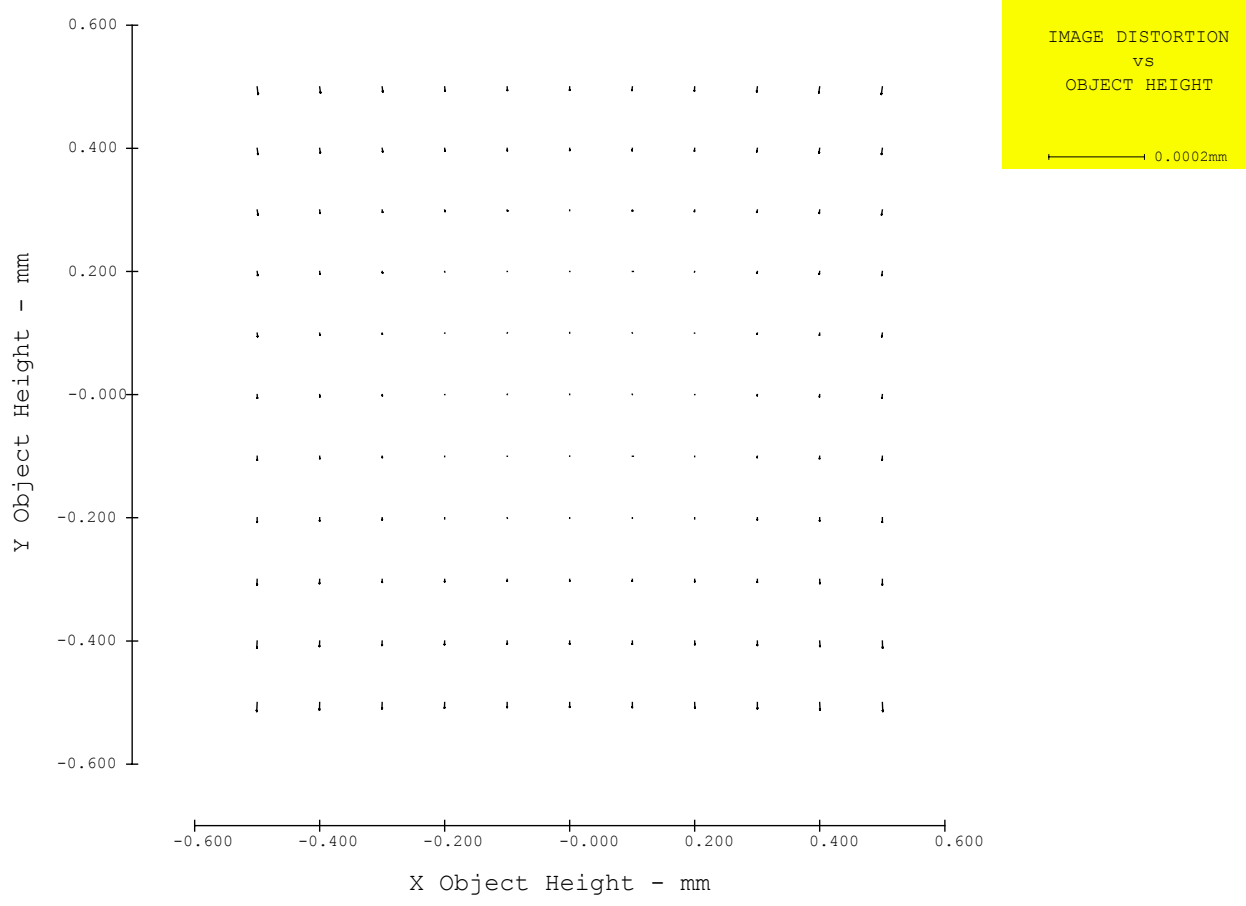

Figure 16

Field distortion for an all-spherical Schwarzschild system; maximum distortion in the field of view is $3 \mathrm{~nm}$, an acceptably small amount for AIM microscope applications. 


\subsubsection{Tilted Schwarzschild designs to capture 6 degree angle of incidence}

In order to correctly capture shadowing effects and to avoid having to use a different wavelength to that of a stepper system we investigate the possibility of solving the angle of incidence problems inherent in the Schwarzschild design through the use of a tilted objective. To do this the illumination angle at mask is set to $6^{\circ}$ and the optical system is tilted $4-6^{\circ}$ with respect to the mask in order to accommodate the $6^{\circ}$ angle of incidence. Tilting the objective with respect to the mask causes optimal imaging to occur on a tilted image plane (the Scheimpflung condition), however it is possible to also consider the case where the imaging plane is parallel to the objective. We consider both cases below.

\subsubsection{Image performance on a plane parallel to objective}

The case of imaging to a plane parallel to the objective is shown schematically in Figure 17 below:

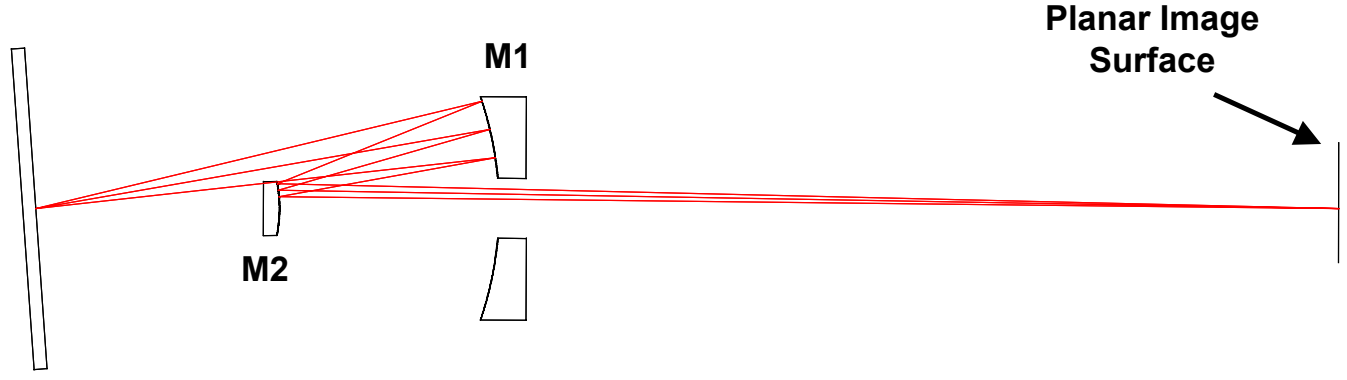

\begin{tabular}{|c|c|c|c|}
\hline Parameter & Value & Parameter & Value \\
\hline Wavelength $(\lambda)$ & $13.4 \mathrm{~nm}$ & Composite RMS & $66 \mathrm{~m} \lambda(0.88 \mathrm{~nm})$ \\
\hline Magnification & $10 \mathrm{x}$ & Distortion (max) & $246 \mathrm{~nm}$ \\
\hline Numerical aperture & 0.0625 & Aspheric departure & $<1 \mu \mathrm{m}$ \\
\hline Field & $50 \mu \mathrm{m} \times 50 \mu \mathrm{m}$ & Mask illumination & $6.0^{\circ}$ \\
\hline Total track & $600 \mathrm{~mm}$ & Tilt angle & $4.0^{\circ}$ \\
\hline
\end{tabular}

Figure 17

For our analysis we use the same aspheric Schwarzschild design as above so as to enable direct comparison with the on-axis imaging performance. First of all we note that tilting the objective with respect to the mask leads to an increase in composite RMS wavefront error to $66 \mathrm{~m} \lambda$, a factor of 100 worse than the $0.6 \mathrm{~m} \lambda$ present in the centred optical system. This immediately tells us that contrast transfer is going to be degraded by using a tilted objective. Note also that with this approach distortion across the $50 \times 50 \mu \mathrm{m}$ field is increases to $245 \mathrm{~nm}$ in the form of keystone distortion, compared to $3 \mathrm{~nm}$ for the centred design discussed above. This is equivalent to many pixels in object space, thus the effect of distortion in the image to $\mathrm{CD}$ and distance measurements is expected to be significant across the field of view for this configuration.

Inspection of the system MTF, Figure 19, shows serious degradation in contrast transfer in the plane of best focus and also shows that there is significant variation in optical response across the field of view; the central field point is close to diffraction limited whilst points at the edge of the field are seriously degraded. This significant variation in behaviour across the field is also apparent in the through-focus response at $6250 \mathrm{lp} / \mathrm{mm}$, Figure 18 , which shows significant variation in behaviour across the field of view. This variation in performance across the field is demonstrated in the aerial image calculation at $6250 \mathrm{lp} / \mathrm{mm}$, Figure 19: applying a threshold resist model to this data would give significantly different conclusions about printability depending on where the object was located in the $50 \times 50 \mu \mathrm{m}$ field of view.

As described above, for the purposes of this design study we seek a solution for AIM mode in which imaging emulates the performance of a stepper across the field of view in order to demonstrate the feasibility of such a system, thus we look for a way in which to restore imaging performance using a tilted objective design. 


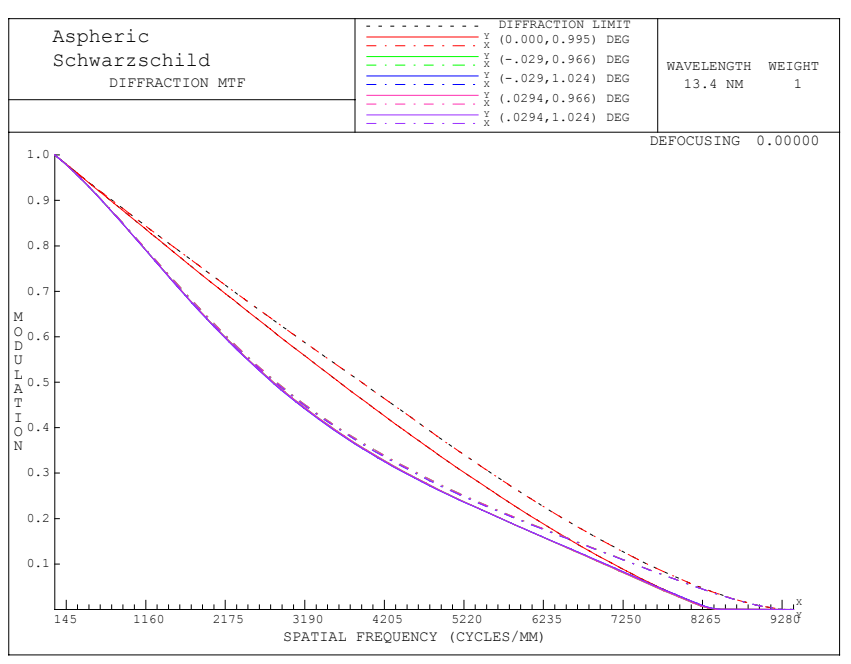

MTF response in plane of best focus

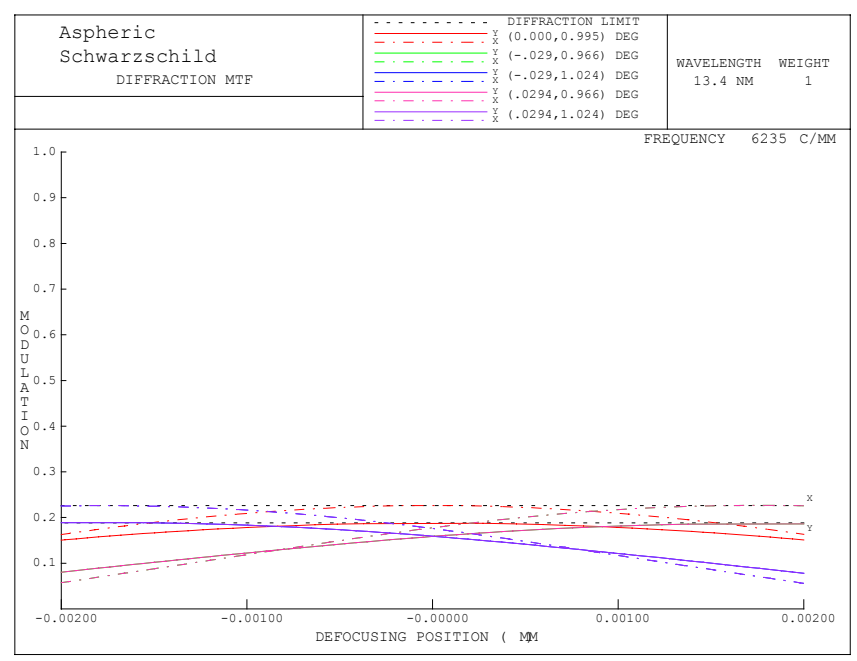

Contrast through focus at $62501 \mathrm{p} / \mathrm{mm}$ is severely degraded

Figure 18

Incoherent MTF and through-focus response at $6250 \mathrm{lp} / \mathrm{mm}$ for a Schwarzschild system with mild $(<1 \mu \mathrm{m})$ aspheric mirrors tilted with respect to the mask by 4 degrees in order to fully capture the AIM pupil. Note the significant variation in contrast transfer across the field, and the asymmetric and field-dependent variation in through-focus response at $6250 \mathrm{lp} / \mathrm{mm}$ on the mask.

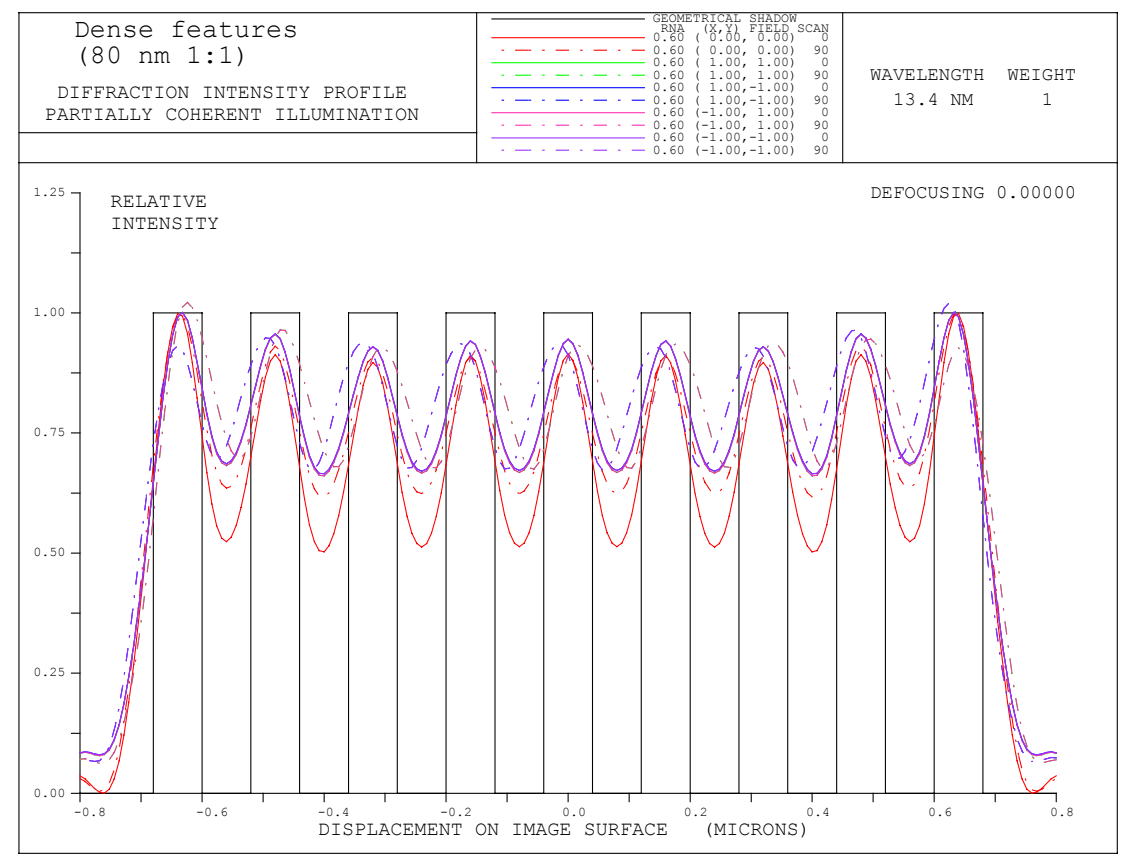

Figure 19

Aerial image simulation at $6250 \mathrm{lp} / \mathrm{mm}$ on the mask illustrating the effect of the field-dependent variation in contrast transfer caused by tilting the objective by 4 degrees with respect to the mask in order to fully capture the AIM pupil. 


\subsubsection{Imaging performance on a tilted image plane}

The Scheimpflung condition teaches that a tilted object is properly imaged to a tilted image plane, thus we seek to restore the degradation in imaging performance caused by tilting the objective by measuring the image over a tilted image plane. This is illustrated schematically in Figure 20 below.

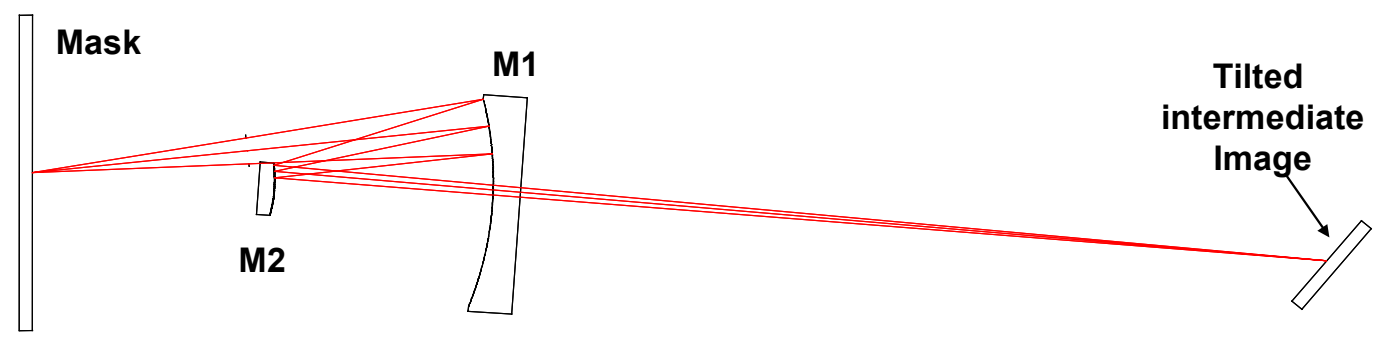

\begin{tabular}{|c|c|c|c|}
\hline Parameter & Value & Parameter & Value \\
\hline Wavelength $(\lambda)$ & $13.4 \mathrm{~nm}$ & Composite RMS & $1.9 \mathrm{~m} \lambda(0.025 \mathrm{~nm})$ \\
\hline Magnification & $10 \times$ & Distortion (max) & $5833 \mathrm{~nm}$ \\
\hline Numerical aperture & 0.0625 & Aspheric departure & $<1 \mu \mathrm{m}$ \\
\hline Field & $50 \mu \mathrm{m} \times 50 \mu \mathrm{m}$ & Mask illumination & $6.0^{\circ}$ \\
\hline Total track & $600 \mathrm{~mm}$ & Tilt angle & $4.0^{\circ}$ \\
\hline
\end{tabular}

Figure 20

Solving the $6^{\circ}$ degrees angle of incidence problem with tilts: illumination angle at mask is set to $6^{\circ}$ and the optical system is tilted 4-

$6^{\circ}$ with respect to the mask in order to accommodate this angle of incidence. Observing the Scheimpflung condition leads to an image plane tilted at 40 degrees for 10x magnification.

According to the Scheimpflung condition imaging performance is restored on an object plane that is tilted by the magnification times the tilt on the object plane, thus for 4 degrees of mask tilt at 10x magnification we get an image plane that is tilted at 40 degrees. This restoration in performance can be seen from the MTF, Figure 21, which is now close to diffraction limited across the field in the plane of best focus. This is confirmed by the aerial image simulations at $6250 \mathrm{lp} / \mathrm{mm}$ shown in Figure 21 which also show little variation in performance across the field at best focus. The composite RMS wavefront error of this design, which is now $1.8 \mathrm{~m} \lambda$ across the field, also indicates how imaging performance has been improved through using a tilted image plane.

Note, however, that whilst the in-focus behaviour is promising there are a number of drawbacks to adopting this approach. Whist imaging performance is restored in the plane of best focus the through-focus response, Figure 22, shows significant variation in performance across the field. Whilst more manageable on a tilted image plane than on a plane aprallel to the objective, the response is still asymmetric and displays significant variation across the field. As one of the main applications of an AIM system is probing through-focus response this type of through-focus response is undesirable. Note also that imaging on a tilted image plane introduces a large amount of keystone distortion. As designed, imaging onto the tilted image plane introduces a maximum distortion of $5833 \mathrm{~nm}$ across the field of view in the form of keystone distortion. This amount of distortion is clearly unacceptable for an AIM application. Lastly, imaging on tilted plane is accompanied by a reduction in effective depth of focus; the tolerance on focus is tightened, thus the image is much more sensitive to small errors in tilt and position of the mask compared to an on-axis design. This restriction in depth of focus may have practical consequences for implementing this design. 


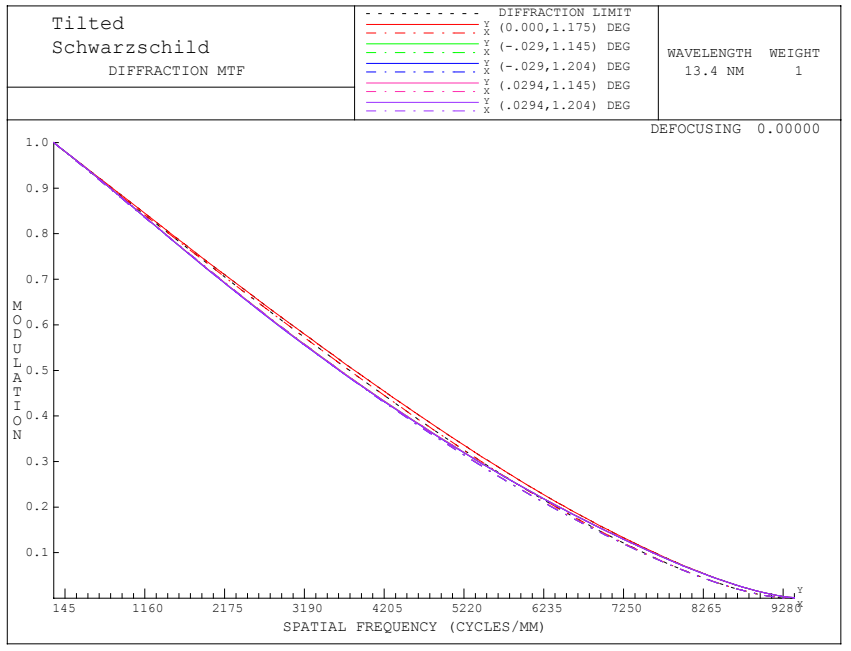

MTF response on tilted image plane

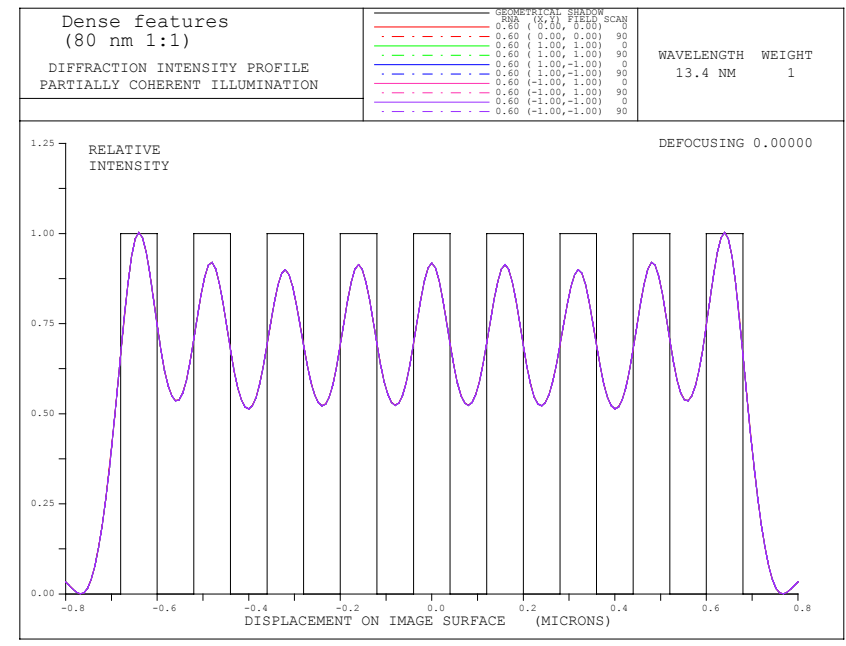

Partially coherent imaging of 80nm (at mask) features, $\sigma=0.6$.

Figure 21

Incoherent MTF and through-focus response at $6250 \mathrm{lp} / \mathrm{mm}$ for a Schwarzschild system with mild $(<1 \mu \mathrm{m})$ aspheric mirrors imaging onto a tilted image plane. Note that imaging is close to diffraction-limited across the field, as illustrated by the aerial image simulations at $6250 \mathrm{lp} / \mathrm{mm}$ across the field.

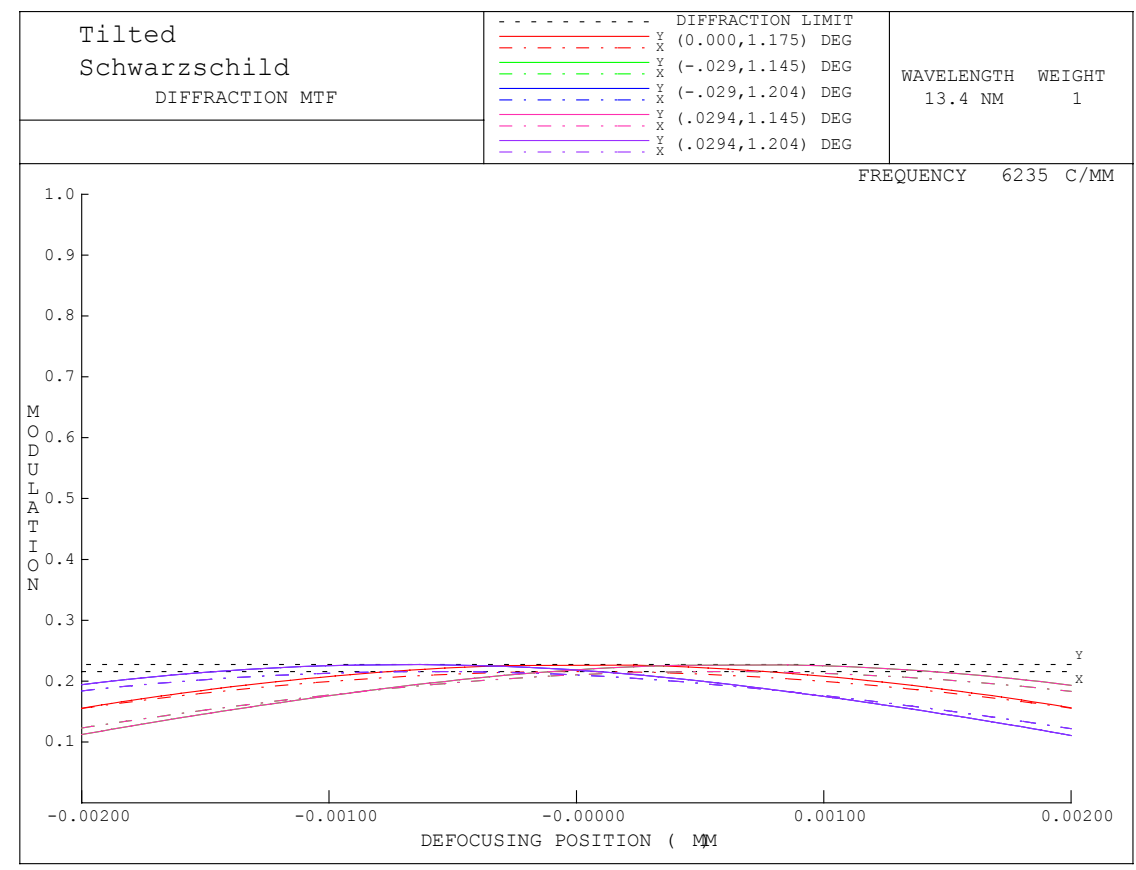

Figure 22

Tilting the image plane restores imaging performance in the plane of best focus, but through focus behaviour on the tilted image plane at $62501 \mathrm{p} / \mathrm{mm}$ is non-uniform across the field and asymmetric with respect to defocus. 


\subsubsection{MET-based system}

As described in the down-selection report the MET is an existing 0.3NA 5x magnification projection lens designed for use in a $200 \times 600 \mu \mathrm{m}$ field of view microstepper with lithographic-quality image correction across the field of view. Although of low magnification and encompassing a higher field of view than desired for an AIM tool the production path for the MET is well-defined thus it is worth investigating whether it is possible to adapt this lens for use in AIM mode.

The basic design of the MET as used in the microstepper is shown below in Figure 23.

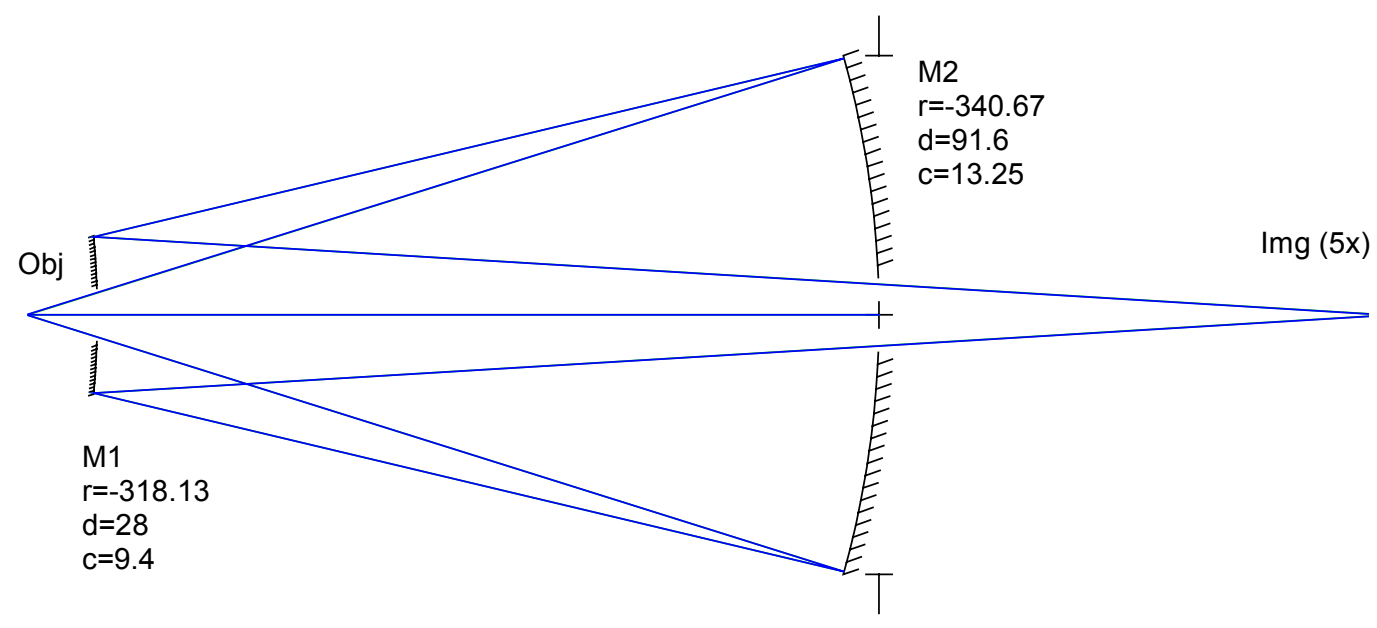

Figure 23

Basic design of the 0.3NA MET microstepper objective.

Applying a 0.0625 NA AIM pupil at 6 degrees at the mask, and placing the mask where the wafer is normally located so that the system provides $5 \mathrm{x}$ magnification rather than $5 \mathrm{x}$ reduction we find that there is significant pupil obscuration resulting from the size of the cutout in M1 - a large proportion of the rays from the AIM pupil back through the M1 aperture. The amount of pupil obscuration can be significantly reduced by resizing the cutouts in both the M1 and M2 mirrors. If the central hole in M1 is reduced from $9.4 \mathrm{~mm}$ to $5 \mathrm{~mm}$ and the hole in M2 is reduced from $13.25 \mathrm{~mm}$ to $8 \mathrm{~mm}$ it is possible to significantly reduce the amount of obscuration as shown in Figure 24, however some pupil obscuration remains.

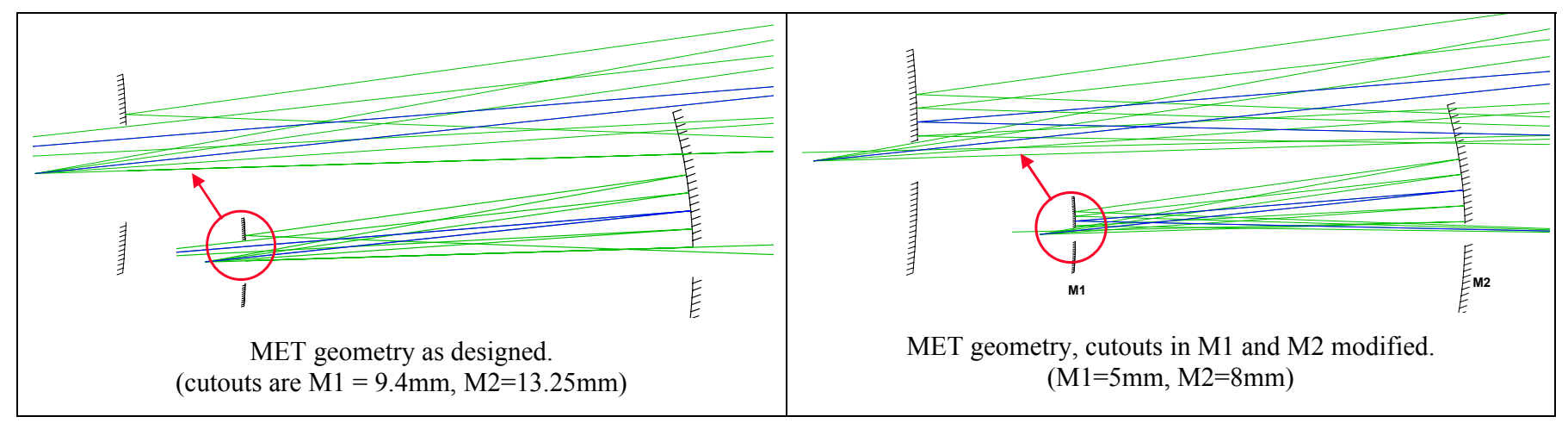

Figure 24

As designed the MET suffers from significant pupil obscuration in AIM mode due to the size of the hole cur in M1 (left). Resizing the apertures in both M1 and M2 to accommodate the AIM pupil significantly decreases but does not eliminate the amount of obscuration caused by the cutout in M1. Note that decreasing the size of the cutout in this way precludes operation at full $0.3 \mathrm{NA}$ as the M1 mirror now blocks the marginal rays. 
The amount of obscuration that remains is very small and motivates the search for a workaround that enables the full $0.0625 \mathrm{NA}$ AIM pupil to be captured. This can be done if the angle of incidence at the mask is changed by $1^{\circ}$ from $6^{\circ}$ to $7^{\circ}$ and the AIM is restricted to emulating a $0.25 \mathrm{NA}, 4 \mathrm{x}$ stepper, giving $0.0625 \mathrm{NA}$ facing the mask. The ray diagram with these modifications applied is shown in Figure 25, from which it can be seen that the whole AIM pupil is now captured by the objective. The change in angle of incidence requires a small shift in the wavelength of the EUV illumination from $13.5 \mathrm{~nm}$ to $13.47 \mathrm{~nm}$ to remain in resonance with the multilayer. There will also be a small change in shadowing effects caused by the change in angle of incidence that must be considered. Both the changes in wavelength and angle of are very small and may be acceptable for an AIM tool. However, the effects of both of these changes that must be modelled as a part of full design qualification to ensure that all salient features on the mask are properly captured. Detailed aerial image calculation, combined with electromagnetic simulations of the mask response, including effects of absorber height, must demonstrate equivalence of the aerial images obtained using this system with that of a production stepper.

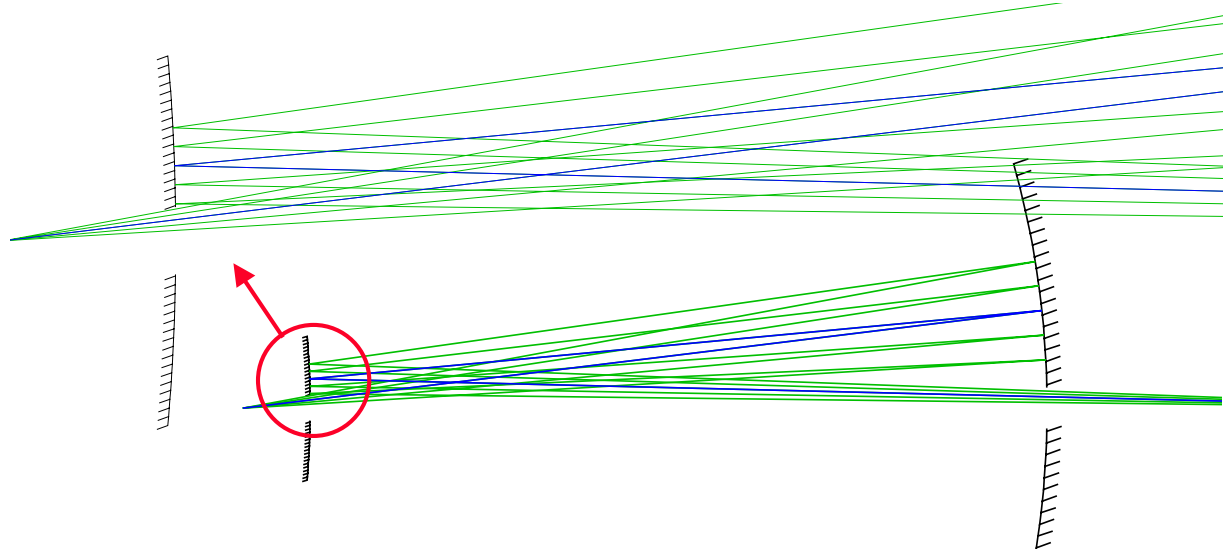

Figure 25

Workaround for the MET objective where mirror cutouts are resized and the working AIM aperture is restricted to emulating a $0.25 \mathrm{NA}, 4 \mathrm{x}$ stepper $(0.0625 \mathrm{NA}$ facing the mask). In addition to resizing the mirror the angle of incidence at the mask is changed from $6^{\circ}$ to $7^{\circ}$ degrees accompanied by a small wavelength shift from $13.5 \mathrm{~nm}$ to $13.47 \mathrm{~nm}$.

An advantage of this design over the Schwarzschild approaches described above is that the objective observes the mask at normal incidence, thus the deleterious effects of working with tilted object and image planes are avoided. The MET design has been qualified for lithographic-quality performance through focus over a $600 \times 200 \mu \mathrm{m}$ field. Because this design is used at normal incidence, when used in AIM mode the performance will also be diffractionlimited across the field. Thus we would anticipate excellent AIM performance using this approach provided the consequences of changing the angle of incidence are properly studied and characterised. 


\subsection{Novel objective design for AIM mode}

Because of the compromised performance anticipated for a tilted Schwarzschild design and the requirement to change angles of incidence if the MET objective is used we seek a solution that enables the AIM condition to be satisfied we proceed to design a novel objective suitable for AIM mode imaging with the motivation to do this is to demonstrate the feasibility of designing such a system. We also note that the MET has a much larger field than required for AIM mode and are therefore motivated to seek a simpler solution with less aspheric departure, more compact optics and a potentially easier manufacturing path.

Starting with an equal radii design of the same family as the MET it is possible to design an objective with excellent imaging performance capable of satisfying the requirements of AIM mode imaging.

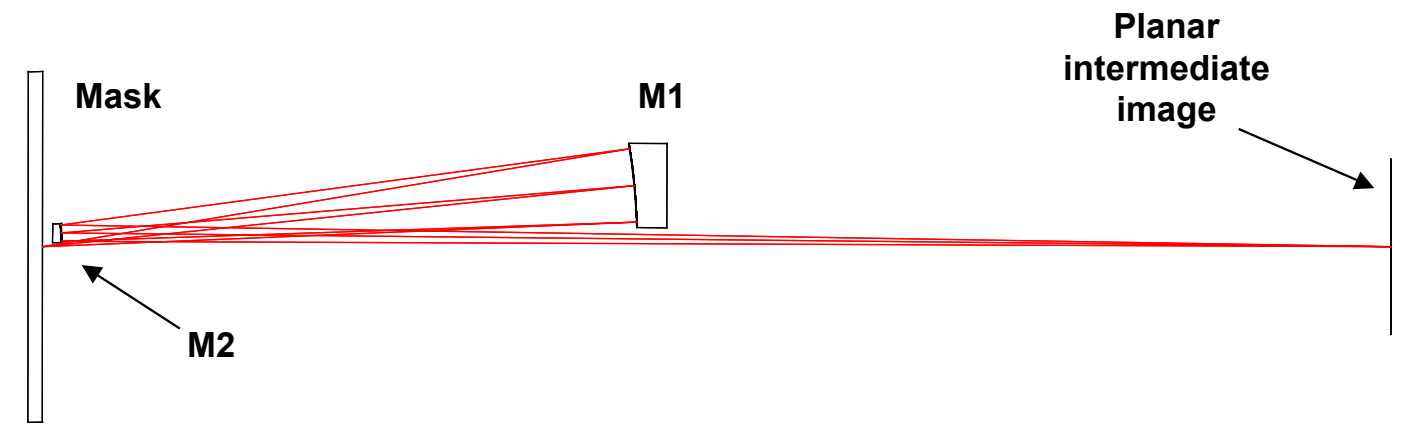

\begin{tabular}{|c|c|c|c|}
\hline Parameter & Value & Parameter & Value \\
\hline Wavelength $(\lambda)$ & $13.4 \mathrm{~nm}$ & Composite RMS & $0.6 \mathrm{~m} \lambda(0.008 \mathrm{~nm})$ \\
\hline Magnification & $10 \times$ & Distortion (max) & $3 \mathrm{~nm}$ \\
\hline Numerical aperture & 0.0625 & Aspheric departure & $<1 \mu \mathrm{m}$ \\
\hline Field & $50 \mu \mathrm{m} \times 50 \mu \mathrm{m}$ & Mask illumination & $6.0^{\circ}$ \\
\hline Total track & $600 \mathrm{~mm}$ & Working distance & $2-4 \mathrm{~mm}$ \\
\hline
\end{tabular}

Figure 26

Equal-radii objective design for AIM mode imaging.

This particular design uses mild aspheric mirrors with $<1 \mu \mathrm{m}$ aspheric departure on all optical surfaces, and has a total magnification of $10 \mathrm{x}$ over a $600 \mathrm{~mm}$ track length, although preliminary investigation suggests that the design should be scaleable over the range of approximately $5 \mathrm{x}$ to $20 \mathrm{x}$ magnification. The composite RMS wavefront error for this system is $0.6 \mathrm{~m} \lambda$ leading to excellent contrast transfer through the system, as illustrated by the MTF plot and through-focus behaviour curves shown in Figure 27 which demonstrate consistent diffraction-limited imaging performance across a 50x50 $\mathrm{m}$ field of view. Note also that the distortion of $3 \mathrm{~nm}$ maximum over the entire field is very low, thus we do not expect any distortion-induced changes in $\mathrm{CD}$ or distance metrology with this design. The clear pupil in this particular design is $0.0625 \mathrm{NA}$ at 6 degrees angle of incidence so shadowing effects on the mask are properly captured and the wavelength matches that of a production stepper system. This, combined with the excellent overall imaging performance, leads us to anticipate that this objective design would faithfully capture the imaging performance of a stepper system across the field of view. Conclusions about printability and CD would be the same regardless of where the object was placed in the field of view, and being diffraction limited across the field we would expect this objective to faithfully capture the salient imaging effects of a stepper across the whole field of view. We therefore select this design for more detailed analysis and system integration in the next section. 


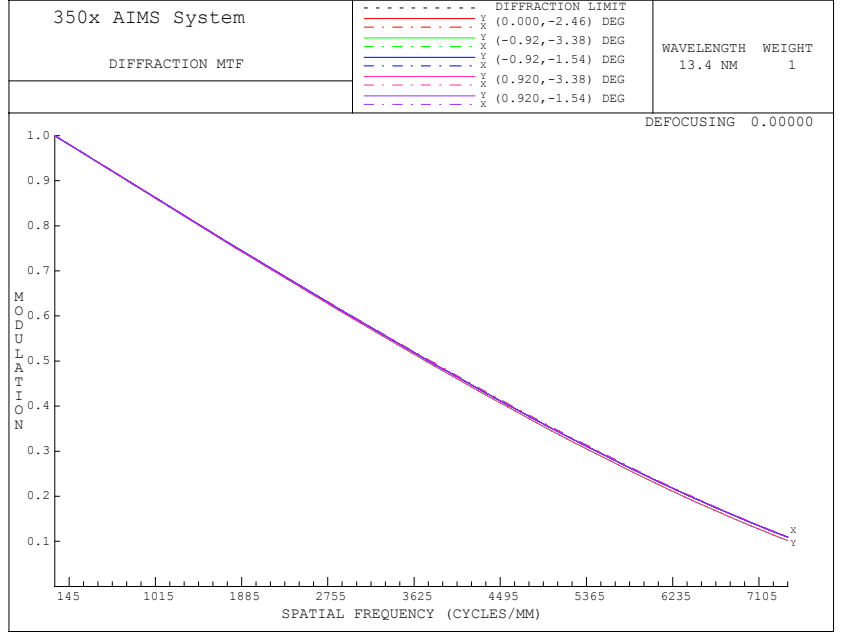

MTF response

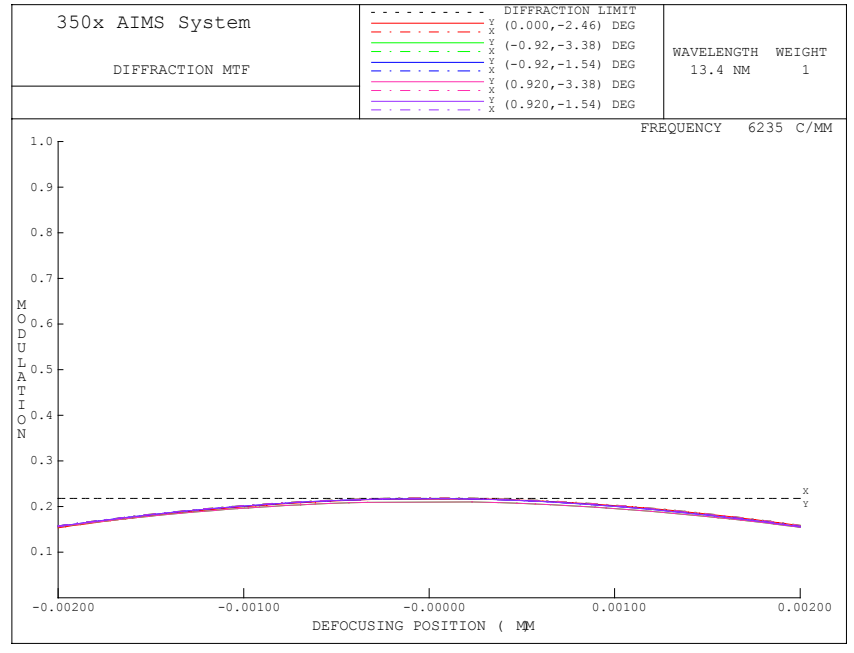

Contrast through focus at $62501 \mathrm{p} / \mathrm{mm}$

Figure 27

MTF and contrast response through focus for equal-radii AIM mode objective design demonstrating excellent performance across the field.

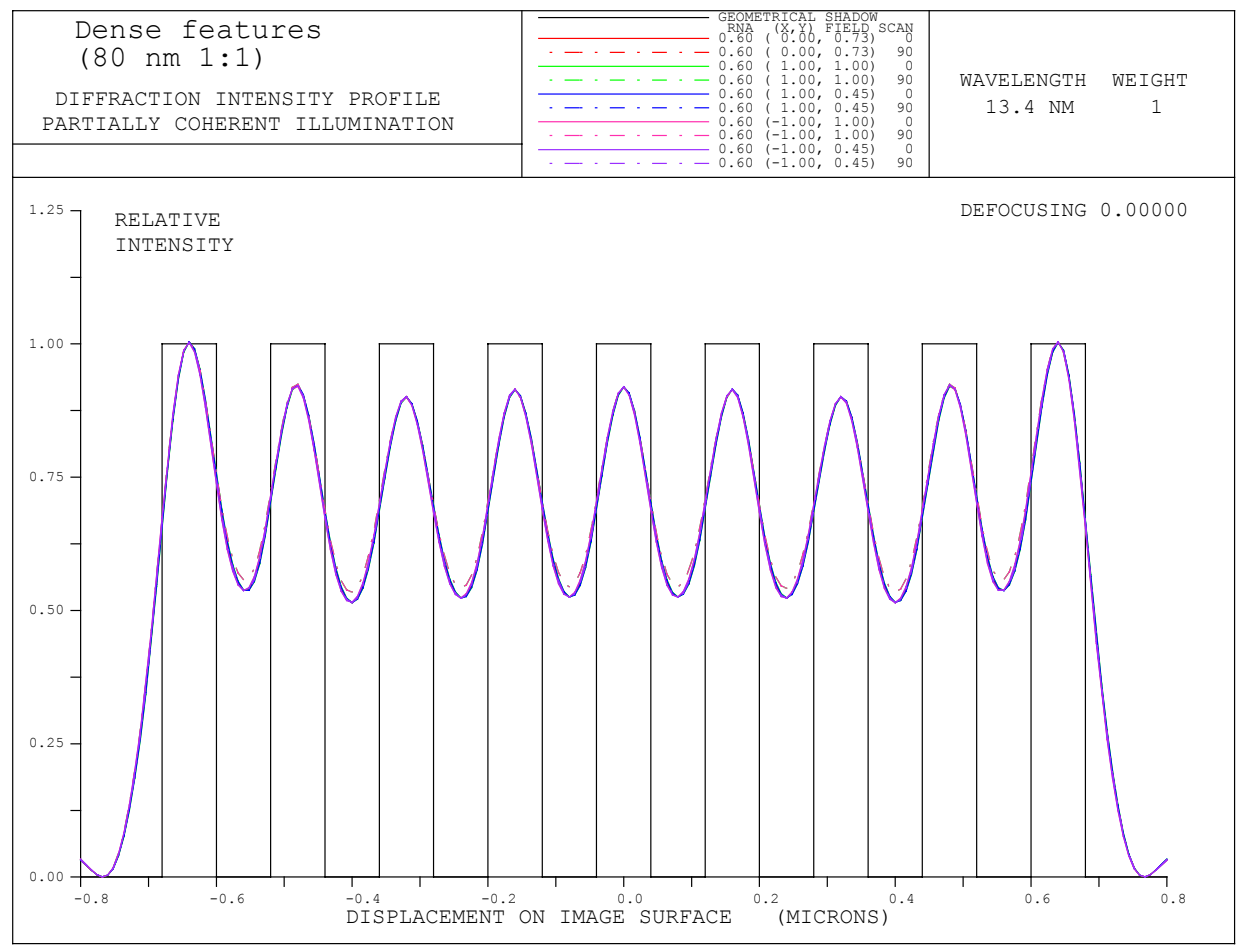

Figure 28

Partially coherent imaging at $6250 \mathrm{lp} / \mathrm{mm}$, corresponding to of $80 \mathrm{~nm}$ features at the mask, $\sigma=0.6$. 


\subsection{Summary of AIM objective configurations}

We summarise the comparative performance of different objective configurations in the table below. This table represents a summary of the options considered and can not describe a full system analysis, which is described in the preceding discussions; however it does highlight at a glance the relative strengths and weaknesses of each option considered so far. Inspection of this table reveals that the novel design discussed above is the most promising candidate for an AIM objective as it satisfies all the criteria of AIM mode imaging, thus it is the design we pursue through to a final system design.

\begin{tabular}{|c|c|c|c|c|c|c|c|c|}
\hline & $\begin{array}{l}3 \\
\frac{3}{5} \\
4 \\
0 \\
0 \\
0 \\
0 \\
0\end{array}$ & $\overleftrightarrow{Z}$ & 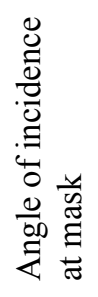 & 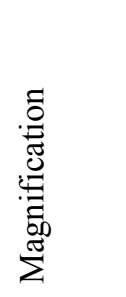 & 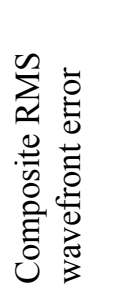 & 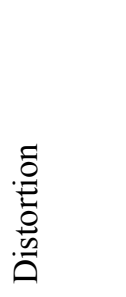 & 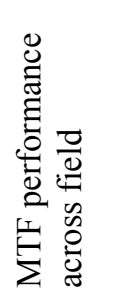 & 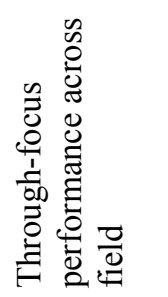 \\
\hline Spherical Schwarzschild & $50 \times 50 \mu \mathrm{m}$ & 0.0625 & $11.8^{\circ}$ & $10 \mathrm{x}$ & $55 \mathrm{~m} \lambda$ & $87 \mathrm{~nm}$ & Poor & Variable \\
\hline Modified Schwarzschild & $50 \times 50 \mu \mathrm{m}$ & 0.0625 & $7.5^{\circ}$ & $10-20 x$ & & & & \\
\hline Aspheric Schwarzschild & $50 \times 50 \mu \mathrm{m}$ & 0.0625 & $11.8^{\circ}$ & $10 \mathrm{x}$ & $0.6 \mathrm{~m} \lambda$ & $3 \mathrm{~nm}$ & Good & Good \\
\hline $\begin{array}{l}\text { Tilted Schwarzschild }\left(4^{\circ}\right) \\
\text { Parallel image plane }\end{array}$ & $50 \times 50 \mu \mathrm{m}$ & 0.0625 & $6^{\circ}$ & $10 \mathrm{x}$ & $66 \mathrm{~m} \lambda$ & $246 \mathrm{~nm}$ & Poor & $\begin{array}{c}\text { Poor and } \\
\text { variable }\end{array}$ \\
\hline $\begin{array}{l}\text { Tilted Schwarzschild }\left(4^{\circ}\right) \\
\text { Tilted image plane }\end{array}$ & $50 \times 50 \mu \mathrm{m}$ & 0.0625 & $6^{\circ}$ & $10 \mathrm{x}$ & $1.9 \mathrm{~m} \lambda$ & $5833 \mathrm{~nm}$ & Good & $\begin{array}{c}\text { Poor and } \\
\text { variable }\end{array}$ \\
\hline Modified MET & $600 \times 200 \mu \mathrm{m}$ & 0.0625 & $7^{\circ}$ & $5 x$ & $21 \mathrm{~m} \lambda$ & $2.2 \mathrm{~nm}$ & Good & Good \\
\hline Novel AIM design & $50 \times 50 \mu \mathrm{m}$ & 0.0625 & $6^{\circ}$ & $5-20 x$ & $0.6 \mathrm{~m} \lambda$ & $3 \mathrm{~nm}$ & Good & Good \\
\hline
\end{tabular}

Table 2

Summary of performance characteristics of candidate AIM-mode objective configurations studied.

\subsection{Diffractive optical elements}

The above discussion has focussed on systems using normal-incidence multilayer coated optics, however it is also possible to use diffractive lenses, or zone plates, for EUV imaging. There may be a significant cost advantage in following this approach as a set of zone plates may cost on the order of $\$ 5,000$ to fabricate using established E-beam lithography techniques, significantly less than the anticipated cost of fabricating and coating normal-incidence multilayer optics. However, there are a number of issues particular to diffractive optics which must be addressed in order to evaluate the feasibility of this approach. In particular issues that need particular attention for a zone plate system include bandwidth and its effect on the aerial image, working distance to the mask, zone placement errors and their effect on flare and contrast transfer, keystone distortion and field curvature.

Although we do not choose to pursue a zone plate design for the purposes of this study, in fairness to vendors who may propose a zone plate design and so as to provide a more comprehensive analysis we provide additional discussion on issues related to a zone plate design in the preliminary system design section later in this report. 


\section{Potential illuminator designs}

Having assessed potential objective configurations, it is also necessary to consider condenser designs capable of illuminating the mask from a compact plasma source. Although several objective configurations were studied in section 3, in this section we limit the scope of illuminator designs to configurations suitable for use with the novel AIM objective configuration identified in section 3 for further analysis and system integration within the scope of this study.

\subsection{Illuminator configurations for AIM mode}

The AIM-mode objective is an on-axis design with mild aspheres and is fabricated from a single rotationally symmetric parent optic. Only one half of this optic is used for the AIM mode imaging, leaving the other half of the objective available for introducing the illumination, in principle enabling both illumination and imaging up to the full $0.0625 \mathrm{NA}$ at $6^{\circ}$ angle of incidence. In order to introduce illumination into the objective without obscuring the imaging rays a series of grazing incidence fold mirrors can be used as shown in Figure 29.

To provide Köhler illumination the primary collector mirror $\mathrm{C} 1$ is imaged onto the mask via an intermediate image located between $\mathrm{C} 2$ and the fold mirrors. An aperture stop could be located in this plane to control the size of the illuminated field, whilst an aperture stop at either $\mathrm{C} 2$ in the condenser or $\mathrm{M} 2$ in the objective could be used to alter the pupil fill pattern. Variation in pupil fill pattern may be achieved by altering the size and shape of the illuminator pupil stop, although this flexibility comes at the expense of a loss of throughput as the custom fills are obtained by blocking unwanted illumination, thereby reducing the flux transmitted through the condenser chain. Note also that in this design the illuminator condensers are located some distance from the mask and imaging optics, which aids in debris mitigation from the EUV source. If required, a transmissive spectral purity filter could be placed in the condenser path to isolate the mask and imaging optics from the illuminator chamber.

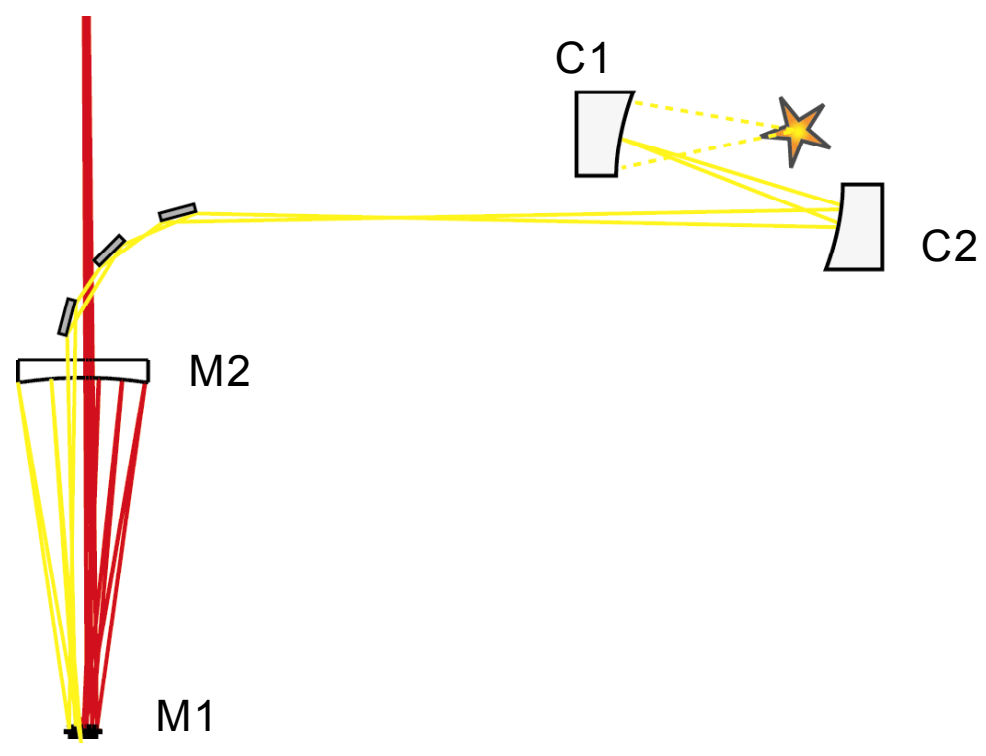

Figure 29

Köhler condenser implementation for EUV AIM-mode objective

\section{2 Étendue limitations on condenser collection efficiency}

Conservation of energy and geometrical optics dictates that the energy transmitted per unit time through any crosssection of a tube of rays is constant. In particular if a bundle of rays intersects a constant $\mathrm{z}$ plane over an infinitessimal of area $d x d y$ and has a small range of angles $d \alpha d \beta$ then as the light propagates though an ideal, lossless system the quantity

$$
n^{2} B(x, y ; \alpha, \beta) d x d y d \alpha d \beta=n^{2} B(x, y ; \alpha, \beta) d A \cos \theta d \omega
$$

is conserved, where $d A=d x d y$ is a differential unit of area, $d \omega$ is the solid angle, $\theta$ is a measure of the angle of the ray bundle relative to the normal of the plane of integration, $n$ is the index of refraction and $B(x, y ; \alpha, \beta)$ is the photometric brightness at the point $(x, y)$ in the direction $(\alpha, \beta)$ and measures the time-averaged amount of light 
energy travelling in a given direction at a given point. In condenser design, the integral of the brightness $B(x, y ; \alpha, \beta)$ over all angles determines the amount of energy per unit area incident on the object, and the integral over all angles and positions determines the amount of energy delivered to the object by the condenser. Specifically, the integral

$$
\int n^{2} B(x, y ; \alpha, \beta) d x d y d \alpha d \beta=\int n^{2} B(x, y ; \alpha, \beta) d A \cos \theta d \omega
$$

is the étendue or geometric extent of an extended object and is also conserved when propagated through a loss-less system. If $B$ is independent of direction the radiation is said to be isotropic and, if the radiating surface is a plane, the photometric intensity in any direction then varies as the cosine of the angle to the surface normal and the source is said to be Lambertian.

The total amount of power from a Lambertian object that can be transferred through a perfect lens is

$$
\frac{1}{\pi} M \int_{\text {field }} d x d y \int_{\text {pupil }} d \alpha d \beta
$$

which is referred to as the total lens étendue and, if the pupil is circular with constant illumination over the field, it can be shown that the étendue is porportional to $A(N A)^{2}$ where A is the area of the field and $N A$ is the numerical aperture of the lens. This relationship holds for a perfect imaging or condenser system and therefore places an upper bound on the amount of power that can be transmitted through a given optical system with a given field size and NA. An actual condenser will in general not have perfect transmittance thus this relationship places an upper bound on the possible illumination - actual throughput will depend on the particular condenser design.

In the context of the design of a mask inspection microscope, the pupil of the imaging optics will be $0.0625 \mathrm{NA}$ and the field of view will be on the order of $50 \mu \mathrm{m}$ square. Conservation of étendue then gives the following upper bound on the condenser design

$$
\begin{aligned}
A_{\text {source }}\left(N A_{\text {condenser }}\right)^{2} & =A_{\text {mask }}(\sigma N A)^{2} \\
& \approx(50 \mu \mathrm{m})^{2}(0.0625 \sigma)^{2}
\end{aligned}
$$

The coherence factor $\sigma$ of the illumination ${ }^{13}$ for a lithographic system will typically be less than unity, $\sigma<1$, and the source size is determined by vendor specifications, typically of the order of $200-500 \mu \mathrm{m}$ in diameter for gas plasma sources and as small as $20 \mu \mathrm{m}$ in diameter for low power solid target sources. This immediately tells us that we will either have to collect a small NA of a large source, with the consequent inefficiencies due to a small collection solid angle, or use a smaller source in order to be able to collect a larger fraction of the light. This source selection design trade-off will be discussed in more detail later when formulating the throughput budget for the AIM tool.

\subsection{Illumination and objective configurations for high-resolution mode}

For AIM mode imaging we have taken great care to ensure that the angles of incidence, NA, wavelength, bandwidth and ultimately imaging performance mimic the salient imaging properties of a production stepper system. Whilst necessary for AIM mode, in high resolution mode there is no requirement that the imaging emulate the performance of a stepper, thus there is more flexibility and a wider range of potential objective designs for highresolution mode. At the same time there is no ready metric of performance against which to measure high-resolution objective performance, thus it is harder to dismiss alternative options and narrow down the field of candidate highresolution systems. Looking to solutions with the lowest cost path we investigate high-resolution implementations that reuse the AIM mode objective described above in a different configuration for high-resolution imaging ${ }^{14}$. An alternative approach could involve the design of a dedicated high-resolution objective that can be interchanged with an AIM objective leaving the rest of the integrated system untouched.

\footnotetext{
13 The coherence factor $\sigma$ of the illumination is the ratio of the NA of the illumination to the imaging optics, $\sigma=N A_{\text {illum }} / N A_{\text {imaging }}$. Incoherent illumination corresponds to $\sigma=\infty$.

${ }^{14}$ It is always possible to design a dedicated high-resolution objective that can be interchanged with an AIM objective leaving the rest of the integrated system untouched. For example, a high-NA, higher magnification Schwarzschild objective could be interchanged with the AIM objective to enable high-resolution mode.
} 


\subsubsection{AIM mode objective adapted for high-resolution mode}

There is an attraction to using the same objective for both AIM and high-resolution mode imaging, so we seek ways in which to adapt the objective identified above as the most promising design for AIM mode imaging for use at higher resolution. This can be done by allowing light collection over the full pupil, rather than just the $0.0625 \mathrm{NA}$ subaperture at $6^{\circ}$ to the normal as in AIM mode.

Opening up the aperture and departing from the AIM condition, as shown in Figure 30, enables the full 0.166NA parent aperture (minus some central obscuration) to be collected and projected to the intermediate image plane. Because of clearance constraints from the M2 mirror it is not possible to collect more than 0.166NA using this objective design; at $0.166 \mathrm{NA}$ the diffraction-limited spatial resolution is $40 \mathrm{~nm}$ or almost 2.5 times the spatial resolution of the AIM mode objective, thus operating the objective in this mode does enable higher resolution imaging than in AIM mode. The problem then becomes one of illuminating the mask to take advantage of this full aperture, and solutions present themselves for use in both brightfield and a darkfield imaging modes.

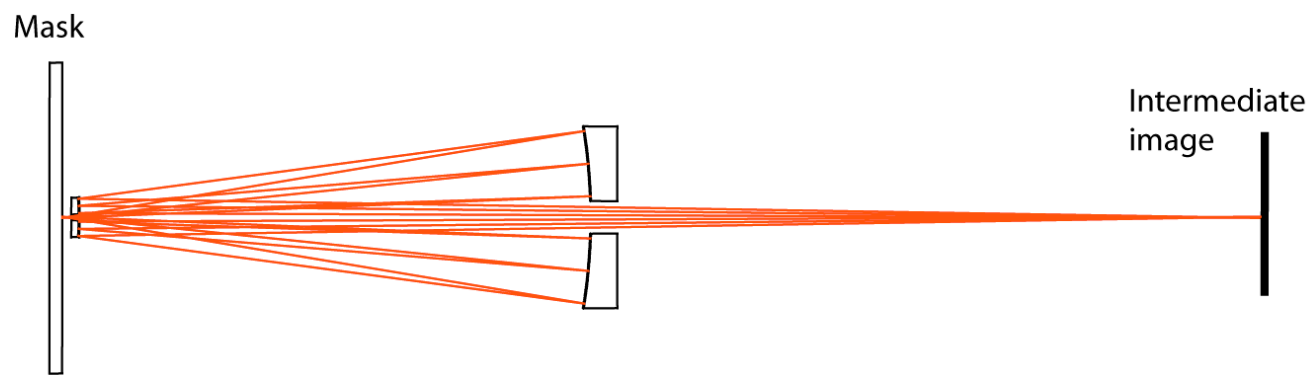

Figure 30

AIM mode objective used in high-resolution mode. Imaging takes place using the full $0.166 \mathrm{NA}$ parent aperture enabling higherresolution imaging than in AIM mode.

\subsubsection{Illumination for bright-field imaging}

In brightfield mode the illumination is contained in the clear aperture of the imaging system so that the specular reflection components are collected by the objective. To enable full pupil illumination in this mode for a reflective object it is necessary to use a beamsplitter in the illumination chain as shown in Figure 31 below. This enables the full pupil to be filled if desired, but comes at the expense of discarding one polarisation and having at best $25 \%$ throughput (two passes through the beamsplitter, 50\% loss on each path). The fabrication of EUV beamsplitters by depositing mutilayer mirrors with 7 or so periods on a SiNi membrane has been demonstrated to be feasible. The imaging properties of these beamsplitters has not been extensively studies, however we note that the imaging path of the beamsplitter is used in transmission thus only thickness variations will be translated into phase errors. Finish and flatness errors will map into aberrations of the illumination beam, which is in general less critical than aberrations in the imaging path.

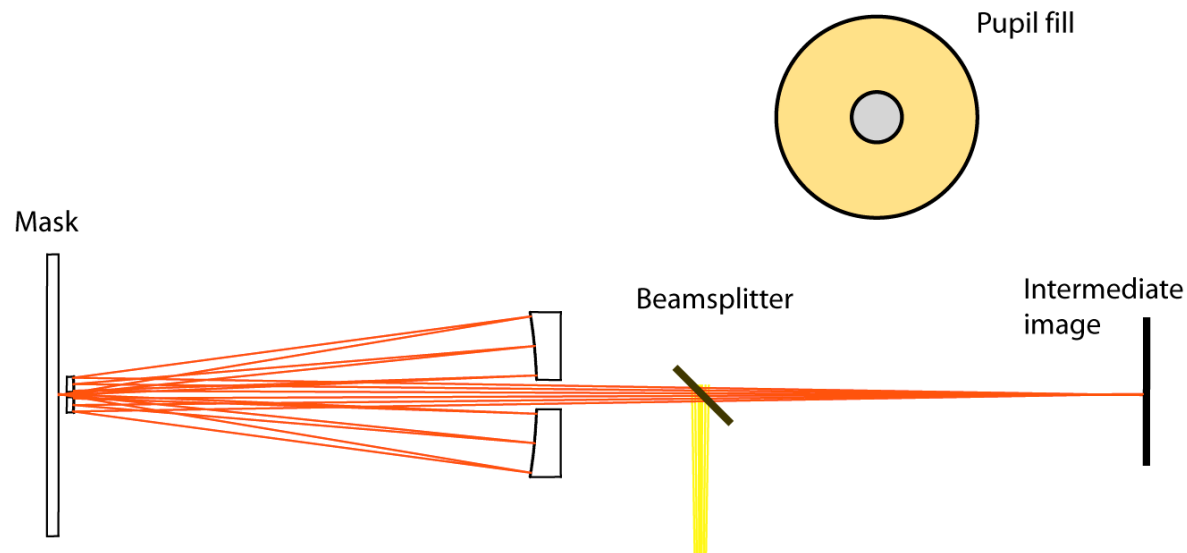

From condenser

Figure 31

Insertion of illumination for bright-field mode using either a beamsplitter. The full pupil is illuminated, but one polarisation is dropped using the $45^{\circ}$ mirror and only $25 \%$ of what remains passes through to the image plane (50\% loss on each pass through the beamsplitter). 
An alternative to using full pupil fill with a beamsplitter is to use an annular pupil fill, as shown in Figure 32. This type of illumination could be achieved by depositing a small annular beamspliter on a SiNi membrane and has the advantage that the beamsplitter occupies only a small proportion of the total pupil.

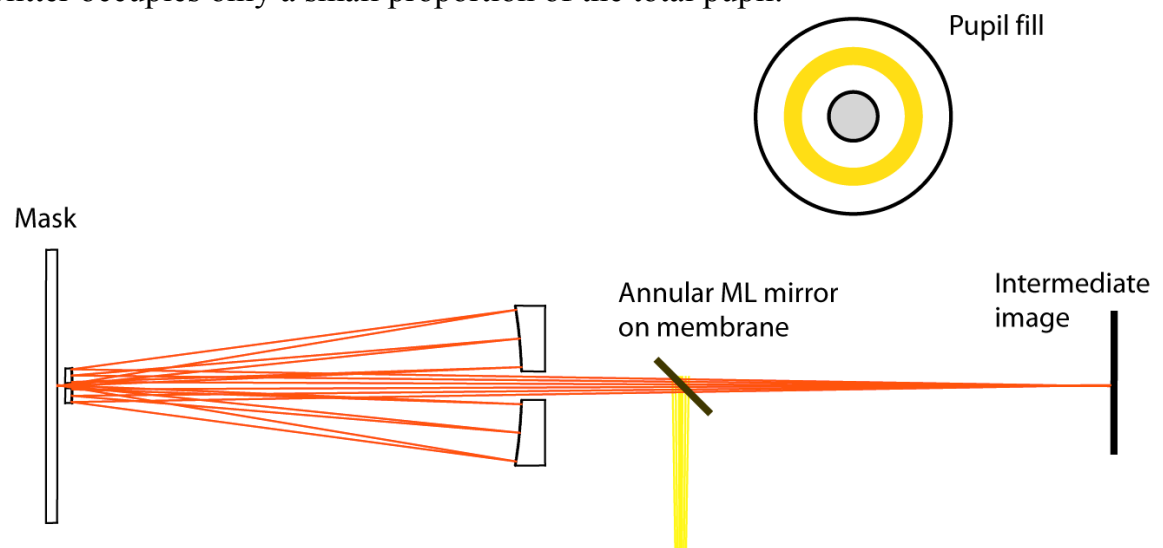

From condenser

Figure 32

Insertion of illumination for bright-field mode using either a beamsplitter. The full pupil is illuminated, but one polarisation is dropped using the $45^{\circ}$ mirror and only $25 \%$ of what remains passes through to the image plane (50\% loss on each pass through the beamsplitter).

\subsubsection{Dark-field imaging}

In dark-field imaging the specular reflection component is blocked from passing back into the objective, leaving only the scattered light to pass through the imaging system. This highlights the high spatial frequency information, displaying clearly the edges of objects at the expense of discarding the low frequency components, and could be implemented on the AIM mode objective as shown in Figure 33.

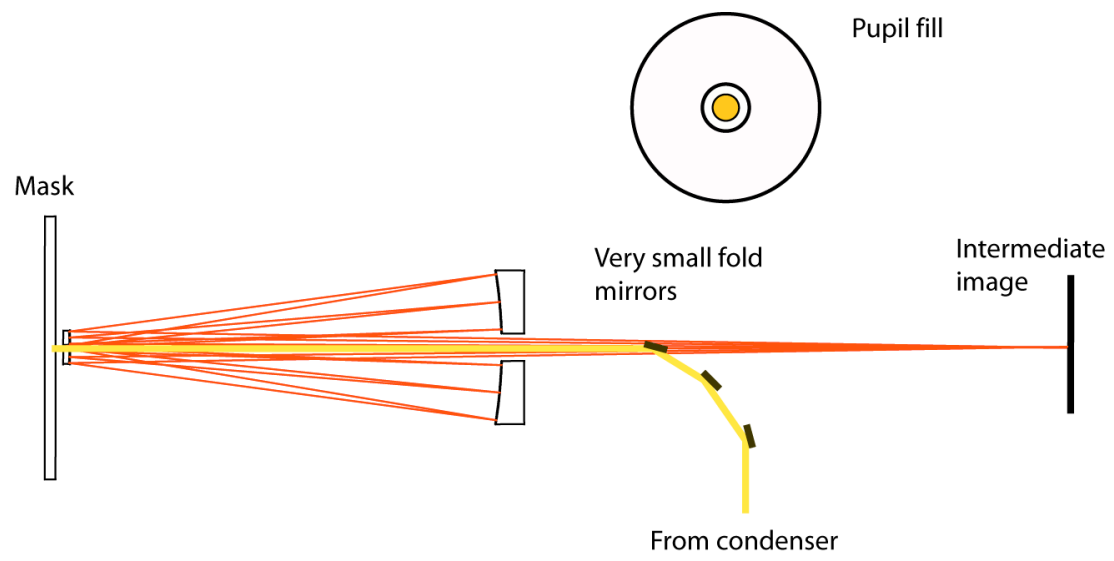

Figure 33

Illumination for dark-field mode using a narrow illumination pencil transmitted through the central aperture in M1 and M2. The zero-order reflection returns along the illumination path so that only light scattered into the imaging pupil passes to the image plane.

It is instructive to compare the aerial images obtained with these high-resolution implementations with the performance expected in AIM mode to demonstrate that high resolution information useful for effecting pattern repair is collected using the above objective configurations. A sample of high-resolution aerial images are shown in Figure 34 below illustrating the anticipated capability of the high-resolution imaging and illumination schemes described above. 


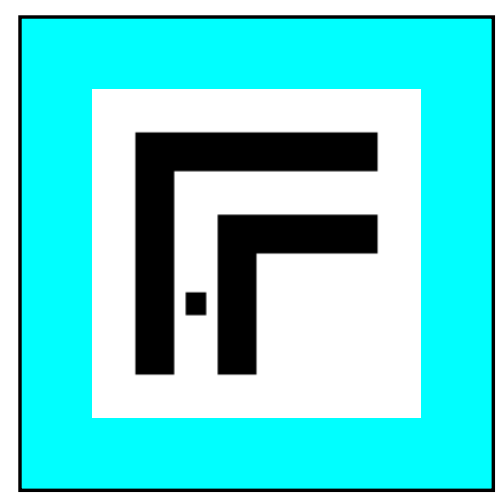

Test object $-45 \mathrm{~nm}$ lines with a proximity amplitude defect located between the lines
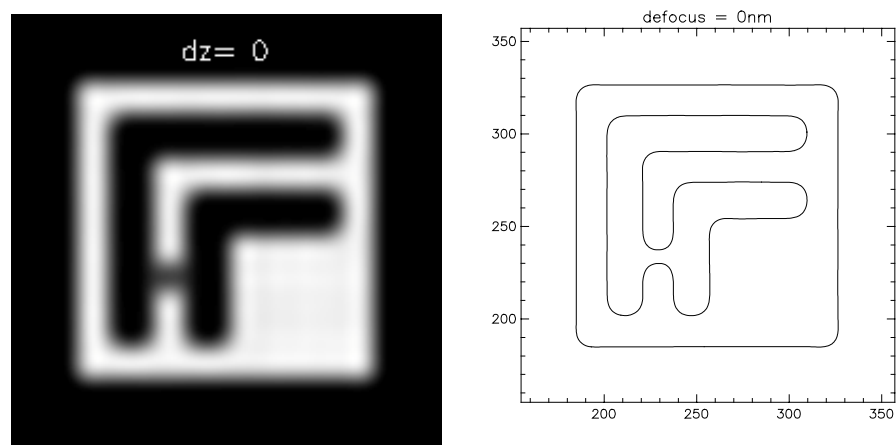

Aerial image and $40 \%$ threshold resist model obtained with AIM mode imaging
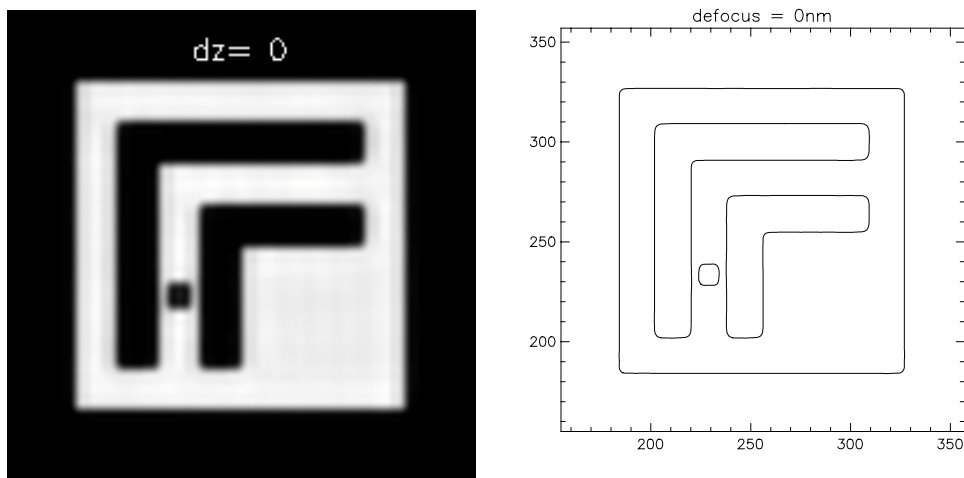

Aerial image and $40 \%$ threshold using a beamsplitter to introduce illumination
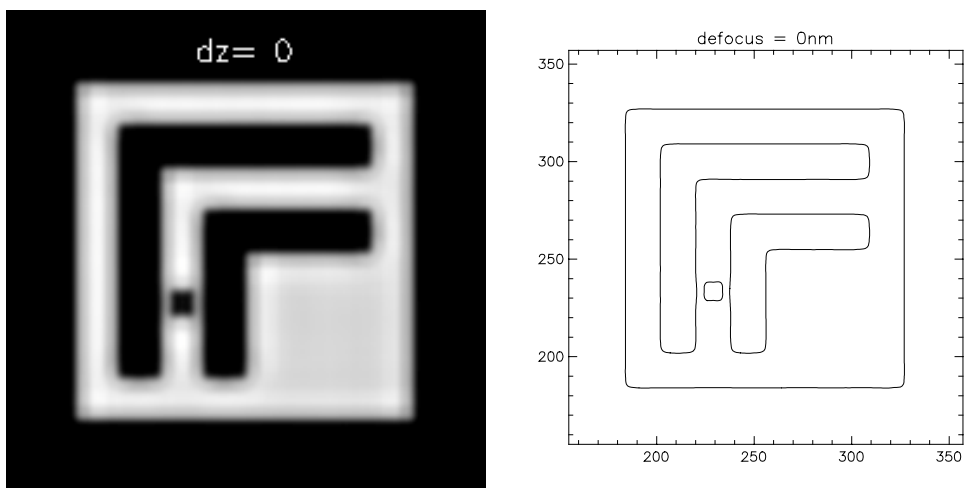

Aerial image and $40 \%$ threshold using annular illumination

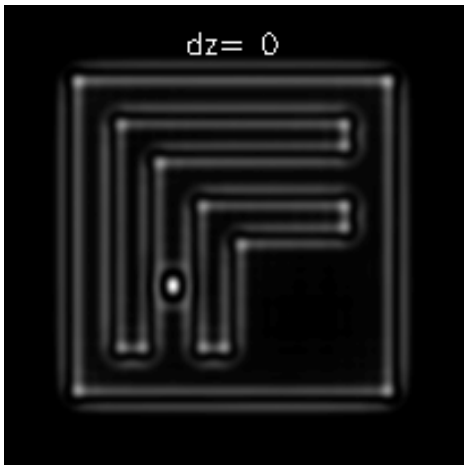

Aerial image obtained in dark-field mode. Maximum intensity in the darkfield image is 0.96 .

Figure 34

Aerial image examples of a test pattern obtained in AIM mode (top) compared to the aerial image in high-resolution mode. The increase in spatial resolution is evident. 


\section{$5 \quad$ Integrated system design and analysis}

We now proceed to complete preliminary system designs for an EUV AIM system based on three potential platforms. As mentioned in the introduction we consider three systems:

- a system based on a visible light microscope

- An electron microscope based system; and

- An all-EUV system consisting of normal-incidence multilayer coated mirrors

Each of these potential configurations are discussed in turn below.

\subsection{Visible light microscope based system}

Adapting a visible light microscope for use in an EUV AIM system has attraction as it leverages the experience gained by existing $193 \mathrm{~nm}$ and $156 \mathrm{~nm}$ AIM tool vendors in adapting visible light microscopes for use as an AIM tool. This can not be done directly with EUV as the optics have to be reflective and in vacuum, however use of a visible light microscope platform for secondary magnification is possible if a scintillator is used to convert an EUV image into visible light. We note two potential user benefits of adopting this approach over an all-EUV system: (1) From a user point of view there is minimal change in method and ergonomics compared to existing AIM tools, and (2) visible-light epi-illimination introduced from the microscope may provide sufficient illumination to view the mask directly using visible light, providing some capacity for parallel visible light and EUV inspection of the mask on the same platform.

A conceptual layout of how such a combined EUV/visible light AIM system could be implemented is shown below in Figure 35.

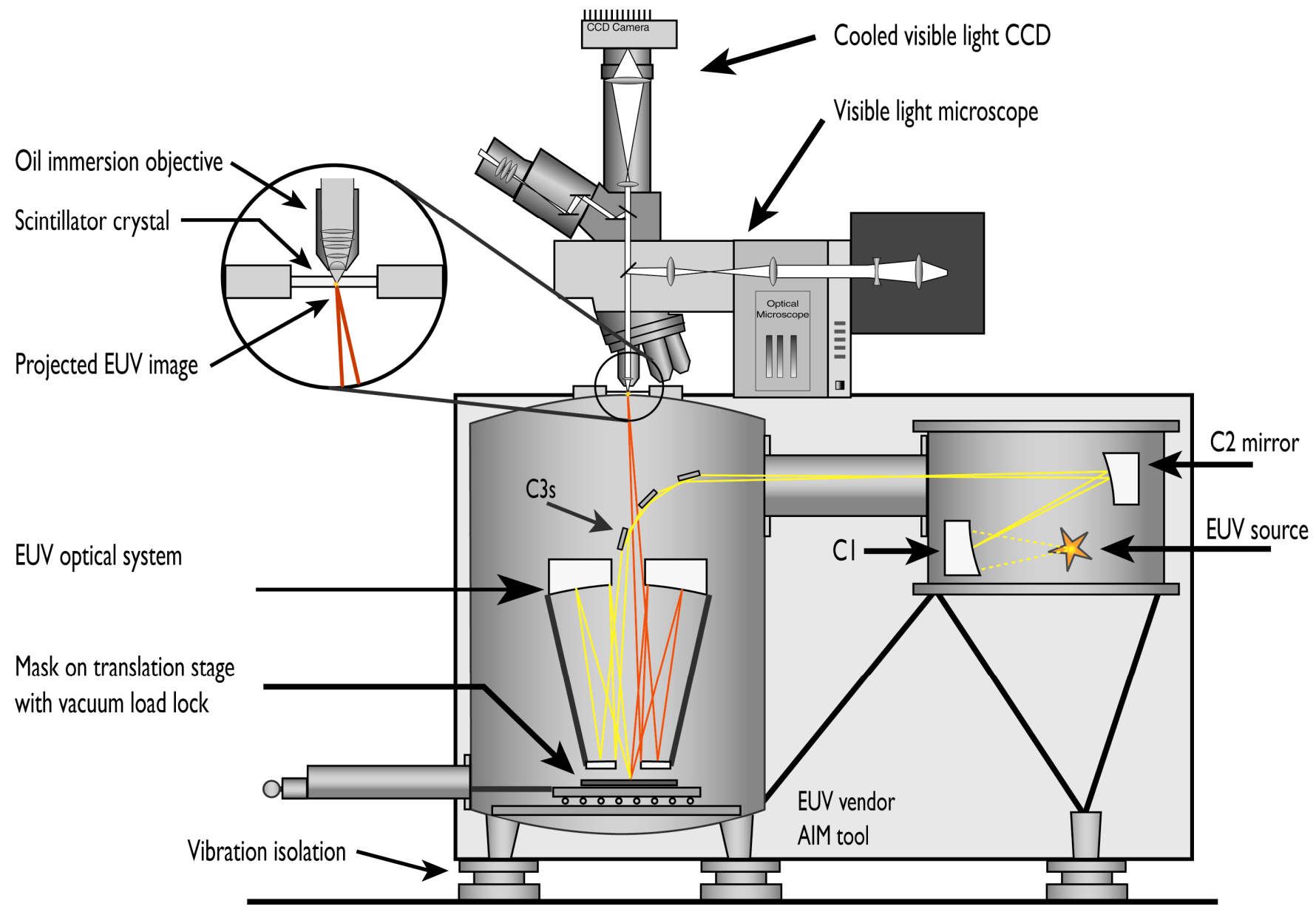

Figure 35

Conceptual layout of a combination EUV and visible light microscope system. 


\subsubsection{Critical subsystem description}

Key components specific to this system are:

- A scintillator to convert the 10x magnified EUV image into a visible light image

- A visible light microscope to provide magnification of the EUV image formed on the

- EUV optics of approx 10x magnification to image the mask onto the scintillator

\subsubsection{Visible light microscope, microscope objective and CCD}

A visible light microscope is required to provide additional magnification after the scintillator. There are many commercial manufacturers of high-quality microscopes and microscope objectives from which this component could be sourced: for example Leica, Nikon, Olympus and Zeiss, thus obtaining this component poses no problem.

In order to maximise both spatial resolution and collection of fluorescent light it is desirable to use a highnumerical aperture objective, preferably an oil immersion objective to minimise refractive losses in passing from the scintillator to air. Objectives designed to maximise collected light are manufactured for use in biological fluorescence microscopy and the use of such a lens is suggested. Biological objectives are typically corrected to compensate for the spherical aberration introduced when imaging through a $0.17 \mathrm{~mm}$ thick cover glass, thus the scintillator thickness can be chosen to have the same optical thickness as a cover glass ensuring aberration-corrected imaging into the scintillator. Furthermore the penetration depth of EUV into the scintillator is approximately $20 \mathrm{~nm}$, thus all of the fluorescence will be within the depth of focus of the objective ensuring maximum resolution and imaging quality. An example of this type of objective is the Zeiss Plan-Neofluar 40x 1.3NA oil immersion objective which has a $0.2 \mathrm{~mm}$ working distance and is designed for use with a $0.17 \mathrm{~mm}$ thick cover slip, although similar objectives are manufactured by other vendors would also be suited for the task.

Magnification of the visible light microscope should be sufficient to enlarge the scintilator image onto the CCD over an acceptable field of view, but there is some freedom to select this depending on the reimaging lenses used to transfer the objective image to the CCD. Choice of supplier and specific objectives and magnifications is up to the vendor. High-quality visible light CCDs are also readily available from a number of vendors, for example Photometrics amongst others, and are known to be compatible with visible light microscopes and image processing software.

\subsubsection{Scintillator}

A key element component of this design is a scintillator to convert the EUV radiation to visible light, and this can be done either with phosphors or scintillator crystals. Phosphors have a higher conversion efficiency than scintillators, however the grain size of phosphors is typically $>2 \mu \mathrm{m}$ which limits the available spatial resolution that can be achieved in the converter, requiring a higher magnification in the EUV optics to overcome grain size effects. For maximum spatial resolution it is desirable to use transparent crystal scintillators in which the spatial resolution is limited by EUV penetration into the scintillator rather than grain size.

Scintillators of this type are commercially available and may be sourced from a variety of vendors, for example StarTech instruments (http://www.startechinst.com) and Crismatec (http://www.crismatec.com), and the final choice of supplier and actual scintillator specifications is up to the vendor provided adequate spatial resolution is maintained.

For the purposes of this design study we base our discussion on a scintillator made by StarTech instruments. We suggest using a $0.17 \mathrm{~mm}$ thick scintillator as this is the thickness of cover glass for which the spherical aberration of microscope objectives is generally corrected, and matching this thickness ensures the best imaging at least cost. This is a custom thickness and may take 8 weeks to deliver, however off-the-shelf thickness of between 100 and $500 \mu \mathrm{m}$ are available at a cost of approx. $\$ 130$ per unit for a $6 \mathrm{~mm}$ diameter scintillator.

The two technical issues which must be addressed are spatial reolution and conversion efficiency. The spatial resolution of a scintillator is approximately equal to the full angle of divergence of the input beam at the scintillator times the penetration depth of the radiation. For $13.5 \mathrm{~nm}$ EUV the penetration depth into the scintillator is very small typically $20 \mathrm{~nm}(1 / \mathrm{e})$. Thus for a $0.0625 \mathrm{NA}$ objective $(0.25 / 4$ AIM objective operating at $10 \mathrm{x})$ the radial bloom would be of the order of $0.2 \mathrm{~nm}$, increasing to $12 \mathrm{~nm}$ at $0.3 \mathrm{NA} 10 \mathrm{x}$ objective. Both of these figures are significantly less than the diffraction-limited resolution of a visible light microscope, thus we anticipate that bloom and scintillator resolution are is not a limiting factor in this approach.

No reliable data was found on the conversion efficiency of the scintillator at $100 \mathrm{eV}(13.5 \mathrm{~nm})$ so this quantity has to be estimated from figures available in the literature for similar materials ${ }^{15}$. The conversion efficiency has been

\footnotetext{
${ }^{15}$ B.LaFontaine et.al. "Submicron soft X-ray fluorescence imaging" (1995) 66(3) Appl.Phys.Lett. 282. The solid angle into which this emission is transferred is not specifically stated so we assume it to be emission into $4 \pi$.
} 
estimated for one type of scintillator $\left(\left(\mathrm{Y}_{2-\mathrm{x}} \mathrm{Tb}_{\mathrm{x}}\right) \mathrm{SiO}_{5}\right)$ as being $6 \pm 4 \%$ energy yield, corresponding to $2.4530 \mathrm{~nm}$ photons out for every incident EUV photon ${ }^{16}$. Note that the same paper reports an overall energy transfer from the scintillator plane to the CCD in the vicinity of $0.25 \%$, the additional drop in efficiency being due to losses in the optical imaging system used for that experiment. The optical system could be further refined to maximise throughput thus we prefer to work with the measured energy yield rather than quoted system throughput. Measurement and verification of the conversion efficiency of the scintillator to be used is therefore an essential step in implementation of this design.

We also note that the conceptual drawing in Figure 35 shows the scintillator being used as a vacuum window. This has certain advantages for system integration in that all the visible light optics are in air and an oil immersion objective can be used to maximise light collection. The vacuum need only be supported across a small area corresponding to the field of view of the objective, which is less than $1 \mathrm{~mm}$ in diameter. This would appear to be practical but would require demonstration. An alternative approach would be to locate both the scintillator and objective within the vacuum chamber. This has an advantage from a structural point of view in that the thin scintillator is not supporting the vacuum, but requires imaging through a vacuum viewport, use of a vacuum compatible microscope objective and does not allow use of oil immersion to maximise collection of the fluorescence. If the EUV/visible system is pursued by a vendor the selection of which approach to follow will have to be made by the vendor as part of an overall risk and cost/benefit analysis.

\subsubsection{EUV projection optics}

There is naturally flexibility in choice of magnification and it is open to a vendor to choose the magnification for the EUV stage. To guide the customer in assessing various proposals, we note that a 1.3NA objective imaging visible-band fluorescence in the region of $500 \mathrm{~nm}$ will have incoherent diffraction-limited spatial resolution of

$$
\delta x=\frac{\lambda}{2 N A}=192 \mathrm{~nm} .
$$

At the very least we would require each pixel in the mask plane to be magnified to this size in order to ensure adequate sampling of the optical image, which with 44nm mask-plane pixels necessitates an EUV magnification of at least 4x. Remembering that the MTF of the whole system will be the product of the MTF of the microscope and EUV optics, and that the visible light optics imposes an additional high-frequency cutoff, we would suggest generous oversampling in the visible light stage so as to minimise image degradation in the visible light portion of the system, suggesting a higher magnification system. On the other hand increased EUV magnification reduces the available field of view, and as an upper bound there is no point magnifying the image with EUV optics so much that no advantage is gained over direct detection by a soft X-ray CCD with $13.5 \mu \mathrm{m}$ pixels.

A working magnification of 10x for the EUV optics gives a good balance of spatial resolution and field of view. This magnifies each $89 \mathrm{mn}$ mask-plane resolution element to $890 \mathrm{~nm}$ in the scintillator plane, which is well within the microscope resolution limit. If the scintillator is imaged with 40x optics onto a $1024 x 1024 \mathrm{CCD}$ with $6.8 \mu \mathrm{m}$ pixels each CCD pixel corresponds to $170 \mathrm{~nm}$ in the scintillator plane over a field of $200 \mu \mathrm{m}$, translating into $17 \mathrm{~nm}$ pixels over a field of $20 \mu \mathrm{m}$ in the mask plane. This gives an effective 5.3 pixels per mask resolution element, sufficient to amply sample the mask plane image.

We turn now to the design of a suitable EUV objective. As mentioned above there is flexibility in the design and selection of magnification for the EUV optics stage, and it is open to the vendor to make choices on this subject to the requirement that the imaging is faithful to the imaging properties of a 6-mirror stepper system. At first glance a Schwarzschild-type system appears attractive, however the strict Schwarzschild condition leads to an angle of incidence of $11.8^{\circ}$ at the mask, whilst Schwarzschild-type designs give an angle of incidence of $7.5^{\circ}$ at the mask. This leads to excessive bandwidth-shifting and shadowing in the image which is not meaningful for AIM mode imaging. Tilting the Schwarzschild potentially solves the angle of incidence problem, however this leads to the creation of excessive distortion and loss of contrast in the image. Furthermore tilting the mask with respect to the objective also tilts the image plane, and with the tilt of the image being the magnification times the tilt at the mask this rapidly leads to large tilts when operating at 10x magnification. The tilted object and image planes in turn lead to an significant change in magnification across the field, which is undesirable for an AIMS-type tool.

We are therefore led to explore designs of the same family as the MET optics designed specifically for use in an AIM application. Employing this strategy we can design an optic with moderate asphericity which supports AIM imaging $6^{\circ}$ angle of incidence to the mask with a $0.0625 \mathrm{NA}$ clear aperture and 10x magnification with a manageable track length of $600 \mathrm{~mm}$. The performance properties of this system were discussed in detail above so we do not repeat them here.

\footnotetext{
${ }^{16}$ Ibid. The solid angle into which this emission is transferred is not specifically stated so we assume it to be emission into $4 \pi$.
} 


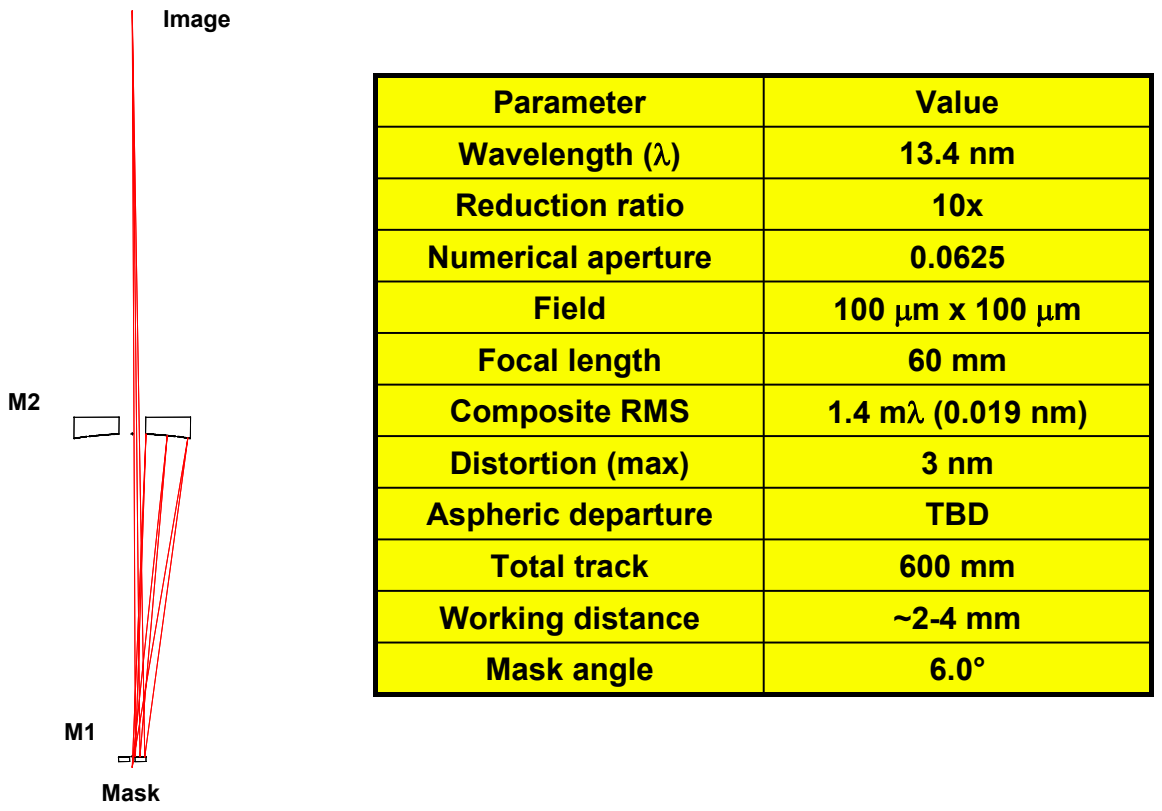

Figure 36

MET-type objective for 10x EUV magnification system.

\subsubsection{Matching magnification through the system}

To ensure that the EUV image is faithfully replicated through the visible light optics it is necessary to select a sufficiently high magnification for the EUV optics so that the resolution of the scintillator and visible light microscope are not limiting factors. One example of how magnification could be matched through the system is illustrated in Figure 37 below. Note that this is just one example of many possible combinations, and it is open to a vendor to select some other combination to suit their particular design.

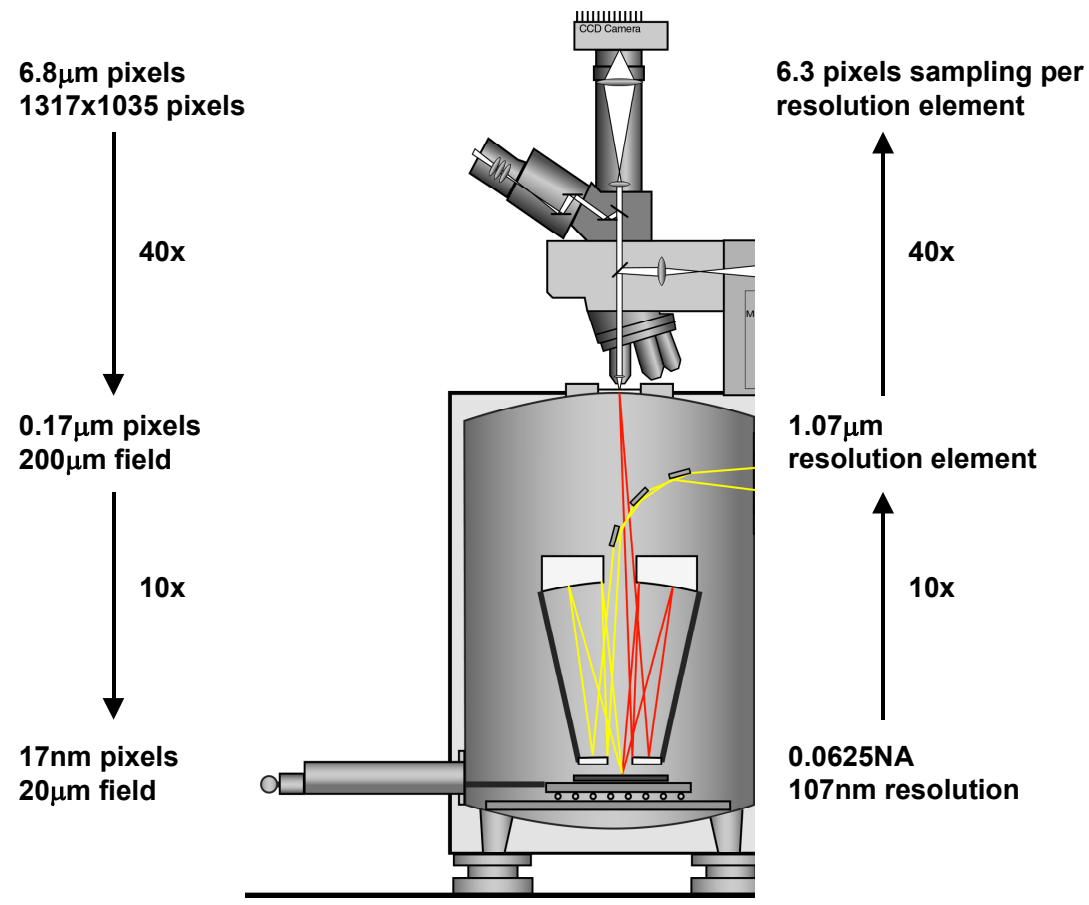

Figure 37

One possible combination of system magnifications designed to match spatial resolution into the visible light microscope. Vendors are free to use other combinations as part of an overall system trade-off. 
In this example we use a 10x EUV objective. Operating in AIM mode this objective has 107nm resolution at the mask, thus each objective resolution element is magnified to $1.07 \mu \mathrm{m}$ in size, well within the spatial resolution of the scintillator. Using 40x optical magnification, each mask resolution element gets magnified to 6 pixels in the CCD plane, ensuring adequate sampling of the image. Working from the CCD backwards, for 40x visible light magnification, each CCD pixel corresponds to $0.17 \mu \mathrm{m}$ in the scintillator plane, ensuring that the microscope PSF for a 1.4NA objective is adequately sampled. For a $1024 \times 1024$ CCD this gives a field of view of $20 \times 20 \mu \mathrm{m}$ at the mask, which could be easily increased using a larger CCD provided the microscope objective supports the larger field of view.

\subsubsection{Preliminary throughput budget}

We now proceed to calculate a preliminary throughput budget for a combination EUV/visible light system. Note that the figures in this throughput budget represent the best estimate based on information to hand at the time of writing. Individual vendors may choose different values for certain parameters that will flow through the throughput budget once changed. For example a vendor may have a different estimate of anticipated multilayer mirror reflectivity, collection efficiency, CCD parameters or magnification, and once changed these figures will flow through the spreadsheet and affect the final numbers.

In order to determine the required source power, and to ensure consistency between different approaches, we aim for a noise level of $1 \%$ in the detected image. In this regard it is important to point out that the source powers here are indicative only and it may well be feasible to use a smaller source, although exposure times will have to be lengthened, a number of frames integrated, or the allowed noise level increased to compensate for the lower EUV flux.

\begin{tabular}{|c|c|}
\hline \multicolumn{2}{|l|}{ Visible light conversion system } \\
\hline & \\
\hline & Value \\
\hline \multicolumn{2}{|l|}{ Target values } \\
\hline Noise level & $1 \%$ \\
\hline Exposure time & $1 \mathrm{sec}$ \\
\hline \multicolumn{2}{|l|}{ Visible light CCD } \\
\hline Total system noise (total shot noise) & $1.01 \%$ \\
\hline CCD type & KAF-1400 \\
\hline Pixel size & $6.8 \mathrm{um}$ \\
\hline Electrons per full well & 40,000 \\
\hline Measured number of electrons & 20,000 \\
\hline Array size & $1317 \times 1035$ \\
\hline Number of pixels (total) & $1,363,095$ \\
\hline electrons produced per incident photon & 1.00 \\
\hline Quantum efficiency & $60 \%$ \\
\hline Visible photons per pixel required for a full well & 33,333 \\
\hline \multicolumn{2}{|l|}{ Microscope system } \\
\hline Microscope magnification & $40 x$ \\
\hline Transmission efficiency & $90 \%$ \\
\hline Collected NA & 0.95 \\
\hline Collection angle & $71.82 \mathrm{deg}$ \\
\hline Fraction of $2 \mathrm{Pi}$ collected & $68.8 \%$ \\
\hline Visible light collection efficiency (out of $2 \mathrm{Pi}$ ) & $61.9 \%$ \\
\hline Visible light collection efficiency (out of $4 \mathrm{Pi}$ ) & $30.9 \%$ \\
\hline \multicolumn{2}{|l|}{ Scintillator crystal } \\
\hline CCD pixel size at scintillator & 0.17 um \\
\hline Field of view on scintillator (square) & 198 um \\
\hline Visible photons per EUV photon into $4 \mathrm{Pi}$ & 2.3 \\
\hline Energy conversion yield & $6.0 \%$ \\
\hline Number of visible photons emitted per pixel & 107705 \\
\hline Number of incident EUV photons needed per pixel & 46828 \\
\hline Incident EUV energy required per pixel & $4.68 \mathrm{E}+06 \mathrm{eV}$ \\
\hline EUV energy incident on scintillator (over field) & $6.38 \mathrm{E}+12 \mathrm{eV}$ \\
\hline
\end{tabular}




\begin{tabular}{|c|c|}
\hline EUV energy incident on scintillator (over field) & $1.02 \mathrm{uJ}$ \\
\hline \multicolumn{2}{|l|}{ Projection optics } \\
\hline Magnification & $10 x$ \\
\hline Collected NA & 0.0625 \\
\hline Nominal resolution & $107 \mathrm{~nm}$ \\
\hline PO box resolution unit size on scintillator & $1.07 \mathrm{um}$ \\
\hline Mirror reflectivity & $70 \%$ \\
\hline Number of reflections & 2 \\
\hline Wavelength matching factor & $99 \%$ \\
\hline Projection optic throughput & $49 \%$ \\
\hline \multicolumn{2}{|l|}{ Mask } \\
\hline CCD pixel size at mask & $17 \mathrm{~nm}$ \\
\hline Pixels per resolution element (sampling) & 6.3 \\
\hline Field of view (square) & 19.85 um \\
\hline Reflectivity of mask & $65 \%$ \\
\hline Wavelength matching factor & $98 \%$ \\
\hline Mask throughput & $64 \%$ \\
\hline Energy incident on mask over field per exposure & $3.31 \mathrm{uJ}$ \\
\hline \multicolumn{2}{|l|}{ System loss factors } \\
\hline Spectral purity filter throughput & $35 \%$ \\
\hline Transmission through gas & $50 \%$ \\
\hline Transmission through debris shield & $80 \%$ \\
\hline Throughput contribution of system loss factors & $14 \%$ \\
\hline \multicolumn{2}{|l|}{ Condenser } \\
\hline Coherence (sigma) & 0.8 \\
\hline Mirror reflectivity - normal incidence & $70 \%$ \\
\hline Mirror reflectivity - 45 degree & $32 \%$ \\
\hline Mirror reflectivity - grazing incidence & $90 \%$ \\
\hline Number of normal incidence reflections & 1 \\
\hline Number of 45 degree reflections & 0 \\
\hline Number of grazing incidence reflections & 3 \\
\hline Wavelength matching factor & $98 \%$ \\
\hline Condenser throughput & $50 \%$ \\
\hline Total optical system throughput & $0.040 \%$ \\
\hline Overfill factor & 2 \\
\hline Illuminated area & $788 u^{\wedge} 2$ \\
\hline Illumination NA & 0.050 \\
\hline In-band EUV energy required into condenser per exposure & $94.41 \mathrm{uJ}$ \\
\hline
\end{tabular}

Table 3

Throughput budget for EUV/Visible light system to achieve 1\% noise level in the measured image.

Using the étendue arguments described in section 4 above we now proceed to calculate the minimum power requirements for two different source sizes. Recall that étendue calculations assume a perfect loss-free optical system, thus the calculations below are based on the assumption of ideal throughput. In reality the condenser throughput may be less than ideal due, for example, to ray losses in the condenser system (the contribution due to reflection from 2 condenser mirrors has already been taken into account). 


\begin{tabular}{|c|c|c|c|}
\hline In-band EUV energy required into condenser per exposure & 94.41 & & \\
\hline Sources & 200um & 20um & \\
\hline Source size (diameter) & 200 & 20 & um \\
\hline Source area & 31410 & 314.1 & $u m^{\wedge} 2$ \\
\hline Collectable NA (etendue limited) & 0.008 & 0.079 & \\
\hline Collection angle (etendue limited) & 0.45 & 4.54 & deg \\
\hline Fraction of $2 \mathrm{Pi}$ collected (etendue limited) & $0.0031 \%$ & $0.3140 \%$ & \\
\hline Minimum In-band EUV energy required into $2 \mathrm{Pi}$ per exposure & 3011.14 & 30.06 & mJ \\
\hline Exposure time & 1.00 & & sec \\
\hline Required in-band EUV power into 2Pi during exposure & $3.01^{*}$ & $0.03^{*}$ & w \\
\hline Conversion efficiency & $0.50 \%$ & & \\
\hline $\begin{array}{l}\text { Driver power during exposure } \\
{ }^{*} \text { For } 1 \% \text { noise in a } 1 \mathrm{sec} \text { exposure. }\end{array}$ & $602^{*}$ & $6^{*}$ & $\mathbf{W}$ \\
\hline
\end{tabular}

Table 4

Source power requirements to achieve $1 \%$ noise level in a $1 \mathrm{~s}$ exposure.

Such source powers, whilst somewhat on the high side, are not unreasonable. Note in particular the strong motivation to use a small source for the AIM tool due to étendue limitations placed on collectable power. This power level is driven by the specification of detector $1 \%$ noise over a $1 \mathrm{sec}$ exposure and can be relaxed if the desired noise level is also relaxed, as illustrated in the box below:

\begin{tabular}{|l|l|l|l|}
\hline $200 \mu \mathrm{m}$ diameter source & $1 \%$ noise & $2 \%$ noise & $3 \%$ noise \\
\hline $1 \mathrm{~s}$ exposure & $3 \mathrm{~W}$ & $0.75 \mathrm{~W}$ & $0.3 \mathrm{~W}$ \\
\hline $10 \mathrm{~s}$ exposure & $0.3 \mathrm{~W}$ & $0.075 \mathrm{~W}$ & $0.03 \mathrm{~W}$ \\
\hline
\end{tabular}

Table 5

In-band EUV power into $2 \pi$ per exposure for a $200 \mu \mathrm{m}$ diameter source to achieve a desired noise level in the final image (data taken from above spreadsheet). The 10 s exposure time is included for illustration-ideally the exposure time should be $<1$ sec for a practical system but is open to negotiation between vendor and client

It is therefore important to recognise that the power requirements listed above are part of a cost-benefit tradeoff between exposure time, noise levels, and source availability, and that source power requirements increase significantly as the target noise level is reduced. Furthermore, as noise levels can be reduced by increasing integration time, it may be desirable to design a two-mode system with a fast, higher noise scanning mode for navigation and a slower, low-noise mode for collecting AIM data. Rebinning the data so that four pixels are reduced to one pixel provides another avenue for decreasing noise and/or source power at the expense of spatial resolution and may be attractive for use in navigation mode. Such tradeoffs are a necessary part of the design process and will have to be optimised by each vendor individually.

\subsubsection{Technical risks and key comptetencies required of vendor}

Technical aspects of the scintillator, visible light microscope and CCD technologies are well understood and these components can be sourced from vendors and readily integrated into an AIM platform. The main areas of technical risk appear to be uncertainty about the conversion efficiency, which directly links to optical throughput and required source parameters, and some uncertainty about the degradation in spatial resolution caused by using a visiblelight microscope as the second stage.

In addition to the general system competencies required for system integration the vendor should demonstrate competency in the following areas relevant to this design approach:

- An optical design which emulates the salient features of a 6-mirror optical system (ie: matches the MTF)

- Ability to fabricate the required EUV optics to sufficient quality or source them from a vendor

- Demonstration of spatial resolution of the visible light chain, and analysis of how this will affect the fidelity of the aerial image measurement 
- Demonstration or verification of the total throughput of the visible light side of the optical system for verification of the throughput budget

- Demonstration of either practicability of using the scintillator as a vacuum window or the imaging effects of having the microscope objective in vacuum and projecting the visible light image through a vacuum viewport.

\subsubsection{Technology development or technology transfer requirements}

The required technologies appear to be commercially available from vendors at this point in time. However, for the purposes of AIM system integration we suggest that a vendor should demonstrate the capability of the scintillator/visible light system they intend to use as a first step. A key metric of performance would be to verify the $\mathrm{PSF} / \mathrm{LSF} / \mathrm{MTF}$ of the scintillator/microscope combination to be used - for example using a knife-edge or pinhole shadow cast onto the scintillator to demonstrate the imaging properties of the second magnification stage. This could then be combined with the MTF of the EUV optics to determine the overall performance of the optical system.

The efficiency of the particular scintillator/microscope combination to be used should also be verified so as to validate the conversion efficiency figures used in the throughput budget. Whilst it would be preferable to do this in the configuration to be used in the AIM system a well-designed experiment from which the actual conversion efficiency can be determined would suffice. This can be done as a preliminary benchtop experiment and could be performed in conjunction with the resolution test mentioned above.

The vendor of the EUV optical components must also demonstrate the capacity to manufacture EUV optics of the required optical quality. Both SVG Tinsley and Zeiss have demonstrated this capacity to the VNL, but this does not preclude sourcing the optics from another vendor.

\subsubsection{Useful references}

1. B.LaFontaine et.al. "Submicron soft X-ray fluorescence imaging" (1995) 66(3) Appl.Phys.Lett. 282

2. A.Koch et.al. "X-ray imaging with sub-micrometer resolution employing transparent luminescent screens" (1998) 15(7) J.Opt.Soc.Am.A. 1940

3. D.Husk et.al. "Quantum efficiency and linearity of 19 phosphors in the soft x-ray regime" (1992) 9(5) J.Opt.Soc.Am.B. 660

4. FAQ page on StarTech web site (http://www.startechinst.com/bifaw.htm) 


\subsection{Electron microscope (PEEM) based system}

Combining an EUV objective with a high resolution electron microscope for secondary magnification provides the capability to obtain high magnification using electron optics, relaxing the magnification requirements of the EUV objective. In this configuration, illustrated schematically in Figure 38 below, the EUV image from an AIM mode objective is projected onto a photocathode that converts the EUV image into a photoelectron image which is further magnified using an electron microscope column. The advantage of this design is that it may provide the most rapid integration path for using an existing low-magnification EUV optics, for example the 5x MET adapted for AIM use.

A system concept design for system integration using PEEM secondary magnification is illustrated below:

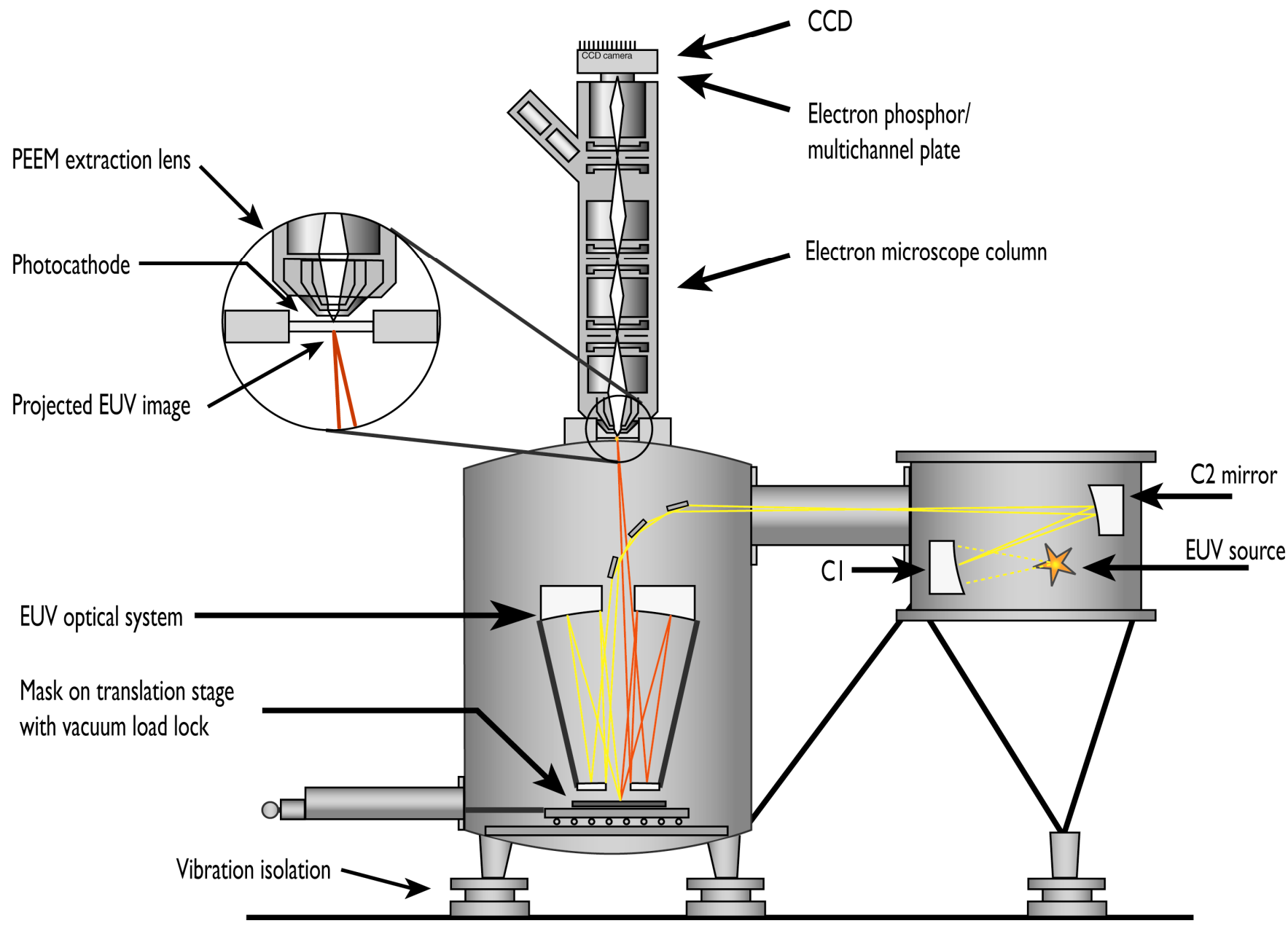

Figure 38

Conceptual layout of an EUV-PEEM based AIM tool

\subsubsection{Critical subsystem description}

Key components specific to this system are:

- Photo-electron emission microscope (PEEM) for secondary magnification

- Photocathode for converting the EUV image to electrons

- AIM-compatible EUV optics to image the mask onto the scintillator

\subsubsection{PEEM system}

PEEM systems are readily available from a number of commercial vendors, for example Staib (http://staib.com). For the purposes of this discussion we consider using a Staib PEEM column so that performance specifications and system tradeoffs for a specific system can be discussed. However this choice is made only for the 
purposes of this study and it is open to a Aim tool vendor to choose another supplier for the PEEM system provided the performance characteristics of that system are properly studied with respect to AIM mode implementation.

Data from the vendor indicates that the PEEM system is capable of high magnification of 300-1000x with demonstrated $200 \mathrm{~nm}$ spatial resolution over a nominal $150 \mu \mathrm{m}$ field of view. However there are practical tradeoffs with magnification and field of view that need to be considered as illustrated in Figure 39. As can be seen curvature of field is a significant effect over the full $400 \mu \mathrm{m}$ field of view. Whilst possibly useful for locating defects, it can be readily seen that field curvature limits the practical field of view for AIM applications to about $150 \mu \mathrm{m}$. According to the

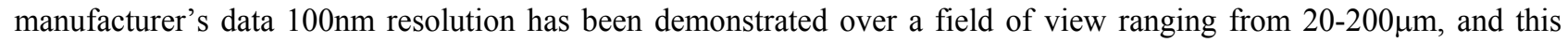

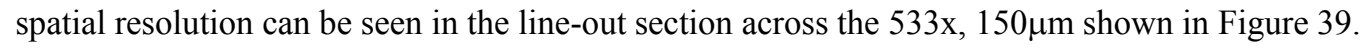

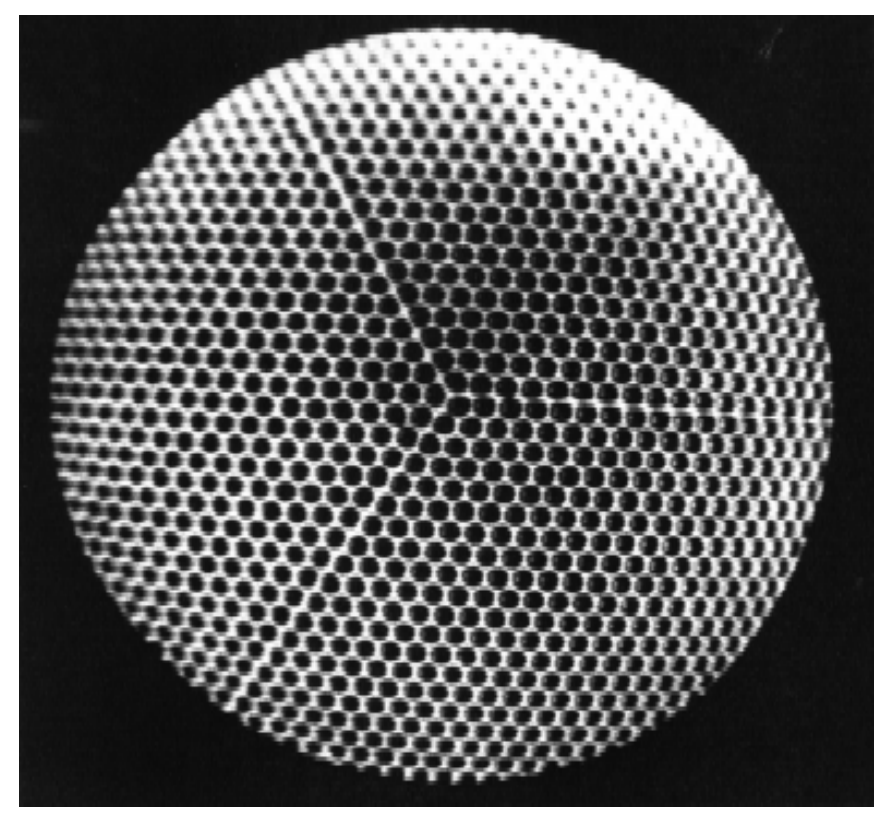

210x, 400 $\mathrm{m}$ field, significant curvature of field is evident

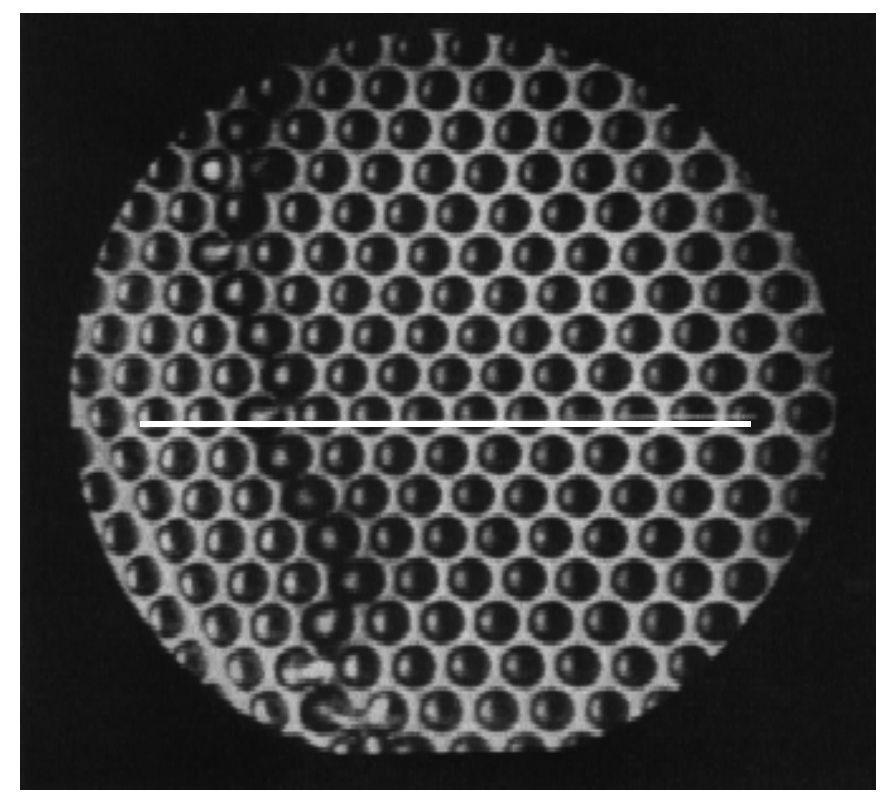

$533 x, 156 \mu \mathrm{m}$ field, field curvature more manageable

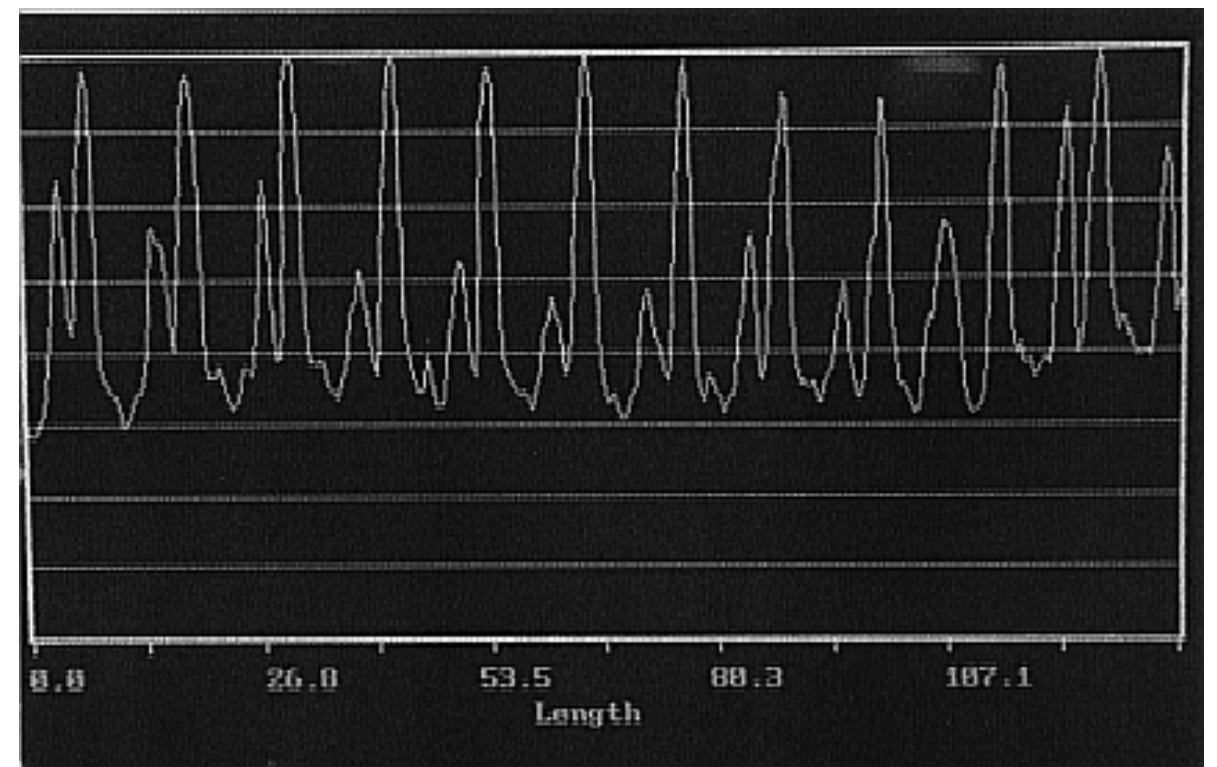

Line section through 533x image along line shown. Channel width is $10 \mu \mathrm{m}$ and the centre-to-centre spacing between channels is $12 \mu \mathrm{m}$.

\section{Figure 39}

Sample images of a microchannel plate illuminated with UV light taken with a Staib PEEM-350-10 system. The microchannel plate test object consists of a regular grid of $10 \mu \mathrm{m}$ channels arranged with a $12 \mu \mathrm{m}$ centre-to-centre distance, and the lineout section illustrates the spatial resolution of the system. Images and data extracted from Staib product literature. 
We note, however, that resolution, throughput and field of view depend heavily on electron column design. According to a Cerrina study (NIST paper) 10nm resolution at the photocathode is possible, but this comes at the expense of field of view and throughput of the optical column ${ }^{17}$.

\subsubsection{Microchannel plate and CCD}

Following magnification of the photoelectron image using a PEEM, most PEEM systems convert the electrons back to visible light photons before detection on a CCD chip. This is done using either an electron scintillator or a multichannel plate. The advantage of using a multichannel plate is that it provides gain, reducing the amount of time required make an exposure. This is useful when signal levels are low, however the effects of MCP plate sampling and gain have to be considered. With regard to gain, the MCP amplifies both the signal and the shot noise present in the incident electron image, thus increased MCP gain is accompanied by a noisier image. There is therefore a system noise trade-off to be made in using MCP plate gain to increase the intensity of the image - each exposure may take less time, but each image will be noisier requiring more images to be averaged in order to achieve a certain noise level. For AIM applications where a threshold resist model is applied to the measured data it is desirable to minimise the amount of noise in the image, motivating the use of very low MCP gain.

The issue of MCP sampling should also be considered. An MCP plate (see PEEM images in Figure 39) consist of discrete channels used to amplify the electron image. The MCP plate provides discrete sampling of the electron image before detection on the CCD, thus in an MCP system there are two stages of sampling during detection: at the MCP and CCD. This double-sampling inevitably has an effect on the system MTF which needs to be taken into account. Experiments verifying the sampling and signal-to-noise effects of the MCP on the PEEM image are therefore desirable as a part of system qualification. In this context we note that these measurements could be included in tests of the overall resolution, throughput and system noise of the photocathode/PEEM combination for system qualification as suggested below.

\subsubsection{Photocathode}

Photocathodes for converting the incident EUV light into electrons have been studied in the literature and their performance characterised ${ }^{18}$. For the application contemplated the photocathode must operate in transmission, and from studies in the literature the optimum electron yield for a transmission photocathode is obtained using 20-30nm thick CsI deposited on a $100 \mathrm{~nm}$ thick $\mathrm{Si}_{3} \mathrm{~N}_{4}$ membrane which is in turn held on an etched $\mathrm{Si}$ wafer for structural support. The photocathode itself can be manufactured using standard $\mathrm{Si}$ micro-machining techniques and produces a maximum electron yield for a $25 \mathrm{~nm}$ thick CsI film at 0.24 electrons per incident $13.5 \mathrm{~nm}$ EUV photon of between EUV objective. A cutaway schematic of the construction of such a photocathode, extracted from the literature ${ }^{19}$, is shown below in Figure 40 .

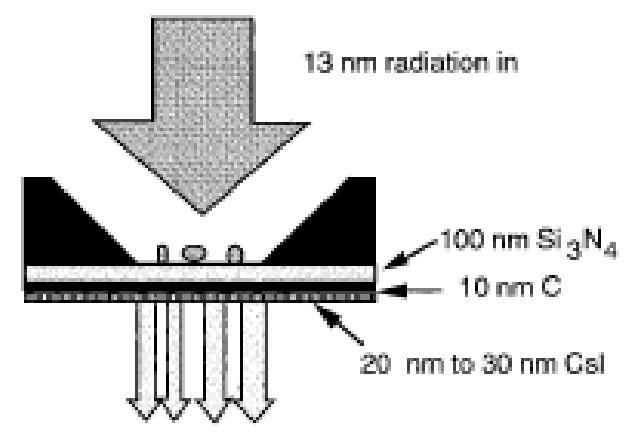

(a)

Secondary photoelectrons out

Figure 40

Schematic of the construction of a transmission EUV photocathode, taken from NIST paper.

\subsubsection{EUV objective design}

We turn now to the design of a suitable EUV objective for combination with a PEEM system. As mentioned above there is flexibility in the design and selection of magnification for the EUV optics stage, and it is open to the vendor to make choices on this subject to the requirement that the imaging is faithful to the imaging properties of a 6-

\footnotetext{
${ }^{17}$ Watts et.al. "A transmission x-ray microscope based on secondary electron imaging” (1997) 68 Rev.Sci.Instru. 3464.

${ }^{18}$ Ibid. See also S.Grantham, Masters’ Thesis, University of Central Florida, 1993.

${ }^{19}$ ibid.
} 
mirror stepper system. At first glance a Schwarzschild-type system appears attractive, however the strict Schwarzschild condition leads to an angle of incidence of $11.8^{\circ}$ at the mask, whilst Schwarzschild-type designs give an angle of incidence of $7.5^{\circ}$ at the mask. This leads to excessive bandwidth-shifting and shadowing in the image which is not meaningful for AIM mode imaging. Tilting the Schwarzschild potentially solves the angle of incidence problem, however this leads to the creation of excessive distortion and loss of contrast in the image. Furthermore tilting the mask with respect to the objective also tilts the image plane, and with the tilt of the image being the magnification times the tilt at the mask this rapidly leads to large tilts when operating at 10x magnification. The tilted object and image planes in turn lead to an significant change in magnification across the field, which is undesirable for an AIMS-type tool.

We are therefore led to explore MET-type designs for use in an AIM application. Because of the high magnification and spatial resolution possible with electron microscope optics is desirable to employ an EUV objective with relatively low magnification. This is particularly the case once the field of view of the PEEM system at the photocathode is taken into account. Recalling from above that the PEEM system discussed there was limited by field curvature to approximately a $150 \mu \mathrm{m}$ field of view at the photocathode, coupling this to a $5 \mathrm{x}$ objective would lead to a $30 \mu \mathrm{m}$ field of view at the mask - comparable to that anticipated for an AIM system. Increasing the objective magnification to $10 \mathrm{x}$ would halve this field of view at the mask to $15 \mu \mathrm{m}$. Whilst there may be other considerations involved in determining the magnification used, and the field of view of the PEEM used may differ from that of the one considered above, there is motivation to keep the magnification of the EUV objective stage as low as possible in order to maximise the field of view at the mask.

However, we refer to the discussion in section 3.4.5 on reusing the MET optics and note that the $5 \mathrm{x}$ magnification of the MET mean that it could be well suited to use as an AIM objective provided the compromises involved in changing the angle of incidence to $7^{\circ}$ and decreasing the wavelength from $13.5 \mathrm{~nm}$ to $13.47 \mathrm{~nm}$ were deemed acceptable. Alternatively, referring to the discussion in section 3.5 on novel AIM mode objectives, we note that it is possible to design an equal-radii system with moderate asphericity which supports AIM imaging $6^{\circ}$ angle of incidence to the mask with a $0.0625 \mathrm{NA}$ clear aperture and 5-10x magnification with a manageable track length of $600 \mathrm{~mm}$. The performance properties of this system were discussed in detail above so we do not repeat them here.

\subsubsection{Matching magnification through the system}

We now turn to the issue of matching magnification through the optical system. Vendors are free to choose the combination of magnification, sampling and field of view depending on the PEEM and objective design comtemplated for use in the AIM system. However one such example of how the PEEM and EUV magnification could be combined based on the above discussion is illustrated below.

In this example we note that the PEEM has $100 \mathrm{~nm}$ resolution at the photocathode plane, thus one would want to have at least $50 \mathrm{~nm}$ pixels in the photocathode plane to ensure adequate sampling of the image on the photocathode. The magnification of the PEEM is variable from 200-2000x depending on system design, thus this magnification is within the accessible range of the PEEM system. Assuming a 1024x1024 CCD camera and 50nm pixels gives a $50 \mu \mathrm{m}$ field of view at the photocathode, which with a $5 \mathrm{x}$ objective corresponds to a $10 \mu \mathrm{m}$ field of view on the mask. Working back up the system, the EUV objective will have $107 \mathrm{~nm}$ resolution at the mask which, at $5 \mathrm{x}$ magnification, magnifies each resolution element on the mask into $535 \mathrm{~nm}$ at the photocathode, sufficiently above the 100nm resolution of the PEEM system that spatial resolution of the PEEM should not have a significant effect on image quality. Noting that PEEM magnification has been adjusted so that each CCD pixel corresponds to $50 \mathrm{~nm}$ in the photocathode plane this gives an effective sampling of more than $8 \mathrm{CCD}$ pixels per AIM objective resolution element, which is well above the minimum Nyquist sampling requirement. 


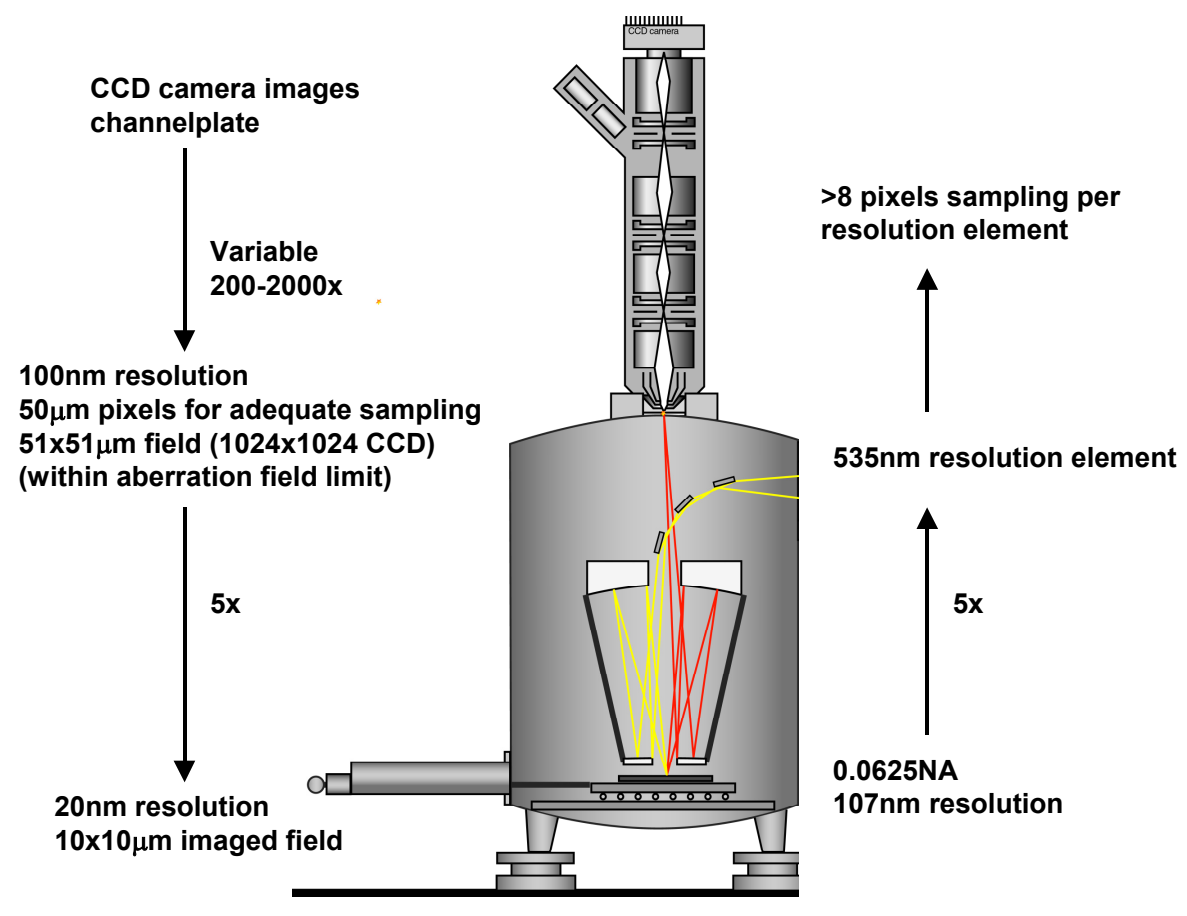

Figure 41

Example of matching spatial resolution in a PEEM system.

\subsubsection{System throughput budget}

We now proceed to calculate a preliminary throughput budget for a PEEM based AIM system in order to provide an comparative estimate of required source power for different configurations. Once again figures in this throughput budget represent the best estimate based on information to hand at the time of writing. Individual vendors may choose to use different values of certain parameters that will flow through the throughput budget once changed. For example a vendor may have a different estimate of anticipated multilayer mirror reflectivity, collection efficiency, CCD parameters or magnification, and once changed these figures will flow through the spreadsheet and affect the final numbers. In particular, we note that the value chosen for PEEM is a best estimate based on data available in the literature, but that this figure will depend strongly on PEEM design and the trade-off made by a vendor between resolution, throughput and field of view.

In order to determine the required source power, and to ensure consistency between different approaches, we aim for a noise level of $1 \%$ in the detected image. In this regard it is important to point out that the source powers here are indicative only and it may well be feasible to use a smaller source, although exposure times will have to be lengthened, a number of frames integrated, or the allowed noise level increased to compensate for the lower EUV flux. We also note that for this discussion we have chosen to use an electron phosphor instead of an MCP plate so as to minimise noise amplification and the effect of MCP sampling on the measured image. It is possible to greatly reduce throughput using gain on the MCP to compensate for other system losses, however we note that this comes at the expense of a higher noise level and additional sampling at the MCP plate. It is open to a vendor to choose to use an MCP instead of a phosphor to decrease the exposure time, however for the purposes of this study we choose to use a phosphor with a conservative conversion efficiency of 1.

Note also that in the interests of consistency we use the same values of common figures such as multilayer reflectivity as used in evaluating other systems so as to enable a fair side-by-side comparison of the various approaches. 


\begin{tabular}{|c|c|}
\hline \multicolumn{2}{|l|}{ Electron conversion system (PEEM) } \\
\hline \multicolumn{2}{|l|}{ Preliminary throughput budget } \\
\hline & Value \\
\hline \multicolumn{2}{|l|}{ Target values } \\
\hline Noise level & $1 \%$ \\
\hline Exposure time & $1 \mathrm{sec}$ \\
\hline \multicolumn{2}{|l|}{ Visible light CCD } \\
\hline Total system noise (total shot noise) & $1.06 \%$ \\
\hline CCD type & KAF-1400 \\
\hline Pixel size & $6.8 \mathrm{um}$ \\
\hline Electrons per full well & 40,000 \\
\hline Measured number of electrons & 20,000 \\
\hline Array size & $1317 \times 1035$ \\
\hline Number of pixels (total) & $1,363,095$ \\
\hline electrons produced per incident photon & 1.00 \\
\hline Quantum efficiency & $60 \%$ \\
\hline Visible photons per pixel required for a full well & 33,333 \\
\hline \multicolumn{2}{|l|}{ Multi-channel plate/electron phosphor } \\
\hline Gain (conversion efficiency, photons per incident electron) & 1.00 \\
\hline Electrons incident on MCP/phosphor to fill well & 33,333 \\
\hline Shot noise contribution & $0.55 \%$ \\
\hline \multicolumn{2}{|l|}{ Electron microscope column } \\
\hline Microscope magnification & $200 x$ \\
\hline Transmission efficiency & $90 \%$ \\
\hline Fraction of $4 \mathrm{Pi}$ electron emission collected & $50.0 \%$ \\
\hline Electron microscope efficiency & $45.00 \%$ \\
\hline \multicolumn{2}{|l|}{ Photocathode } \\
\hline Pixel size at photocathode & $34 \mathrm{~nm}$ \\
\hline Field of view of PEEM on photocathode (square) & $40 \mathrm{um}$ \\
\hline Electrons emitted per EUV photon (into 4Pi) & 0.2 \\
\hline Number of electrons emitted into 4Pi per pixel to fill well & 74,074 \\
\hline Number of incident EUV photons needed per pixel & 370,370 \\
\hline Incident EUV energy required per pixel & $3.70 \mathrm{E}+07 \mathrm{eV}$ \\
\hline EUV energy incident on scintillator (over field) & $5.05 \mathrm{E}+13 \mathrm{eV}$ \\
\hline EUV energy incident on scintillator (over field) & $8.08 \mathrm{uJ}$ \\
\hline \multicolumn{2}{|l|}{ Projection optics } \\
\hline Magnification & $5 x$ \\
\hline Collected NA & 0.0625 \\
\hline Nominal resolution & $107 \mathrm{~nm}$ \\
\hline Resolution unit size on scintillator & $536 \mathrm{~nm}$ \\
\hline Mirror reflectivity - normal incidence & $70 \% \%$ \\
\hline Number of normal incidence reflections & 2 \\
\hline Wavelength matching factor & $99 \%$ \\
\hline Projection optic throughput & $49 \%$ \\
\hline \multicolumn{2}{|l|}{ Mask } \\
\hline CCD pixel size at mask & $6.8 \mathrm{~nm}$ \\
\hline Pixels per resolution element (sampling) & 15.8 \\
\hline Field of view (square) & 7.94 um \\
\hline Reflectivity of mask & $65 \%$ \\
\hline Wavelength matching factor & $98 \%$ \\
\hline
\end{tabular}




\begin{tabular}{|c|c|}
\hline Overall mask throughput & $64 \%$ \\
\hline Energy incident on mask over field per exposure & $26.14 \mathrm{uJ}$ \\
\hline \multicolumn{2}{|l|}{ System loss factors } \\
\hline Spectral purity filter throughput & $35 \%$ \\
\hline Transmission through gas & $50 \%$ \\
\hline Transmission through debris shield & $80 \%$ \\
\hline Throughput contribution of system loss factors & $14 \%$ \\
\hline \multicolumn{2}{|l|}{ Condenser } \\
\hline Coherence (sigma) & 0.8 \\
\hline Mirror reflectivity - normal incidence & $70 \%$ \\
\hline Mirror reflectivity - 45 degree & $32 \%$ \\
\hline Mirror reflectivity - grazing incidence & $90 \%$ \\
\hline Number of normal incidence reflections & 1 \\
\hline Number of 45 degree reflections & 0 \\
\hline Number of grazing incidence reflections & 3 \\
\hline Wavelength matching factor & $98 \%$ \\
\hline Condenser throughput & $50 \%$ \\
\hline Total optical system throughput & $0.195 \%$ \\
\hline Overfill factor & 2 \\
\hline Illuminated area & $126 u^{\wedge}{ }^{\wedge}$ \\
\hline Illumination NA & 0.050 \\
\hline In-band EUV energy required into condenser per exposure & $746.73 \mathrm{uJ}$ \\
\hline
\end{tabular}

Table 6

Throughput budget for EUV/PEEM system to achieve 1\% noise level in the measured image.

Using the étendue arguments described in section 4 above we now proceed to calculate the minimum power requirements for two representative source sizes. Recall that étendue calculations assume a perfect loss-free optical system, thus the calculations below are based on the assumption of ideal throughput. In reality the condenser throughput may be less than ideal due, for example, to ray losses in the condenser system (the contribution due to reflection from 2 condenser mirrors has already been taken into account).

\begin{tabular}{|c|c|c|c|}
\hline In-band EUV energy required into condenser per exposure & 746.73 & & \\
\hline Sources & 200um & 20um & \\
\hline Source size (diameter) & 200 & 20 & um \\
\hline Source area & 31410 & 314.1 & $u m^{\wedge} 2$ \\
\hline Collectable NA (etendue limited) & 0.003 & 0.032 & \\
\hline Collection angle (etendue limited) & 0.18 & 1.82 & deg \\
\hline Fraction of $2 \mathrm{Pi}$ collected (etendue limited) & $0.0005 \%$ & $0.0502 \%$ & \\
\hline Minimum In-band EUV energy required into 2Pi per exposure & 148849 & 1488 & mJ \\
\hline Exposure time & 1.00 & & $\sec$ \\
\hline Required in-band EUV power into 2Pi during exposure & 148.85 & 1.49 & w \\
\hline Conversion efficiency & $0.50 \%$ & & \\
\hline Driver power during exposure & 29770 & 297.62 & $\mathbf{w}$ \\
\hline
\end{tabular}

Table 7

Source power requirements to achieve $1 \%$ noise level in a $1 \mathrm{~s}$ exposure. 
These source powers are significantly larger than required for the other systems considered in this design study indicating that it is necessary to increase the throughput of the system in order design a practical system. This can be done by careful selection of PEEM operating conditions to maximise throughput and by including an MCP plate to add gain into the system. The effect of MCP gain is linear with power - a factor of 100 MCP gain would reduce the required source power by a factor of 100, but also increase the level of noise in the image. The power levels described above are driven by the specification of detector $1 \%$ noise over a $1 \mathrm{sec}$ exposure and can be relaxed if the desired noise level is also relaxed, as illustrated in Table 8 below.

\begin{tabular}{|l|l|l|}
\hline $200 \mu \mathrm{m}$ diameter source & Source power & Noise level \\
\hline x1 MCP gain & $148 \mathrm{~W}$ & $1 \%$ \\
\hline x10 MCP gain & $14.8 \mathrm{~W}$ & $2 \%$ \\
\hline x100 MCP gain & $1.48 \mathrm{~W}$ & $5.7 \%$ \\
\hline x1000 MCP gain & $0.15 \mathrm{~W}$ & $18 \%$ \\
\hline
\end{tabular}

Table 8

In-band EUV power into $2 \pi$ per exposure for a $200 \mu \mathrm{m}$ diameter source as a function of MCP gain, and the corresponding level of noise in the detected image (data taken from above spreadsheet).

Inspection of Table 8 clearly shows how the addition of gain in the multi-channel plate (MCP) can significantly decrease the required source power, at the expense of higher noise levels in the final image. From the above calculations it appears possible to build a PEEM system for use with a compact source provided an MCP is used to amplify the image intensity, however it is also apparent that this comes at the expense of an increase in noise level in the measured image.

It is therefore important to recognise that, as with the visible light conversion system, the power requirements listed above are part of a cost-benefit tradeoff between exposure time, noise levels, and source availability, and that source power requirements increase significantly as the target noise level is reduced. Furthermore, as noise levels can be reduced by increasing integration time, it may be desirable to design a two-mode system with a fast, higher noise scanning mode for navigation and a slower, low-noise mode for collecting AIM data. Rebinning the data so that four pixels are reduced to one pixel provides another avenue for decreasing noise and/or source power at the expense of spatial resolution and may be attractive for use in navigation mode. Such tradeoffs are a necessary part of the design process and will have to be optimised by each vendor individually. However, it appears is likely that some form of image intensification would be necessary in order to make the system compatible with a compact source.

\subsubsection{Technical risks and key comptetencies required of vendor}

Technical aspects of both the photocathode and PEEM design appear to be well understood, and it appears that these components can be either sourced from vendors or manufactured as needed for integration into an AIM microscope. The main areas of technical risk appear to be uncertainty about the conversion efficiency of the photocathode/PEEM system, which directly links to throughput and required source parameters, and some uncertainty about image degradation involved in using a PEEM for second stage magnification.

In addition to the general system competencies required for system integration the vendor should demonstrate competency in the following areas relevant to this design approach:

- An optical design which emulates the salient features of a 6-mirror optical system (ie: matches the MTF)

- Ability to fabricate the required EUV optics to sufficient quality or source them from a vendor

- Demonstration of spatial resolution of the PEEM column to be used across the proposed field of view and at the magnification to be used, and analysis of how this will affect the fidelity of the aerial image measurement

- Demonstration or verification of the total throughput of the photocathode/PEEM system for verification of the throughput budget

\subsubsection{Technology development or technology transfer requirements}

The required technologies for a PEEM-based system appear to be commercially available at this point in time. However, as with a visible light conversion system, for the purposes of AIM system integration we suggest that a vendor should demonstrate the capabilities of the photocathode/PEEM combination as a first step is system qualification. We note that throughput, field of view and resolution of a PEEM system are commonly dependent parameters, thus the experiments should be performed using a PEEM of the same design and at the same magnification as to be used in an AIM tool. Key metrics of system performance would be to:

1) verify the PSF/LSF/MTF of the scintillator/microscope combination to be used - for example using a knife-edge or pinhole shadow cast onto the scintillator to demonstrate the imaging properties of the second 
magnification stage. This could then be combined with the MTF of the EUV optics to determine the overall performance of the optical system.

2) verify the conversion efficiency and throughput of the proposed photocathode/PEEM system to validate the conversion efficiency figures used in the throughput budget. Whilst it would be preferable to do this in the configuration to be used in the AIM system a well-designed experiment from which the actual conversion efficiency can be determined would suffice. This can be done as a preliminary benchtop experiment and could be performed in conjunction with the resolution test mentioned above.

3) Performance with regard to both resolution and throughput should be verified across the field of view so as to confirm that field curvature effects will be negligible across the field of view on the photocathode. Verifying this figure will help determine the useable field of view on the mask when the system is combined with an EUV objective.

4) The tradeoffs involved in using a multichannel plate to increase throughput should be studied with regard to both noise amplification by the MCP and the decrease in spatial resolution caused by sampling the image at both the MCP plate and the CCD.

An EUV objective is also required to project an image of the mask onto the photocathode, thus the vendor of the EUV optical components must demonstrate the capacity to manufacture EUV optics of the required optical quality. We note that both Tinsley and Zeiss have demonstrated this capacity to the VNL, but this does not preclude sourcing the optics from another vendor.

\subsubsection{Useful references}

1. R.N.Watts, S.Liang, Z.H.Levine, T.B.Lucatoro, F.Polack and M.R.Scheinfein "A transmission x-ray microscope based on secondary electron imaging" (1997) 68 Rev.Sci.Instru. 3464.

2. Cerrina et.al. "Method and apparatus for X-ray and EUV inspection of lithography masks and other objects" US Patent 6002740, filed 2 Oct 1997, granted 14 Dec 1999.

3. S.Grantham, Masters' Thesis, University of Central Florida, 1993.

4. G.DeStasio, et.al. "An electron imaging approach to soft X-ray transmission spectromicroscopy" (1996) 67 Rev.Sci.Instrum 737.

5. F.Cerrina et.al. "Method and apparatus for X-ray and EUV inspection of lithography masks and other objects" US Patent 6002740, filed 2 Oct 1997, granted 14 Dec 1999.

6. T.Haga et.al. "At-wavelength EUV mask inspection using a Mirau interferometric microscope" (2000) 18 J.Vac.Sci.Technol.B. 2916.

7. M.Masaki et.al. "Planned X-ray imaging of the electron beam at the Spring-8 diagnostics beamline BL38B2" DIPAC 2001 proceedings, ESRF, Grenoble. 


\subsection{All-EUV system}

The previous two systems designs have involved converting the EUV radiation to some other form prior to secondary magnification. It is, however, possible to design a high-magnification system consisting of all normal incidence EUV mirrors for the purposes of an AIM design. In this section we seek to demonstrate the feasibility of designing such a system.

A conceptual layout of how an all-EUV system consisting of normal-incidence imaging optics may be designed is illustrated in Figure 42 below:

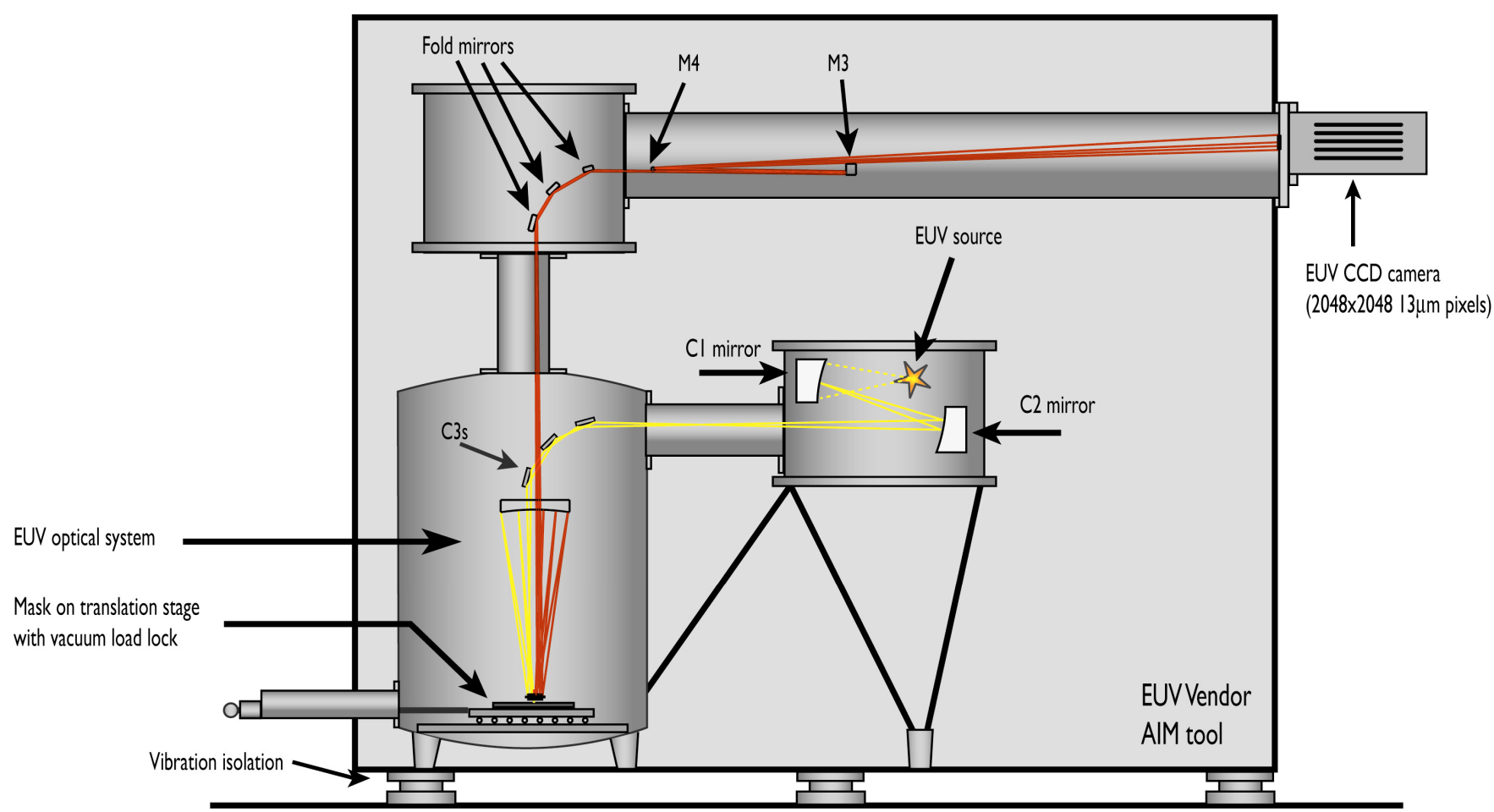

Figure 42

Conceptual layout of an all-EUV system.

\subsubsection{Critical subsystem description}

Key components specific to this technical approach are:

- A photometric quality EUV CCD camera

- EUV optics to image the mask onto the EUV CCD

\subsubsection{EUV CCD camera}

Soft X-ray CCD cameras sensitive to $13.5 \mathrm{~nm}$ are readily available from several vendors and pose minimum technical risk in this design. For this application we choose to use a thinned, back-illuminated CCD. To image a large area X-ray CCDs are available with phosphor screens and fibre-optic coupling to the CCD, however for direct imaging and to maximise spatial resolution it is preferable to use a CCD in which the EUV light is incident directly on the CCD chip.

For the purposes of this study we single out a PI-SX-1300 CCD camera available from Roper Scientific. This camera is a photometric-grade camera head available with a selection of CCD chips depending on application. The standard configuration has an EEV 36/40 CCD chip with a 1340x1024 array of $20 \mu \mathrm{m}$ pixels. For higher spatial resolution it is possible to use an EEV $42 / 40 \mathrm{CCD}$ with has a $2048 \times 2048$ array of $13.5 \mu \mathrm{m}$ pixels. Note that smaller pixel sizes provide higher resolution in the image plane and that front-illuminated visible light CCDs are available with pixel sizes down to $6.8 \mu \mathrm{m}$, however such small pixels are not commercially available for use in soft X-ray cameras. It may be possible to modify one of these chips for EUV use, but for the purposes of this study we choose to use the commercially available $13.5 \mu \mathrm{m}$ pixel EEV $42 / 40 \mathrm{CCD}$ chip as this provides highest spatial resolution in the detector 
plane and can be obtained directly from a vendor without modification. We note, however, that there are other manufacturers of CCD chips and camera heads and it is open to vendors to choose another CCD system as a part of system design.

\subsubsection{EUV optics}

A high-magnification imaging system is critical element in this design approach. Starting with the AIM-mode EUV objective design discussed in section 3.5 above and adding a second stage of magnification it is possible to design an EUV imaging system with sufficient magnification for imaging directly onto an EUV CCD with diffraction-limited performance across the field of view. The system design is sketched in Figure 43 below: note that the total track length required to obtain this magnification is $2.1 \mathrm{~m}$, thus three plane fold mirrors have been included in order to bend the system at right angles so it can fit in an enclosure of practical size. Note, however, that these fold mirrors could just as easily be replaced with a normal-incidence mirror to fold the system back on itself rather than projecting the image at right angles. Note that the composite RMS wavefront error of this system as designed is $1.8 \mathrm{~m} \lambda$ thus one would expect excellent contrast transfer from the system.

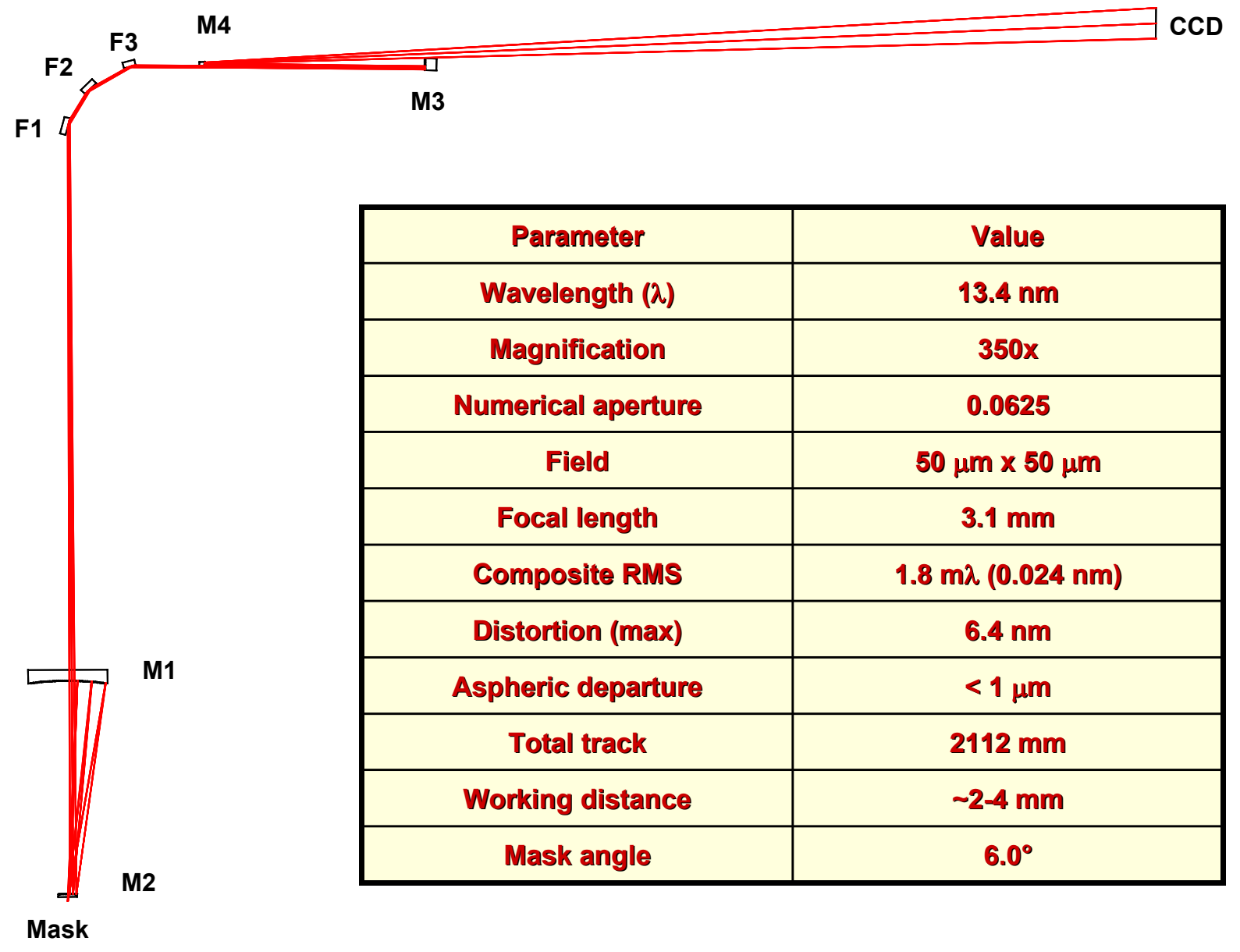

Figure 43

High-magnification EUV imaging system for AIM mode. The three plane fold mirrors have been included in order to bend the system at right angles so it can fit in an enclosure of practical size, as described in the text.

This system is based on two equal-radii systems placed back to back in order to stack the magnification, and the optical performance of this system is described in Figure 44 below. Note that the MTF response is uniform across the field of view and demonstrates diffraction-limited performance across the field of view. The through-focus response at $6250 \mathrm{lp} / \mathrm{mm}$ is symmetric and uniform across the field, thus one can expect this system to faithfully emulate the diffraction-limited performance of a stepper system through focus regardless of where the object is placed in the field of view. Inspection of the aerial image simulation in Figure 44 also reveals excellent diffraction-limited imaging performance across the field of view: the same conclusions regarding printability would be obtained regardless of 
where the object is placed in the field of view and this would be the same as anticipated for a stepper system of the same NA. Note in particular the different image slope for the outer bars - this is a diffraction-limited property of stepper systems representing iso-dense bias in the imaging system and has been faithfully captured by this AIM design.

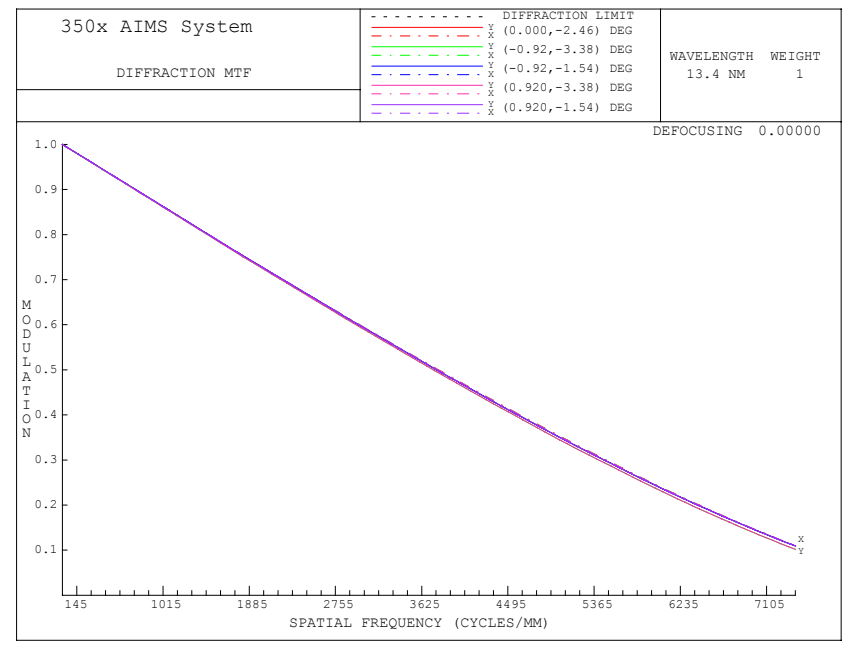

MTF response displays diffraction-limited performance across the field of view

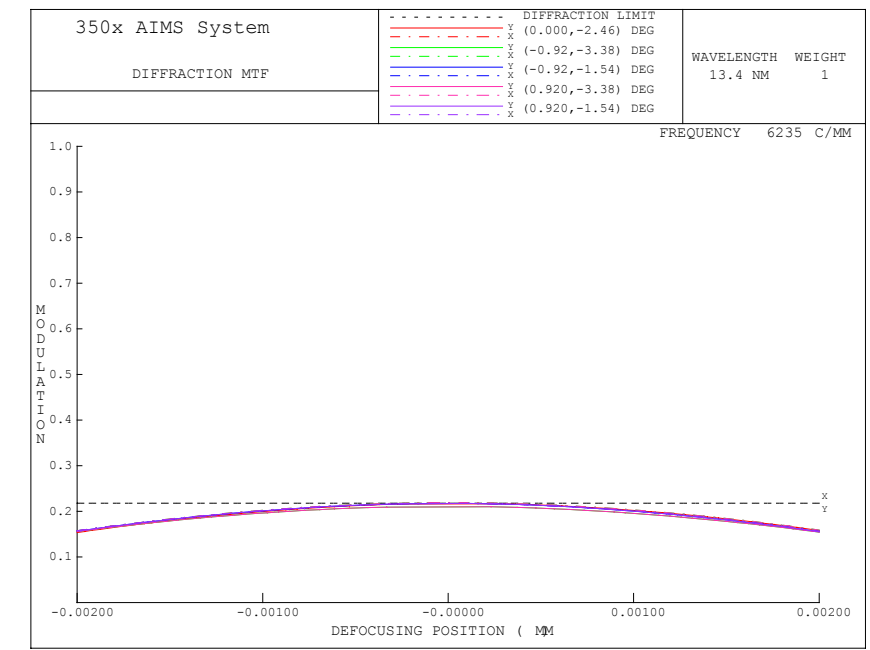

Through-focus contrast at $6250 \mathrm{lp} / \mathrm{mm}$ shows consistent performance across the field of view

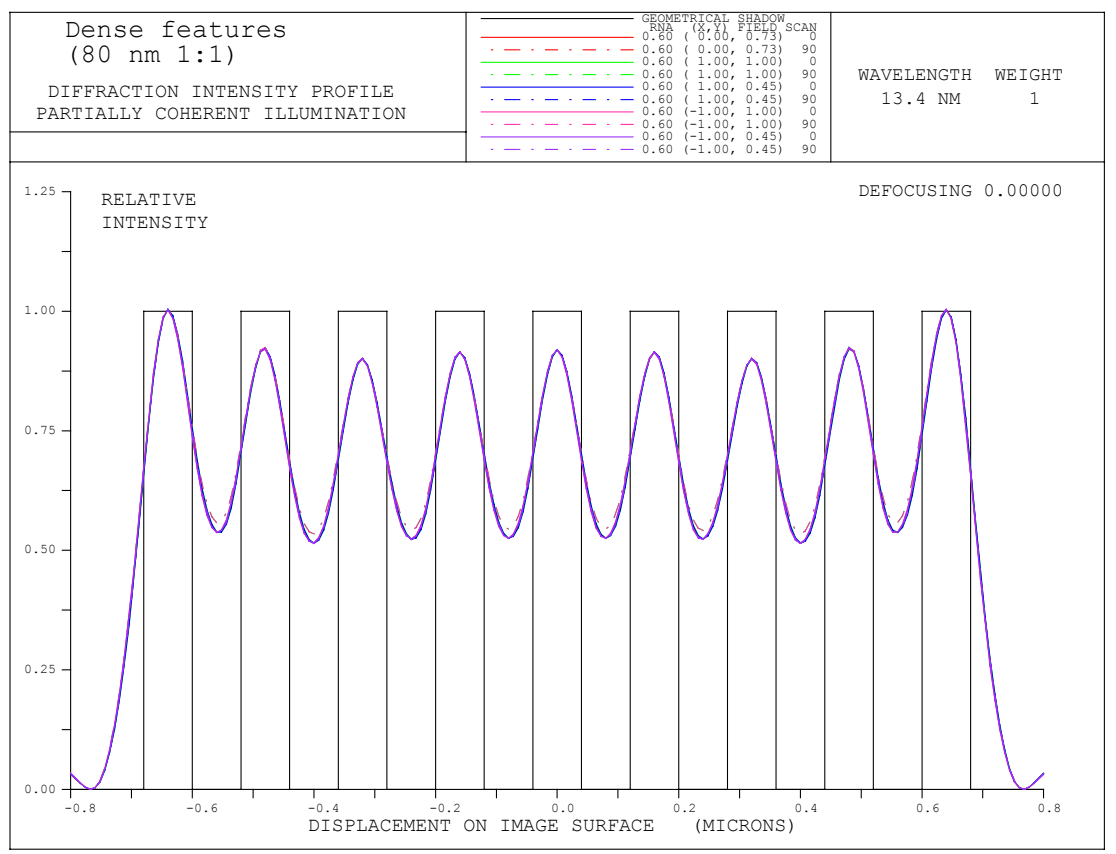

Partially coherent aerial image calculation at $6250 \mathrm{lp} / \mathrm{mm}$ demonstrates consistent diffraction-limited performance across the entire field of view. Conclusions about printability would be identical to that in a $0.25 \mathrm{NA}$ stepper system regardless of where the object is placed in the field of view.

Figure 44

Performance characteristics of 350x all-EUV optical design demonstrating consistent diffraction-limited performance across the entire field of view, delivering performance that accurately emulates the performance of a stepper system regardless of where the object is placed in the field of view.

The working distance for this system is $2-4 \mathrm{~mm}(8 \mathrm{~mm}$ from the reflecting surface of M2 minus $4-6 \mathrm{~mm}$ substrate thickness) allowing ample clearance from the mask. Mirror diameters of the parent optics are $84 \mathrm{~mm}$ for M1, $18 \mathrm{~mm}$ for M2, M3:24mm and M4:10mm, which is about the minimum size practical for polishing EUV aspheres based on experience with current EUV polishing technology. Note also that this excellent imaging performance is obtained with extremely mild aspheres on all optics of $<1 \mu \mathrm{m}$ aspheric departure across the clear aperture which is anticipated to simplify metrology of the optics. 
The geometric distortion for this system over the $50 \times 50 \mu \mathrm{m}$ field of view as designed is shown in Figure 45 below. Note that the distortion is bilaterally symmetric due to symmetries incorporated in the optical design, and that the distortion is less than $6.4 \mathrm{~nm}$ over the whole field. At 350x and using a CCD with $13.5 \mu \mathrm{m}$ pixels this distortion corresponds to less than 1/5th of a pixel maximum, sufficiently small for CD measurements to be made with confidence at any point in the visible field. We note, however, that it is likely that further reduction in the distortion could be made through further refinement of the design, although we choose not to pursue this for the purposes of this design study.

\section{Image Distortion Vs. Object Height}

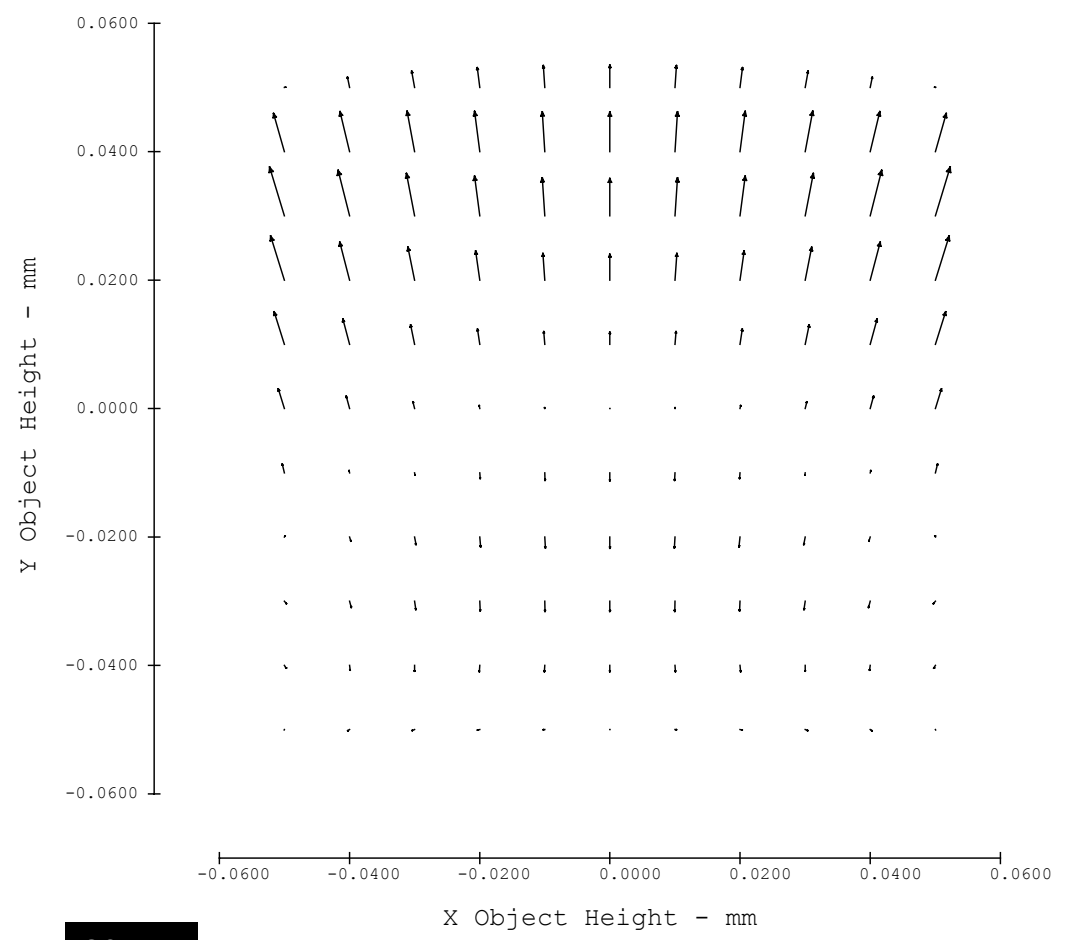

$20 \mathrm{~nm}$

Figure 45

Map of geometric distortion across the $50 \times 50 \mu \mathrm{m}$ field of view. Maximum distortion is $6.4 \mathrm{~nm}$ and is bilaterally symmetric due to design symmetries. Capacity for further reduction in distortion through design refinement is anticipated.

\subsubsection{Preliminary throughput budget}

We now proceed to calculate a preliminary throughput budget for an all EUV system using normal incidence optics. Note that the figures in this throughput budget represent the best estimate based on information to hand at the time of writing. Individual vendors may choose to use different values of certain parameters that will flow through the throughput budget once changed. For example a vendor may have a different estimate of anticipated multilayer mirror reflectivity, collection efficiency, CCD parameters or magnification, and once changed these figures will flow through the spreadsheet and affect the final numbers. This is to be expected and vendors are free to use different values appropriate to their particular design and manufacturing processes.

In order to determine the required source power, and to ensure consistency between different approaches, we aim for a noise level of $1 \%$ in the detected image. In this regard it is important to point out that the source powers here are indicative only and it may well be feasible to use a smaller source, although exposure times will have to be lengthened, a number of frames integrated, or the allowed noise level increased to compensate for the lower EUV flux. In the interests of consistency we use the same values of common figures such as multilayer reflectivity as used in evaluating other systems so as to enable a fair side-by-side comparison of the various approaches, and we calculate the throughput budget for a CCD camera with 2048x2048, 13.5 micron pixels as contemplated above, although it is open to a vendor to select another CCD for AIM applications. 


\begin{tabular}{|c|c|}
\hline \multirow{2}{*}{\multicolumn{2}{|c|}{$\begin{array}{l}\text { All EUV system } \\
\text { Preliminary throughput budget }\end{array}$}} \\
\hline & \\
\hline & $\underline{\text { Value }}$ \\
\hline \multicolumn{2}{|l|}{ Target values } \\
\hline Noise level & $1 \%$ \\
\hline Exposure time & $1 \mathrm{sec}$ \\
\hline EUV CCD & EEV 42/40 \\
\hline Total system noise (total shot noise) & $1.15 \%$ \\
\hline Pixel size & $13.5 \mathrm{um}$ \\
\hline Electrons per full well & 100,000 \\
\hline Measured number of electrons & 100,000 \\
\hline eV/photon & 3.65 \\
\hline electrons/photon & 27.40 \\
\hline photons per full well & 3650 \\
\hline Quantum efficiency (QE) & $0.45 \%$ \\
\hline Photons incident on a pixel & 8111 \\
\hline Photon energy incident on a pixel & $811,111 \mathrm{eV}$ \\
\hline Number of pixels (array) & $2048 \times 2048$ \\
\hline Number of pixels (total) & $4,194,304$ \\
\hline Energy incident on CCD & $3.40 \mathrm{E}+12 \mathrm{eV}$ \\
\hline EUV energy incident on whole CCD for full exposure & $0.54 \mathrm{uJ}$ \\
\hline \multicolumn{2}{|l|}{ Projection optics } \\
\hline Magnification & $350 x$ \\
\hline Collected NA & 0.0625 \\
\hline Nominal resolution & $107 \mathrm{~nm}$ \\
\hline Mirror reflectivity - normal incidence & $70 \%$ \\
\hline Mirror reflectivity - 45 degree & $32 \%$ \\
\hline Mirror reflectivity - grazing incidence & $90 \%$ \\
\hline Number of normal incidence reflections & 4 \\
\hline Number of 45 degree reflections & 0 \\
\hline Number of grazing incidence reflections & 0 \\
\hline Wavelength matching factor & $98 \%$ \\
\hline Projection optic throughput & $24 \%$ \\
\hline \multicolumn{2}{|l|}{ Mask } \\
\hline CCD pixel size at mask & $38.6 \mathrm{~nm}$ \\
\hline Pixels per resolution element (sampling) & 2.8 \\
\hline Field of view (square) & 79.0 um \\
\hline Reflectivity of mask & $65 \%$ \\
\hline Wavelength matching factor & $98 \%$ \\
\hline Mask transmission factor & $64 \%$ \\
\hline Energy incident on mask over field per exposure & $3.63 \mathrm{uJ}$ \\
\hline \multicolumn{2}{|l|}{ System loss factors } \\
\hline Spectral purity filter throughput & $35 \%$ \\
\hline Transmission through gas & $50 \%$ \\
\hline Transmission through debris shield & $80 \%$ \\
\hline Throughput contribution of system loss factors & $14 \%$ \\
\hline \multicolumn{2}{|l|}{ Condenser } \\
\hline Coherence (sigma) & 0.8 \\
\hline Illuminating NA & 0.05 \\
\hline Mirror reflectivity - normal incidence & $70 \%$ \\
\hline
\end{tabular}




\begin{tabular}{|lc|}
\hline Mirror reflectivity - 45 degree & $32 \%$ \\
Mirror reflectivity - grazing incidence & $90 \%$ \\
Number of normal incidence reflections & 1 \\
Number of 45 degree reflections & 0 \\
Number of grazing incidence reflections & 3 \\
Wavelength matching factor & $98 \%$ \\
Condenser throughput & $\mathbf{5 1 \%}$ \\
& $\mathbf{1 . 0 7 \%}$ \\
Total optical system throughput & 2 \\
Overfill factor & $12480 \mathrm{um}^{\wedge} 2$ \\
Illuminated area & 0.05 \\
Illumination NA & $\mathbf{1 0 1 . 6 7} \mathbf{~ u J ~}$ \\
In-band EUV energy required into condenser per exposure & \\
\hline
\end{tabular}

Table 9

Throughput budget for an all EUV reflective optics system to achieve 1\% noise level in the measured image.

Using the étendue arguments described in section 4 above we now proceed to calculate the minimum power requirements for two representative source sizes. Recall that étendue calculations assume a perfect loss-free optical system, thus the calculations below are based on the assumption of ideal throughput. In reality the condenser throughput may be less than ideal due, for example, to ray losses in the condenser system (the contribution due to reflection from 2 condenser mirrors has already been taken into account).

\begin{tabular}{|c|c|c|}
\hline \multirow{2}{*}{$\begin{array}{l}\text { In-band EUV energy required into condenser per exposure } \\
\text { Sources }\end{array}$} & \multicolumn{2}{|c|}{$101.67 \mathrm{uJ}$} \\
\hline & & \\
\hline Nominal source size (diameter) & 200 & $20 \mathrm{um}$ \\
\hline Source area & 31410 & $314 u^{\wedge} 2$ \\
\hline Collectable NA (etendue limited) & 0.032 & 0.315 \\
\hline Collection angle (etendue limited) & 1.81 & $18.37 \mathrm{deg}$ \\
\hline Fraction of $2 \mathrm{Pi}$ collected (etendue limited) & $0.0497 \%$ & $5.1 \%$ \\
\hline Minimum In-band EUV energy required into $2 \mathrm{Pi}$ per exposure & 205 & $1.99 \mathrm{~mJ}$ \\
\hline Exposure time & 1.00 & $\mathrm{sec}$ \\
\hline Required in-band EUV power into $2 \mathrm{Pi}$ during exposure & $0.2^{*}$ & $0.0020^{*} \mathrm{~W}$ \\
\hline Conversion efficiency & $0.50 \%$ & \\
\hline $\begin{array}{l}\text { Driver power during exposure } \\
{ }^{*} \text { For } 1 \% \text { noise in a } 1 \mathrm{sec} \text { exposure. }\end{array}$ & $41^{*}$ & $0.40^{*} \mathrm{~W}$ \\
\hline
\end{tabular}

Table 10

Source power requirements for an all EUV reflective optics system to achieve $1 \%$ noise level in a 1 s exposure.

Such source powers are not unreasonable for a compact EUV source, although we once again note the strong motivation to use a small source for the AIM tool due to étendue limitations placed on collectable power. Note also that this system provides the lowest source requirements of all of the systems analysed in this study. This power level is driven by the specification of detector $1 \%$ noise over a $1 \mathrm{sec}$ exposure and can be relaxed if the desired noise level is also relaxed, as illustrated in Table 11 below: 


\begin{tabular}{|l|l|l|l|}
\hline $200 \mu \mathrm{m}$ diameter source & $1 \%$ noise & $2 \%$ noise & $3 \%$ noise \\
\hline $1 \mathrm{~s}$ exposure & $0.2 \mathrm{~W}$ & $0.07 \mathrm{~W}$ & $0.031 \mathrm{~W}$ \\
\hline $10 \mathrm{~s}$ exposure & $20 \mathrm{~mW}$ & $7 \mathrm{~mW}$ & $3.1 \mathrm{~mW}$ \\
\hline
\end{tabular}

Table 11

In-band EUV power into $2 \pi$ per exposure for a $200 \mu \mathrm{m}$ diameter source to achieve a desired noise level in the final image (data taken from above spreadsheet). The 10 s exposure time is included for illustration-ideally the exposure time should be $<1$ sec for a practical system and must be negotiated between vendor and client.

It is therefore important to recognise that the power requirements listed above are part of a cost-benefit tradeoff between exposure time, noise levels, and source availability, and that source power requirements increase significantly as the target noise level is reduced. Furthermore, as noise levels can be reduced by increasing integration time, it may be desirable to design a two-mode system with a fast, higher noise scanning mode for navigation and a slower, low-noise mode for collecting AIM data. Rebinning the data so that four pixels are reduced to one pixel provides another avenue for decreasing noise and/or source power at the expense of spatial resolution and may be attractive for use in navigation mode. Such tradeoffs are a necessary part of the design process and will have to be optimised by each vendor individually. 


\subsection{Potential zone plate implementations}

The discussion to this point has focussed on systems using normal-incidence multilayer coated optics, however it is also possible to use diffractive lenses, or zone plates, for EUV imaging. There may be a significant cost advantage in following this approach as a set of zone plates may cost on the order of $\$ 5,000$ to fabricate using established E-beam lithography techniques, significantly less than the anticipated cost of fabricating and coating normal-incidence multilayer optics. Although we do not choose to pursue a zone plate design for the purposes of this study, there are a number of issues particular to diffractive optics which must be addressed in order to evaluate the feasibility of this approach. The purpose of this section is to raise some of those issues in the same context as the preliminary system designs discussed to this point.

\subsubsection{On-axis and off-axis imaging systems}

The simplest zone plate design contemplates a single imaging element (zone plate) imaging the mask with a $0.0625 \mathrm{NA}$ zone plate tilted with respect to the mask so that the optical axis of the imaging system is at $6^{\circ}$ to the mask. The imaging system is therefore a tilted optical system and as such will suffer from keystone distortion and require a tilted image plane $e^{20}$. It is possible to correct for this by using an off-axis portion of an on-axis zone plate to eliminate keystone distortion and to have the object and image planes parallel to one another and the zone plate, as shown in Figure 46 below:

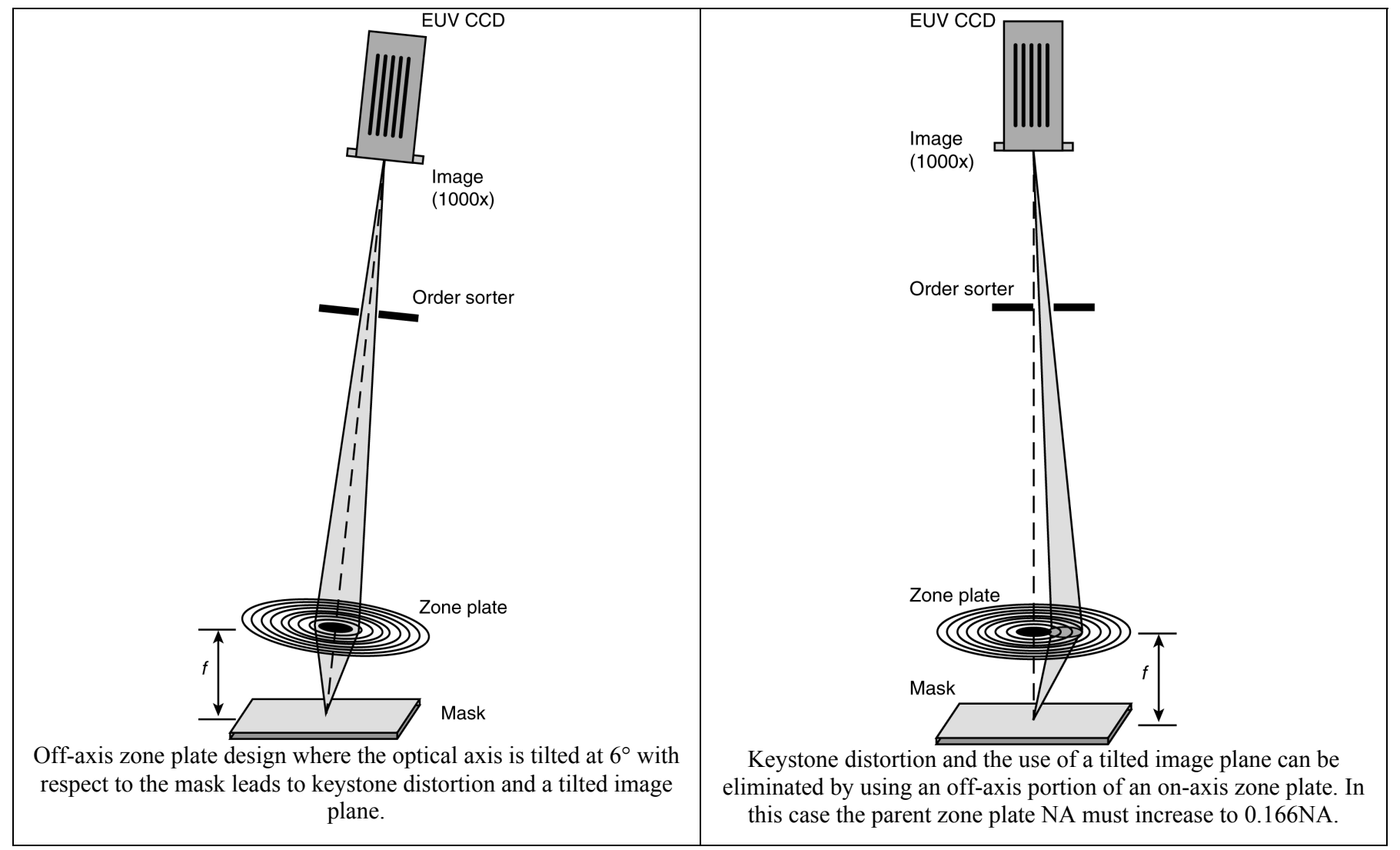

Figure 46

Off-axis and on-axis zone plate designs.

This on-axis zone plate design has the advantage that the zero and first order diffraction orders are physically separated, reducing flare in the system and allowing for more relaxed placement of the order sorting aperture. In order to capture the marginal ray of the $0.0625 \mathrm{NA}$ AIM pupil at $6^{\circ}$ angle of incidence using the on-axis design illustrated in Figure 46 it is necessary to have a parent zone plate with a numerical aperture of $0.166 \mathrm{NA}$. This is the design we choose to analyse in detail below.

\footnotetext{
${ }^{20}$ The effect of tilting an objective on keystone distortion, image plane tilt and through-focus behaviour was discussed in the section on tilted Schwarzschild concepts, and much of this discussion is also relevant to a tilted zone plate system.
} 


\subsubsection{Relationship between bandwidth, focal length and working distance}

As described in the down-selection report a zone plate is completely specified by the wavelength, bandwidth and resolution. In particular we have the following relationships:

$$
\begin{array}{ll}
\text { Outer zone width: } & \delta=\Delta r_{N}=\lambda / 2 N A \\
\text { Number of zones: } & N<\lambda / \Delta \lambda \\
\text { Focal length: } & f=4 N \Delta r_{N}^{2} / \lambda \\
\text { Diameter: } & D=4 N \Delta r_{N} \\
\text { Field of view: } & F O V \approx D / 3
\end{array}
$$

We therefore see that, unlike multilayer coated optics, the desired bandwidth, which sets the number of allowed zones $N$, plays a major role in determining the design of a zone plate system. For an AIM system we require a 0.0625NA

\begin{tabular}{|c|c|}
\hline \multicolumn{2}{|c|}{$2 \%$ bandwidth $=6$ mirror camera + mask and condenser optics } \\
\hline Parent NA: & $N A=0.166$ \\
\hline Outer zone width: & $\Delta r_{n}=40 \mathrm{~nm}$ \\
\hline Number of zones: & $N=50$ \\
\hline Focal length & $f=24 \mu m$ \\
\hline Diameter: & $D=8 \mu m$ \\
\hline Useable field: & $F O V=2.6 \mu \mathrm{m}$ \\
\hline \multicolumn{2}{|c|}{ Working distance from zone plate to mask: $24 \mu \mathrm{m}$} \\
\hline \multicolumn{2}{|c|}{$0.1 \%$ bandwidth $=$ LBL subfield exposure station } \\
\hline Parent NA: & $N A=0.166$ \\
\hline Outer zone width: & $\Delta r_{n}=40 \mathrm{~nm}$ \\
\hline Number of zones: & $N=1000$ \\
\hline Focal length & $f=477 \mu m$ \\
\hline Diameter: & $D=160 \mu m$ \\
\hline Useable field: & $F O V=53 \mu m$ \\
\hline Working distance $\mathrm{fl}$ & rom zone plate to mask: $0.47 \mathrm{~mm}$ \\
\hline
\end{tabular}
pupil giving a zone spacing $\Delta r_{n}=107 \mathrm{~nm}$ for all system. The wavelength is fixed at $13.5 \mathrm{~nm}$, thus the only undetermined parameter is the bandwidth. For a 6-mirror camera plus mask and condenser optics the bandwidth is anticipated to be $2 \%$, however for practical reasons a vendor may choose to operate at a different bandwidth.

For the on-axis design, the width of the outermost zone is $40 \mathrm{~nm}$ and we have:

Table 12

Design parameters for an on-axis zone plate design, parent $\mathrm{NA}=0.166$.

Inspection of the above table shows that, for an on-axis design, capturing the full $2 \%$ bandwidth of a stepper system leads to a very small working distance of $24 \mu \mathrm{m}$ between the mask and zone plate not including any additional clearance required for mounting hardware. This places the zone plate very close to the mask and requires demonstrating an accurate and reliable positioning system to avoid accidental contact with the mask. A more practical working distance of $0.5 \mathrm{~mm}$, which allows greater margin for error and is within the target range of $0.5-1 \mathrm{~mm}$ working distance, can be achieved by restricting the bandwidth to of the order of $0.1 \%$, as shown in the above table. This entails a significant departure from the bandwith of a production stepper system, thus the effect of reducing the bandwith on the equivalence between the measured AIM image and the result obtained in a stepper need to be carefully evaluated to ensure that no artefacts are inadvertently introduced through using narrower bandwidth illumination. The effect of bandwidth restriction on source requirements and condenser design must also be considered as it is necessary to design a condenser capable of providing the reduced bandwidth which, depending on source spectral properties, may in turn require discarding EUV photons from the source, thereby increasing the overall drive power required. 
For the sake of completeness we also provide calculations for the off-axis design by way of comparison:

\begin{tabular}{|ll|}
\hline $\mathbf{2 \%}$ bandwidth $=\mathbf{6}$ mirror camera + mask and condenser optics \\
Parent NA: & $N A=0.0625$ \\
Number of zones: & $N=50$ \\
Focal length & $f=170 \mu \mathrm{m}$ \\
Diameter: & $D=21 \mu \mathrm{m}$ \\
Useable field: & $F O V=7 \mu \mathrm{m}$ \\
Working distance from zone plate to mask: $170 \mu \mathrm{m}$ \\
Track length: $\quad 0.17 \mathrm{~m}$ for $1000 \mathrm{x}$ magnification \\
\hline Parent NA: & $N A=0.0625$ \\
Number of zones: & $N=1000$ \\
Focal length & $f=3.4 \mathrm{~mm}$ \\
Diameter: & $D=0.43 \mathrm{~mm}$ \\
Useable field: & $F O V=140 \mu \mathrm{m}$ \\
Working distance from zone plate to mask: $3.4 \mathrm{~mm}$ \\
Track length: $\quad 3.4 \mathrm{~m}$ for $1000 \mathrm{x}$ magnification \\
Parent NA: & $N A=0.0625$ \\
Number of zones: & $N=200$ \\
Focal length & $f=0.7 \mathrm{~mm}$ \\
Diameter: & $D=75 \mu \mathrm{m}$ \\
Useable field: & $F O V=25 \mu m$ \\
Working distance from zone plate to mask: $0.7 \mathrm{~mm}$ \\
Track length: & $0.7 \mathrm{~m}$ for $1000 \mathrm{x}$ magnification \\
\hline
\end{tabular}

Table 13

Design parameters for an off-axis zone plate design. Due to the reduced parent NA the focal lengths and bandwidth requirements of this system are different to that required for an on-axis design.

From this table it is apparent that a system which captures the full $2 \%$ bandwidth has a very small focal length $(170 \mu \mathrm{m})$ which means that the working distance from the zone plate to the mask would be of the order of $170 \mu \mathrm{m}$ not counting any mounting hardware. The only practical way to increase the focal length and, hence, the working distance to the mask is to decrease the bandwith as illustrated above. If this is done it is necessary to show through aerial image simulation and electromagnetic analysis of the mask/EUV interactions that using a reduced bandwidth will lead to the same conclusions about printability as would be arrived at in an actual a stepper system.

\subsubsection{Zone placement error, contrast loss and flare}

The effect of zone placement error on imaging performance with a zone plate has been studied in the literature ${ }^{21}$. Two types of zone placement error were studied: random variations in zone placement, for example jitter in the electron beam, and calibration errors corresponding to stage drift. It was found that the far-field intensity pattern from a zone plate was a sensitive indicator of small zone positioning errors, and that this zone placement error was sufficient to degrade the optical performance. Random errors in zone placement had a similar effect to high spatial frequency errors, reducing contrast and diffraction efficiency, whilst abrupt errors caused during stage recalibration to compensate for drift displayed an effect similar to mid spatial frequency errors associated with flare. Comparing measured data from several manufactured zone plates with $80 \mathrm{~nm}$ outer zone showed image degradation consistent with $30 \mathrm{~nm}$ zone placement errors. This study dates from 1996, however, and there has been progress in zone plate fabrication technology since this date, thus it would be worthwhile repeating this study for current state-of-the-art zone plates as a part of technology qualification.

In the context of AIM imaging, the zone plate design for on-axis imaging has outer zones of $40 \mathrm{~nm}$ width, thus we would expect the effects of zone placement error on image quality to be somewhat more apparent than for a zone plate with $80 \mathrm{~nm}$ outer zone width. We note that $40 \mathrm{~nm}$ outer zone width is well within the resolution limit of current

${ }^{21}$ E.Tejnil, K.A.Goldberg, E.H.Anderson and J.Bokor "Zonal placement errors in zone plate lenses" OSA Trends in Optics and Photonics Vol. 4, Extreme Ultraviolet Lithography, G. D. Kubiak and D. R. Kania, eds. (Optical Society of America, Washington, DC 1996), pp. 138-142 
state-of-the-art zone plate fabrication techniques - for example the CXRO Nanowriter can produce feature sizes down to $4 \mathrm{~nm}$ in very slow resist (calixerene). However, based on studies in the literature we note that the accuracy of zone placement over the entire zone plate as well as smallest write-able feature size must be considered in determining the fabrication requirements of a zone plate for EUV mask inspection.

\subsubsection{Field curvature and off-axis astigmatism}

In addition to working distance and bandwidth there are several issues associated with using a single optical element which must be addressed in a single-element zone plate design. With only one optical element it is possible to obtain axial stigmatism, but it is not in general possible to design a single element lens that is astigmatic across the field of view. We therefore anticipate that a zone plate system, whilst stigmatic on axis, will in general suffer from astigmatism at off-axis field points. Astigmatism displays asymmetric behaviour through focus, thus the effects of offaxis astigmatism on the fidelity of through-focus image series needs to be carefully considered in an AIM application.

Furthermore, a single-element lens will in general suffer from curvature of field as the Petzval sum can not be driven to zero for a single element ${ }^{22}$, thus the plane mask surface will be imaged onto a curved image surface in the detector plane. Since both the mask and commercially available CCD detectors are inherently flat ${ }^{23}$ this curvature of field will lead to a change in focus across the field; depending on where in the field a feature is located it will be at a different defocus, which will in turn affect the CD measurement through focus. The effects of both astigmatism and field curvature increase towards the edge of the field, thus the effects of field curvature and off-axis astigmatism on aerial image need to be considered in a zone plate design when determining the useable field of view over which stepper-equivalent AIM measurements should be made.

\subsubsection{Preliminary throughput budget}

We now proceed to calculate a preliminary throughput budget for a zone plate system. As previously noted, the figures in this throughput budget represent the best estimate based on information to hand at the time of writing and keep as many parameters as possble consistent with previous calculations for the purposes of comparison with other implementations. This throughput budget is calculated for a CCD camera with 2048x2048, 13.5 micron pixels although we note that it is open to a vendor to select another CCD if desired.

In order to determine the required source power, and to ensure consistency between different approaches, we aim for a noise level of $1 \%$ in the detected image. In this regard it is important to point out that the source powers here are indicative only and it may well be feasible to use a smaller source, although exposure times will have to be lengthened, a number of frames integrated, or the allowed noise level increased to compensate for the lower EUV flux. In the interests of consistency we use the same values of common figures such as multilayer reflectivity as used in evaluating other systems so as to enable a fair side-by-side comparison of the various approaches.

\begin{tabular}{|lc|}
\hline Zone plate system & \\
\hline Preliminary throughput budget & Value \\
Target values & $1 \%$ \\
Noise level & $1 \mathrm{sec}$ \\
Exposure time & EEV $36 / 40$ \\
EUV CCD & $1.15 \%$ \\
Total system noise (total shot noise) & $13.5 \mathrm{um}$ \\
Pixel size & 100,000 \\
Electrons per full well & 100,000 \\
Measured number of electrons & 3.65 \\
eV/photon & 27.40 \\
electrons/photon & 3650 \\
photons per full well & 0.45 \\
Quantum efficiency (QE) & 8111 \\
Photons incident on a pixel & $811,111 \mathrm{eV}$ \\
Photon energy incident on a pixel & \\
& $2048 \times 2048$ \\
\hline Number of pixels (array) & \\
\hline
\end{tabular}

\footnotetext{
${ }^{22}$ Born and Wolf, Principles of Optics, $7^{\text {th }}$ ed., $§ 5.5 .3$.

${ }^{23}$ We note that curved detectors have been manufactured for custom applications, and are typically based on fibre-coupled CCDs which use the optical fibre bundle to transfer the curved detector surface to the CCD plane.
} 


\begin{tabular}{|c|c|}
\hline Number of pixels (total) & $4,194,304$ \\
\hline Energy incident on CCD & $3.40 \mathrm{E}+12 \mathrm{eV}$ \\
\hline EUV energy incident on whole CCD for full exposure & $0.54 \mathrm{uJ}$ \\
\hline \multicolumn{2}{|l|}{ Zone plate } \\
\hline Magnification & $500 x$ \\
\hline Collected NA & 0.0625 \\
\hline Nominal resolution & $107 \mathrm{~nm}$ \\
\hline Zone plate efficiency & $20 \%$ \\
\hline Membrane transmission & $50 \%$ \\
\hline Microscope throughput & $10 \%$ \\
\hline \multicolumn{2}{|l|}{ Mask } \\
\hline CCD pixel size at mask & $27 \mathrm{~nm}$ \\
\hline Pixels per resolution element (sampling) & 4.0 \\
\hline Field of view (square) & $55.3 \mathrm{um}$ \\
\hline Reflectivity of mask & $65 \%$ \\
\hline Wavelength matching factor & $98 \%$ \\
\hline Mask throughput & $64 \%$ \\
\hline Energy incident on mask per shot (over field) & $8.55 \mathrm{uJ}$ \\
\hline \multicolumn{2}{|l|}{ System loss factors } \\
\hline Spectral purity filter throughput & $35 \%$ \\
\hline Transmission through gas & $50 \%$ \\
\hline Transmission through debris shield & $80 \%$ \\
\hline Throughput contribution of system loss factors & $14 \%$ \\
\hline \multicolumn{2}{|l|}{ Condenser } \\
\hline Coherence (sigma) & 0.8 \\
\hline Illuminating NA & 0.05 \\
\hline Mirror reflectivity - normal incidence & $70 \%$ \\
\hline Mirror reflectivity - 45 degree & $32 \%$ \\
\hline Mirror reflectivity - grazing incidence & $90 \%$ \\
\hline Number of normal incidence reflections & 1 \\
\hline Number of 45 degree reflections & 0 \\
\hline Number of grazing incidence reflections & 3 \\
\hline Wavelength matching factor & $98 \%$ \\
\hline Condenser throughput & $51 \%$ \\
\hline Total optical system throughput & $0.46 \%$ \\
\hline Overfill factor (ratio of area) & 2 \\
\hline Illuminated area & $6115 u m^{\wedge} 2$ \\
\hline In-band EUV energy required into condenser per exposure & $239.22 \mathrm{uJ}$ \\
\hline
\end{tabular}

Table 14

Throughput budget for a zone plate system to achieve $1 \%$ noise level in the measured image.

Using the étendue arguments described in section 4 above we now proceed to calculate the minimum power requirements for two representative source sizes. Recall that étendue calculations assume a perfect loss-free optical system, thus the calculations below are based on the assumption of ideal throughput. In reality the condenser throughput may be less than ideal due, for example, to ray losses in the condenser system (the contribution due to reflection from 2 condenser mirrors has already been taken into account). 


\begin{tabular}{|lrc|}
\hline In-band EUV energy required into condenser per exposure & $\mathbf{2 3 9 . 2 2} \mathbf{u J}$ \\
Sources & 200 & $20 \mathrm{um}$ \\
Source size (diameter) & & $314 \mathrm{um}^{\wedge} 2$ \\
& & 0.221 \\
Source area & 31410 & $12.75 \mathrm{deg}$ \\
Collectable NA (etendue limited) & 0.022 & $2.5 \%$ \\
Collection angle (etendue limited) & 1.26 & $\mathbf{9 . 7 1} \mathbf{~ m J}$ \\
Fraction of 2Pi collected (etendue limited) & $0.0243 \%$ & $\mathbf{9 8 3}$ \\
Minimum In-band EUV energy required into 2Pi per exposure & 1.00 & $\mathbf{0 . 0 1} \mathbf{W a t t}$ \\
Exposure time & $\mathbf{0 . 9 8}$ & $\mathbf{1 . 9 4} \mathbf{W a t t}$ \\
Required in-band EUV power into 2Pi during exposure & $0.50 \%$ & $\mathbf{1 9 7}$ \\
Conversion efficiency & & \\
Driver power during exposure & & \\
\hline
\end{tabular}

Table 15

Source power requirements for a zone plate system to achieve $1 \%$ noise level in a $1 \mathrm{~s}$ exposure.

Such source powers are not unreasonable for a compact EUV source, although we once again note the strong motivation to use a small source for the AIM tool due to étendue limitations placed on collectable power. This power level is driven by the specification of detector $1 \%$ noise over a $1 \mathrm{sec}$ exposure and can be relaxed if the desired noise level is also relaxed, as illustrated in the box below:

\begin{tabular}{|l|l|l|l|}
\hline $200 \mu \mathrm{m}$ diameter source & $1 \%$ noise & $2 \%$ noise & $3 \%$ noise \\
\hline $1 \mathrm{~s}$ exposure & $0.98 \mathrm{~W}$ & $0.34 \mathrm{~W}$ & $0.15 \mathrm{~W}$ \\
\hline $10 \mathrm{~s}$ exposure & $98 \mathrm{~mW}$ & $34 \mathrm{~mW}$ & $15 \mathrm{~mW}$ \\
\hline
\end{tabular}

Table 16

In-band EUV power into $2 \pi$ per exposure for a $200 \mu \mathrm{m}$ diameter source to achieve a desired noise level in the final image (data taken from above spreadsheet). The $10 \mathrm{~s}$ exposure time is included for illustration-ideally the exposure time should be $<1 \mathrm{sec}$ for a practical system and must be negotiated between vendor and client.

It is therefore important to recognise that the power requirements listed above are part of a cost-benefit tradeoff between exposure time, noise levels, and source availability, and that source power requirements increase significantly as the target noise level is reduced. Furthermore, as noise levels can be reduced by increasing integration time, it may be desirable to design a two-mode system with a fast, higher noise scanning mode for navigation and a slower, low-noise mode for collecting AIM data. Rebinning the data so that four pixels are reduced to one pixel provides another avenue for decreasing noise and/or source power at the expense of spatial resolution and may be attractive for use in navigation mode. Such tradeoffs are a necessary part of the design process and will have to be optimised by each vendor individually.

The effect of bandwidth requirements for a zone plate system must also be considered. A zone plate system requires a significantly smaller bandwidth than a comparable system using normal incidence mirrors. As described in the preceding discussion, to achieve a practical working distance of $0.5 \mathrm{~mm}$ with the preferred on-axis imaging design (to avoid keystone distortion) it is necessary to reduce the bandwidth to $0.1 \%$. For a white-light source this reduced bandwidth comes at the expense of throughput because photons must be discarded in order to narrow the bandwidth, increasing the source power requirements. However we also note that there may be little penalty in terms of throughput if a source is chosen which has a strong emission line at $13.5 \mathrm{~nm}$ with sufficiently narrow intrinsic bandwith. In either case the effect of reducing bandwidth must be factored into the throughput budget in order to determine required source power.

\subsubsection{Technical risks and key comptetencies required of vendor}

Technical aspects of zone plate systems are well understood, and it appears that these components can be either sourced from vendors or manufactured as needed for integration into an AIM microscope. The main areas of technical risk appear to be the effect of bandwidth narrowing on the equivalence of aerial image performance to that of a scanner, and the optical performance of the zone plate, in particular with respect to flare, contrast and field curvature. 
In addition to the general system competencies required for system integration the vendor should demonstrate competency in the following areas relevant to this design approach:

- An optical design which emulates the salient features of a 6-mirror optical system, including flare and throughfocus behaviour.

- Demonstration of the contrast, flare and field of view of the proposed zone plate, taking into account manufacturing error, and analysis of how this will affect the fidelity of the aerial image measurement

- Analysis of the effect of using other than a $2 \%$ band width, if this approach is adopted.

\subsubsection{Technology development or technology transfer requirements}

The required technologies for manufacturing a zone plate are commercially available at this point in time; the question is one of optical performance. Key areas of development would be to:

1. Verify the MTF, contrast and flare of the zone plate to be used.

2. Evaluate performance of the zone plate with regard to contrast and focus across the field of view so as to confirm that field curvature effects will be negligible across the proposed field of view.

3. Demonstrate that bandwidth narrowing, if used, will not affect the aerial image performance.

\subsubsection{Useful references}

1. E.Tejnil, K.A.Goldberg, E.H.Anderson and J.Bokor "Zonal placement errors in zone plate lenses" OSA Trends in Optics and Photonics Vol. 4, Extreme Ultraviolet Lithography, G. D. Kubiak and D. R. Kania, eds. (Optical Society of America, Washington, DC 1996), pp. 138-142

2. P.Naulleau, C.H.Cho, E.M.Gullikson and J.Bokor "Transmission phase gratings for EUV interferometry" (2000) 7 J.Synch.Rad. 405 


\section{Conclusions}

A number of potential configurations for an EUV AIM tool have been studied and technical aspects of the design approaches investigated. The requirements for AIM mode of emulating stepper quality imaging have been spelt out and strategies developed for assessing the suitability of different approaches to satisfying the technical requirements for AIM mode. A synopsis of the approaches considered, and conclusions reached, is presented in Table 17 below.

Preliminary designs of several system configurations have been performed, and for each approach potential technical risks have been identified; illumination and source requirements have been analysed for each implementation and may factor into selection of technical approach. It is important to note that identification of risk or required development does not disqualify a particular approach, rather it serves to raise an issue which must be addressed in any proposal to build a tool. 


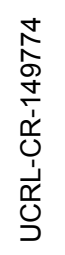

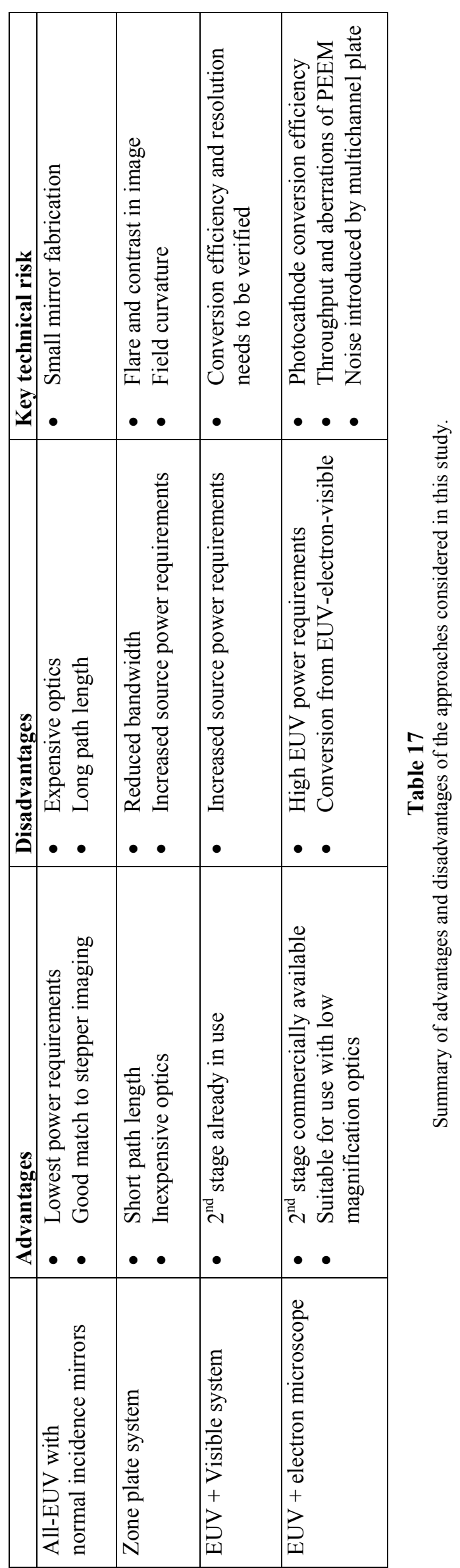

